\title{
Muscle: $50+$ Years of Electron Microscopy
}

\author{
Clara Franzini-Armstrong ${ }^{1}$ \\ 1 University of Pennsylvania
}

Funding: The author(s) received no specific funding for this work.

Potential competing interests: The author(s) declared that no potential competing interests exist.

\section{Abstract}

This project was initiated with the aim of preserving for the future a portion of the extensive muscle ultrastructural image archive accumulated over a 50 year period by the author and her laboratory. Between the late 1950s and current times electron microscopy (EM) evolved. The early EM revealed the ultrastructural identification and definition of cell organelles and laid the foundation for cell biology. This continues with he discovery of new structural organizations and organelles. Currently EM images provide the essential basis for identification of structural changes associated with mutational experiments and newly discovered pathology. The most recent development of techniques allowing near atomic level of resolution brings microscopy to modern times. This evolution has been greatly enriched by the rebirth of light microscopy, fueled by dynamic views of events based on video techniques, by the availability of specific targeting of cell components and by confocal microscopy that allows 3-D structural reconstructs. Correlation between light and electron microscopy, when appropriately done, is highly revealing.

The images in this publication present descriptive and structure-function correlations covering the whole animal kingdom with some areas more extensively illustrated than others. The first part classifies the images based in their site of origin, the second part identifies functional correlations, the third part introduces results of mutational experiments, the fourth part covers aging and pathology examples, the fifth part is a guide to the techniques. The images are presented with a numerical identification correlated with a very succinct description. References are limited to the directly relevant published material from the author's laboratory that are helpful in extending the limited legends and in assigning contributions. With very few exceptions, references to the literature are not given. The author asks for the forgiveness of the many friends, trainees, collaborators and competitors whose contributions and priorities are not recognized.

The work has been stimulated by interactions with teachers and advisors. Keith R. Porter, father of the endoplasmic reticulum and sarcoplasmic reticulum instilled admiration for Nature's work and was always been a guiding light in the author's work. Richard J. Podolsky and Andrew F. Huxley taught the importance of structural correlations. Past and present trainees and collaborators, whose names appear in the reference list, have added depth of understanding. In the case of skeletal muscle much necessary information is gained by using the muscle bible: "Myology", A.G. Engel and C. Franzini-Armstrong, Eds. 3rd Edition, McGraw Hill, N.Y. 2004.

\section{INDEX}




\section{Part 1 By Zoological and Tissue Classification}

1.1 Lower Invertebrates

1.1.1 Cnidaria, Hydrozoa

1.1.2 Worms

1.1.2.1 C. Elegans

1.1.2.2 Earthworm

1.1.2.3 Leaches

1.2 Molluscs

1.2.1 Bivalves

1.2.2 Cephalopods

1.3 Arthropods

1.3.1 Insects

1.3.1.1 Dragonfly DFM

1.3.1.2 Drosophila IFM

1.3.1.3 Blow Fly Body Muscles

1.3.2 Crustacea

1.3.2.1 Copepods

1.3.2.2 Crab

1.3.2.3 Crayfish

1.3.3 Arachnida

1.3.4 Polchidae

\subsection{Chordates}

1.4.1 Cephalochordates, Amphioxus

1.4.2 Primitive Fish

1.4.2.1 Garfish

1.4.2.2 Hagfish

1.4.2.3 Lamprey

1.4.3 Advanced Fish

1.4.3.1 Zebrafish

1.4.3.1.1 Zebrafish Gills

1.4.3.1.2 Zebrafish Intestine, Liver and Pancreas

1.4.3.1.3 Zebrafish Kidney

1.4.3.1.4 Zebrafish Tail Muscle

1.4.3.2 Toadfish

1.4.3.3 Midshipman

1.4.3.4 Sand Dab 
1.4.3.5 Glass Fish

1.4.3.6 Sea Horse

1.4.3.7 Goldfish

1.4.4 Reptiles

1.4.4.1 Turtle

1.4.4.2 Lizard

1.4.4.3 Snakes

1.4.5 Amphibia

1.4.5.1 Frog Twitch Fibers

1.4.5.2 Frog Tonic Fibers

1.4.6 Birds

1.4.7 Mammals

1.4.7.1 Cat Extraocular Muscles

1.4.7.2 Guinea Pig Leg Muscles

1.4.7.3 Mouse Fast Muscles

1.4.7.4 Mouse Slow Muscles

1.4.7.5 Rat Sternomastoid

1.4.8 Vertebrate Cardiac Muscles

1.4.8.1 Purkinje Bundle

1.4.8.2 Bird heart

1.4.8.2.1 Chick Corbular jSR

1.4.8.2.2 Finch PCs and EJSR

1.4.8.3 Frog Heart

1.4.8.4 Lizard Heart

1.4.8.5 Rat Heart

1.4.8.6 Mouse Heart

1.4.8.7 Rabbit and Dog Heart

1.4.9 Rat Cerebellum

1.4.10 Smooth Muscle

1.4.11 In vitro

1.5 Differentiation

1.5.1 $\mathrm{T}$ tubules

1.5.2 Triad Positioning

1.5.3 Triad Maturation

1.5.4 Myotubes and Myofibrils 


\section{Part 2 Structure-Function}

\subsection{The Contractile Machinery}

2.1.1 Myosins

2.1.2 Filaments and Cross Bridges

2.1.2.1 Myosin Filaments

2.1.2.2 Actin Filaments

2.1.2.3 Rigor Cross Bridges

2.1.2.4 Active Cross Bridges

2.1.3 Skeletal Muscle Sarcomeres

2.1.3.1 Sarcomere Bands

2.1.3.2 Thin Filament Lengths

2.1.3.3 Band patterns/Sarcomere Lengths

2.1.3.4 Section Thickness Effects

2.1.3.5 Thin/Thick Filaments

2.1.4 Myofibrils Cross Striation

2.1.4.1 Myofibrils

2.1.4.2 Cross striation, Vernier

2.1.5 Arthropod Muscles, Myofibrils and Sarcomeres

2.1.5.1 Sarcomere Bands

2.1.5.2 Myofibrils

2.1.5.3 Thin/Thick Filaments

2.1.6 Lower Invertebrate Muscles

2.2 Structural Cross Linkers, Z and M lines

2.2.1 Simple Vertebrate $Z$ Lines

2.2.2 Complex Vertebrate Z Lines

2.2.3 Invertebrate Z Lines

2.2.4 M Line (band)

2.3 SR and the Calcium Cycle

2.3.1 SR Structure

2.3.1.1 Segmented SR in Skeletal Muscle

2.3.1.2 Continuous SR in Cardiac and Invertebrate Muscle

2.3.2 SR as a $\mathrm{Ca}^{2+}$ Store

2.3.3 SR ATPase

2.3.4 SR as a Calcium Source

\subsection{The T Tubules}

2.4.1 T Tubule Openings 
2.4.2 Skeletal Muscle Transverse T Networks

2.4.3 Skeletal Muscle Longitudinal T Extensions

2.4.4 Junctional and Free T Tubule Segments

2.4.5 Membrane Folds, jT, fT in Arthropod Muscles

\subsection{E-C Coupling CRUs}

2.5.1 Variety of CRUs

2.5.1.1 Triads

2.5.1.2 Dyads

2.5.1.3 Peripheral Couplings (PCs)

2.5.1.4 Corbular and EJSR

2.5.2 Structural Components of CRUs

2.5.2.1 Feet and RyR

2.5.2.2 Tetrads and DHPRs

2.5.2.3 Calsequestrin

2.5.2.3.1 Calsequestrin Identification

2.5.2.3.2 Structural Components of CRUs

2.5.2.3.3 Polymer Structure of Calsequestrin

2.5.2.4 Stac3

2.5.2.5 DHPR in Cardiac and Invertebrate Muscles

2.5.3 Isoform-Specific Molecular Arrangements

2.5.3.1 RyR1 and CaV1.1 in Skeletal Muscles

2.5.3.2 RyR2 and CaV1.2 Cardiac Muscle

2.5.3.3 RyR3 and CaV1.2 Equivalent in Invertebrates

2.5.3.4 RyR3 in Skeletal Muscle

2.5.3.5 Models

\subsection{Other CRU Components}

2.6.1 Immunostaining of CRU Components

2.6.2 Visualization of Junctin and Triadin

\subsection{Fiber Types}

2.7.1 Tonic and Twitch Fibers in Skeletal Muscle

2.7.2 Variety of Twitch Fibers

2.7.2.1 Glycogen

2.7.2.2 Mitochondria and Lipids

2.7.2.3 Golgi "Stain", JT and jSR

2.7.2.4 Z Line Width

2.7.3 Insect Flight Muscle Dichotomy - DFM and IFM 


\subsection{Mitochondria}

\subsubsection{Cristae}

2.8.2 Continuities

2.8.3 Distribution

2.8.4 Positioning

2.8.5 Kissing Junctions and Nano Tunnels

2.8.6 Dynamics

\subsection{IP3Rs}

2.9.1 IP3Rs in Nuclear Envelope

2.9.2 IP3Rs and RyRs: Calcium Microdomains

2.9.3 IP3Rs in ER Stacks

\subsection{SOCE}

2.10.1 STIM/ORAI System

2.10.2 Calcium Entry Units in Skeletal Muscle

\subsection{Differentiation of the Sarcotubular System}

2.11.1 Birth of CRUs

2.11.2 CRU Positioning

2.11.3 T Tubules and Triads

2.11.4 Maturation

2.12 Cell Junctions

\subsection{Other Organelles}

2.13.1 Caveolae, Aquaporins and Lysosomes

2.13.2 Microtubules, Golgi System, Nuclei

2.14 Muscle Tissue, Accessory Components

2.14.1 Capillaries and Pericytes

2.14.2 Satellite Cells, Fibroblasts

2.14.3 Myotendon Junction, Spindle

2.14.4 Axons and Nerves

2.14.5 Neuromuscular Junction

\section{Part 3 Experiments}

3.1 Exploring RyR, DHPR and Stac3 Roles

3.1.1 DHPR in Dysgenic Muscle

3.1.2 DHPR Tetrad Rescue

3.1.3 Dyspedic Mutation and Rescue

3.1.4 Roles of RyR1 and RyR3 
3.1.4.1 In vitro Experiments

3.1.4.2 Sparks

3.1.4.3 Crooked Neck Dwarf Chicken

3.1.5 Role of DHPR $\beta$ Subunit

3.1.6 RyR-DHPR Double Knockout

3.1.7 Stac 3

3.2 Null Mutations and Overexpression

3.2.1 Cardiac CRU Proteins Overexpression

3.2.2 Cardiac Junctophilin Overexpression

3.2.3 Engineered Mutations

3.2.3.1 Skeletal CASQ Null

3.2.3.2 Cardiac CASQ Null

3.2.4 SR ATPase Reduction

3.2.5 Cardiac Triadin and Junctin Null Mutations

3.3 Expression in CHO Cells

3.4 Myosin Exchange in Drosophila Muscle

Part 4 Age and Pathology

4.1 Age Related Alterations

4.1.1 CASQ and Tubular Aggregates

4.1.2 Vernier Displacements and Z Line Streaming

4.1.3 Mitochondria Redistribution

4.2 Mouse Models of Malignant Hyperthermia

4.2.1 RyR1 Y522S Mutation, Central Core

4.2.2 RyR1 ${ }^{\mathrm{T} 486 \mathrm{I}}$ Mutation

4.2.3 RyR1 ${ }^{\text {I4895T }}$ Mutation

4.3 Modeling Cardiac Mutations

4.3.1 Cardiac CASQ ${ }^{\mathrm{D} 307 \mathrm{H}}$ Mutation

4.3.2 Mitochondria-Related Mutation

4.4 Zebrafish Twister

\section{Part 5 Techniques}

5.1 Standard Techniques for Thin Sections

5.1.1 Preparing Tissue

5.1.1.1 Dissection 
5.1.1.2 Fixation in situ

5.1.1.3 Fixation by Perfusion

5.1.1.4 Biopsies

5.1.2 Primary Chemical Fixation

5.1.3 Contrast Enhancement

5.1.4 Dehydration

5.1.5 Embedding

5.1.5.1 Organs and Tissues

5.1.5.2 Isolated Cells and Organelles

5.1.6 Thin Sectioning

5.1.6.1 Sectioning and Staining

5.1.6.2 Collecting sections

5.1.6.3 Section "Staining"

5.2 Additional Techniques

5.2.1 T Tubule Labeling

\subsubsection{Skinning}

5.2.3 Fluorescent Membrane Dyes

\subsection{Imaging Small Objects}

5.3.1 Negative Staining

5.3.2 Rotary Shadowing

\subsection{Cryotechniques}

5.4.1 Freeze-Fracture and Deep Etch

5.4.2 Rapid Freezing

5.4.2.1 Catching Rapid Events

5.4.2.2 Pressure Freezing for EM Tomography

\subsection{SEM Techniques}

5.5.1 Exposing Cell Surfaces

5.5.2 Exposing Internal Structures

\subsection{Immunolabeling}

\subsection{Histochemistry of Motor End Plates}

\section{Part 1 By Zoological and Tissue Classification}

1.1 Lower Invertebrates

1.1.1 Cnidaria, Hydrozoa

Cnidaria are at the stem of the metazoan evolutionary tree. They are the first animals with tissue layers, but they are diplobastic (two layers ecto- and endo-derm), although they have some primitive muscles (1.1.1 A). The cnidaria have 
some primitive $Z$ lines that are similar to those in smooth muscles of vertebrates.

Hydra is a genus of small fresh-water organisms in temperate and tropical regions. It has tubular body with tentacles at mouth opening. Two tissue layers: epidermis and gastrodermis are separated by a gelly of mesoglea. Body and tentacles can retract due to muscle contraction $(1.1 .1 \mathrm{~B}, \mathrm{C})$

\subsubsection{Worms}

\subsubsection{C. Elegans}

Caenorhabditis elegans is a free-living, transparent nematode (round worm), about $1 \mathrm{~mm}$ in length, that lives in temperate soil environments. Over 50 years ago, Sydney Brenner established it as a genetic model for understanding questions of developmental biology and neurobiology. A single layer of muscle cells, partially divided into fibers, is immediately under the cuticle. Mitochondria occupy the deeper layer of the cells (1.1.2.1 A). The striation is oblique so in transverse sections all bands are shown. Thin/thick filament ratio is high and variable (1.1.2.1B). Peripheral couplings are present in the membranes of clefts delimiting the muscle fibers are (1.1.2.1 C). Some membrane invaginations are equivalent to primitive T tubules (1.1.2.1 D). Hong et al., 2001; Burkeen et al., 2004.

\subsubsection{Earthworm}

Earthworms are annelids, based on appearance of surface rings. They live on discarded vegetation, digesting it and producing top soil. Most of the body is a solid mass of rapidly moving oblique striated muscle. In cross sections the fibers' outlines are elongated cylinders. T tubules are absent, the small myofibrils alternate with bands of SR (1.1.2.2 A, B). In longitudinal sections long thick filaments compose thin myofibrils and are attached via the thin filaments to dense bodies equivalent to $Z$ lines. SR elements are intercalated between the dense bodies (1.1.2.2 C). In other thin sections orientations the oblique sarcomeric arrangement and the abundant SR elements at the $Z$ line level are clearly visible (1.1.2.2 D, E). Elongated SR elements at the $Z$ line connect to large PC cisternae devoid of calsequestrin (1.1.2.2 F).

\subsubsection{Leaches}

Leeches are segmented parasitic or predatory worms that belong to the phylum Annelida and comprise the subclass Hirudinea. They are closely related to the oligochaetes, which include the earthworms, and like them have soft, muscular, segmented bodies that can lengthen and contract extensively The muscle are oblique striated, with very long, thick myosin/paramyosin filaments of large diameter (1.1.2.3 A-C). Both actin/myosin sliding and rearrangement of the thick filaments allow extreme length changes.

\subsection{Molluscs}

\subsubsection{Bivalves}

Bivalve molluscs (e.g., clams, oysters, mussels, scallops) have an external covering that is a two-part hinged shell containing the soft-bodied organism. Scallop muscles have been the subject of classical studies of the calcium control of myosin ATPase. The muscle contains two adjacent but quite distinct muscle bundles. The fast muscle is responsible for phasic valve closing in swimming, the slow muscles for keeping the valves shut.

Thew fast muscle is composed of fibers containing single myofibrils. SR and glycogen reside in a narrow space between plasmalemma and myofibrils (1.2.1 A). The muscle is cross striated, an unusual structure for a mollusc (1.2.1 B). The A 
bands have a well defined, central, bridge-free region, but no $\mathrm{M}$ line. The $\mathrm{Z}$ lines are wavy, the edges pf the $\mathrm{A}$ band are not well defined and the length of $A$ band is somewhat variable. A full complement of 12 thin filaments surrounds the thick filaments (1.2.1 B). An occasional Vernier mismatch (see. 2.1.4.2) may also be present. An extensive free SR occupies the space under the plasmalemma, and PC with visible feet are present (1.2.1 C). The free SR has an unusual crystalline arrangement of calcium ATPase (1.2.1 D). PC cisternae are apparently empty, so the content of CASQ is either low or null. DHPR particles in freeze-fractures of the plasmalemma are NOT arranged into tetrads (1.2.1 E).

The slow muscle has no cross striation, but long paramyosin/myosin filaments associated with variable numbers of thin filaments and frequent PCs with empty SR cisternae. Adhesion sites are at the fiber edge (1.2.1 F, G). Nunzi and FranziniArmstrong, 1981, Castellani et al., 1989.

\subsubsection{Cephalopods}

Cephalopods are the most intelligent, most mobile, and the largest of all molluscs. They include squid, octopuses, cuttlefish, the chambered nautilus, and their relatives. These exclusively marine animals are characterized by bilateral body symmetry, a prominent head, and a set of arms or tentacles modified from the primitive molluscan foot. The squid propels itself by a strong coordinated contraction of the mantle that forces water backwards.

Squid muscle is illustrated. The myofibrils form a peripheral ring surrounding an area rich in mitochondria (1.2.2 A). The striation is "oblique" and a variety of appearances are created in longitudinal sections (1.2.2 B). In sections parallel to the thin/thick filaments, the very narrow I band/Z line segments are at an oblique angle to the thick filaments in the $A$ band (1.2.2 C). The thin filaments gather into small dense regions (equivalent to $Z$ lines), alternate with $S R$ elements (1.2.2 $D, E)$. T tubules are absent and the dilated jSR cisternae, apparently empty of calsequestrin, form PCs at the fibers' edges (1.2.2 F, see also $\mathrm{H}$ ) with no specific relation to the striation pattern.

\subsection{Arthropods}

Arthropods have a chitinous exoskeleton. . Muscle fibers are cross-striated, with a classical disposition of sarcomeres with thin and thick filaments and $Z$ lines, but no $\mathrm{M}$ lines. The length of thin and thick filaments and the thin/tick filament ratios vary across the species and depending on the fibers' rate of contraction so that the resting sarcomere lengths varies over a wide range.

\subsubsection{Insects}

Insects have a three-part body (head, thorax and abdomen), three pairs of jointed legs and 1-2 pairs of wings, compound eyes and one pair of antennae. Insects are the most diverse group of animals; they include more than a million described species and represent more than half of all known living organisms.

As in other arthropoda, the ends of muscle fibers are attached to a thin extracellular matrix that mediates direct connection to the chitin exoskeleton. In some muscles a dense flat fascia in the center of the muscle acts as tendon, allowing an additional central attachment of muscle fibers. Muscle fibers are cross-striated, with a classical disposition of sarcomeres with thin and thick filaments and $\mathrm{Z}$ lines, but no $\mathrm{M}$ lines. The length of thin and thick filaments and the thin/tick filament ratios vary across the species and depending on the fibers' rate of contraction so that the resting sarcomere lengths varies over a wide range.

The major muscle mass constitutes the flight muscle, in the thorax. The flight muscles have two basic contraction 
mechanisms. In the direct flight muscles a nerve signal initiates a contraction followed by relaxation. The muscle directly pulls the wing down when it contracts. Flight muscles in dragonflies and mayflies as well as body muscles in all insects use the direct mechanism. In the indirect flight mechanism the muscles invest their energy into deforming the insect's thorax, and the energy stored is released causing the wings to move up and down in turn. A single nerve impulse raises the cytoplasmic calcium which remains high for a fairly prolonged period of time during which the wings beat at high frequency in response to stretch activation. The majority of flying insects have indirect flight muscles, including butterflies and moths, beetles, grasshoppers and crickets, flies and bees.

\subsubsection{Dragonfly DFM}

The direct flight muscle of dragonflies sustains movement for long periods of flight and it is so fast that that the wings in flight appear blurred. Adaptations for this performance include: very thin ribbon-like myofibrils (1.3.1.1 A, B); large mass of mitochondria and a very frequent and highly organized close association of SR, T tubules with the mitochondria (1.3.1.1C, D); highly convoluted free SR and extensive ordered plaques of RyRs at CRUs (1.3.1.1 E). CRUs are located at frequent intervals in groves of the mitochondria surface, with the T tubule membrane closely apposed to the mitochondrial surface (1.3.1.1 F). One side of the $T$ tubule faces the $j S R$ and cytoplasmic leaflet contains clusters of DHPRs, NOT organized into tetrads (1.3.1.1 G, H). Takekura and Franzini-Armstrong, 2002.

\subsubsection{Drosophila IFM}

Drosophila, fight muscles are indirect (see 2.1.5.20). IFMs are called fibrillar because the small fibrils with a circular cross section are separated from each other by large spaces, mostly containing mitochondria (1.3.1.2 A, B). This makes the myofibrillar structure very evident at the light microscope level. Trabeculae carrying air tubes penetrate into the muscle (1.3.1.2 B). The most striking structural features are the large size of mitochondria well endowed with cristae, the frequent CRUs (dyads) and the scarcity of sarcoplasmic reticulum (1.3.1.2 C, D). These features are consistent with the physiology of a muscle that needs to be activated fast but does not reduce the free calcium concentration rapidly and that uses energy for the flight. IFMS have a most regular crystalline arrangement if thin and thick filaments (1.3.1.2 E), see 2.1.2.4 folder.

\subsubsection{Blow Fly Body Muscles}

This group of images compares two body muscles in Calliphora (a large fly). The flight control muscle acts on wing positioning during flight and thus it must be fast acting. In keeping with this function the fibers have small myofibrils and an abundance of CRUs and free SR (1.3.1.3 A and B). The TDT (tergal depressor of the trocanter) muscle powers the jump that immediately precedes the initiation of flight. It is fairly fast and powerful, but not geared for repetitive action. It has large myofibrils and a moderate content of CRUs and free SR (1.3.1.3 C). TDT muscles have unusual T tubules: Two rows of flat $\mathrm{jT}$ cisternae extend on either side of the tubules and form dyadic contacts with the jSR (1.3.1.3 D, E). The cytoplasmic domains of the jSR cisternae are decorated by numerous DHPR particles NOT clustered into tetrads (1.3.1.3 $F, G)$ and similar particles are located in junctional plaques of the plasmalemma at sites of $P C,(1.3 .1 .3 \mathrm{H})$. Takekura and Franzini-Armstrong, 2002.

\subsubsection{Crustacea}

\subsubsection{Copepods}


Copepods are small mesopelagic crustacea noticeable for the rapid movements of their appendages. Gaussia sp. is quite small and particularly fast. The arrangement of contractile material is unusual. Myofibrils, separated from each other by a thin layer containing $S R$ and $T$ tubules, are unusually large, but they are infiltrated by $T$ and $S R$ elements $(1.3 .2 .1 \mathrm{~A})$ thus rendering the frequency of $\mathrm{CRUS}$ and free SR appropriate for fast acting muscles. Both $\mathrm{T}$ and SR have frequent longitudinal components (1.3.2.1 B, C). CRUs, in the form of dyads, are elongated and longitudinally oriented, so that in cross sections they appear as $2-3$ small profiles (one $\mathrm{j} T$ tubule and 1 or $2 \mathrm{jSR}$ ) separated by feet (1.3.2.1 D). DHPRs are present along the elongated jT elements and they are frequent, but NOT arranged in tetrads (1.3.2.1 E). Muscles responsible for the movement of various appendage vary on their speed of contraction by up to three fold, but even the slowest ones are relatively fast and they all have the 3:1 thin/thick filament ratio typical of fast arthropod muscles (1.3.2.1 D). The A band length varies in relation to the speed, being shortest in the fastest fibers (compare 1.3.2.1 F, G, H, I, J) and see summary image (1.3.2.1 K). Even the longest sarcomeres are shorter than those in crab leg muscles (see 1.3.2.2 J). Glaser at al., 2018.

\subsubsection{Crab}

The fibers in the walking leg muscles of a large crab (Portunus sp.) have variable rates of shortening rates but overall within the range of relatively slow fibers when compared with copepods (see 1.3.2.1, copepods). Contrary to vertebrates, contraction rates do not depend on myosin isoform composition, but on innervation and sarcomeric structure. The muscle fibers are very large, but infiltrated by extensive folds of the surface membrane that effectively divide the fibers into smaller units (1.3.2.2 A, B ). T tubules invaginate from the folds and establish contact with the SR, forming dyads (1.3.2.2 C). The free SR is an extensive lacy network, longitudinally continuous. In proximity of T tubules the SR forms flat cisternae that are coupled to complementary jT cisternae within longitudinally oriented dyads (1.3.2.2 D, E). Folds and T tubule invaginations are visible in phase contrast light microscope images of Golgi stained muscle (1.3.2.2 F). The slower fibers have long sarcomeres and the tubular surface membrane invaginations are of two types. One set of invaginations, located at the $\mathrm{Z}$ line level, does not form associations with the SR, while the other, the functional T tubules at the A-I junction level, do (1.3.2.2 G). The two sets of T tubules were detected by Veratti in 1902 and are visible in light microscope images (1.3.2.2 H). The long sarcomere fibers have a double array of thin filaments with a 6:1 ratio to thick filament typical of slower fibers (1.3.2.2 I). Fibers of intermediate speed have a thin to thick filament ration less than 6.but not as low as 3:1. (not shown). A major characteristic is the variable length of $A$ bands, due to intercalated growth of sarcomeres that results in misalignment of the cross striation (1.3.2.2 J). Franzini-Armstrong, 1970, Franzini-Armstrong et al., 1986.

\subsubsection{Crayfish}

The abdominal flexor muscles occupy most of the tail in crawfish. The tail flip, engaged in the escape reaction, is very fast, but the muscle fatigues after very few contractions. The small muscle fibers have some membrane invaginations or folds, but smaller than in the crab leg muscles. Golgi staining shows an extraordinary abundance of T tubules arranged in a tight network without a preferential orientation. (1.3.2.3 A-C). Comparison of SR and T tubules in the same fibers show that the free $S R$ is relatively scarce but jSR cisternae, associated with $T$ tubules are frequent (1.3.2.3 D). T tubules are compared in light microscope and EM images (1.3.2.3 E). The abdominal flexor muscles are fast and so they have a filament disposition comparable to those of the insect flight muscles, with a 3:1 ratio of thin/thick filaments. The cross bridges in rigor have a regular distribution (1.3.2.3 H.J), see 2.1.2.3 D. Active zones along the actin filaments are even 
spaced and decorated by sets of cross brides coming from the two adjacent filaments (1.3.2.3 I).

Muscles in the appendages have longer sarcomere lengths and are quite similar to those in crabs (1.3.2.3 F, G, compare with 1.3.2.2 F). Eastwood et al., 1982.

\subsubsection{Arachnida}

Scorpions and spiders belong to the same class (arachnids). Their body muscles have many ultrastructural features in common. In both groups the openings of $\mathrm{T}$ tubules are patent and are in the form of slits (1.3.3 A, B scorpion, $\mathrm{C}$ tarantula spider). CRUs are in the form of dyads and often two dyads are present on two sides of each T tubule profile. The jSR portion associated with feet extends beyond the outlines of T tubules (1.3.3 D, scorpion; E spider). RyRs are arranged in large plaques on the jSR membrane with an orthogonal symmetry, similar to but not identical to that in skeletal muscle (1.3.3 F, G, H scorpion, J spider). The flat regions jT membrane that participate in the dyads are decorated by particles belonging to DHPR that are NOT organized into tetrads. (1.3.3 K scorpion; L, M spider). Crude muscle homogenates contain a mixture of actin and of very long myosin filaments with tapered ends and a bridge free region in the center. The helical arrangement of the relaxed cross bridges is well preserved in the isolated filaments (1.3.3 N-P), see 2.1.2.1 A, B. Franzini-Armstrong 1974, 1979, Takekura and Franzini-Armstrong, 2002.

\subsubsection{Polchidae}

"Daddy longlegs" are a family of araneomorph spiders first described by Ludwig Carl Christian Koch in 1850. Unlike true spiders, in which the body is divided into two distinct segments (the cephalothorax and the abdomen), daddy longlegs have only one segment. Legs are much longer than body and quite thin. The muscles are arranged in a tight spiral within the legs and attached to the surface chitin. The muscle fibers are similar to those of other crustacea, with large membrane folds that subdivide the fiber into small subunits (1.3.4 A, B). T tubules originate from the folds (1.3.4 B,C) and make dyadic contacts with jSR (1.3.4 D). The free SR is fairly abundant (1.3.4 E) and the sarcomeres are quite long, befitting fairly slow fibers (1.3.4 F).

\subsection{Chordates}

The transition between invertebrates and chordates is marked by gene duplications and a corresponding diversification of tissue and cell components. Prochordates are at the transition, they belong to the chordates phylum of animals having (at least at some stage of development) a notochord; dorsally situated central nervous system; and gill slits. They include vertebrates, tunicates and cephalochordates Chordates have an internal skeleton. Voluntary muscles are attached to the internal skeleton and thus they are called "skeletal muscles".

In the case of muscles the new gene products involve fiber type specific contractile and accessory proteins of the sarcomere, and the expression of two new isoforms: RyR1 in the SR and Cav1.1 in the T tubules. These are two major actors in e-c coupling that have the novel property of directly interacting with each other. The resulting e-c coupling step is not faster than the equivalent event in invertebrates, but it is more energy efficient, because it does not require calcium entry through CaV1. Indeed CaV1.1 of some fish muscles effectively interacts with RyR1, but it does not carry any current. Cardias muscle retains the archaic interaction between its isoforms (RyR2 and Cav1.2) requiring calcium to act as 
intermediate.

In order to interact with RyR1, four CaV1.1 must be associated with each RyR1 and this results in groups of four particles (tetrads) visible in freeze fracture images. The phylogeny tree below illustrates the appearance of tetrads in the early fish, but not in prochordata.

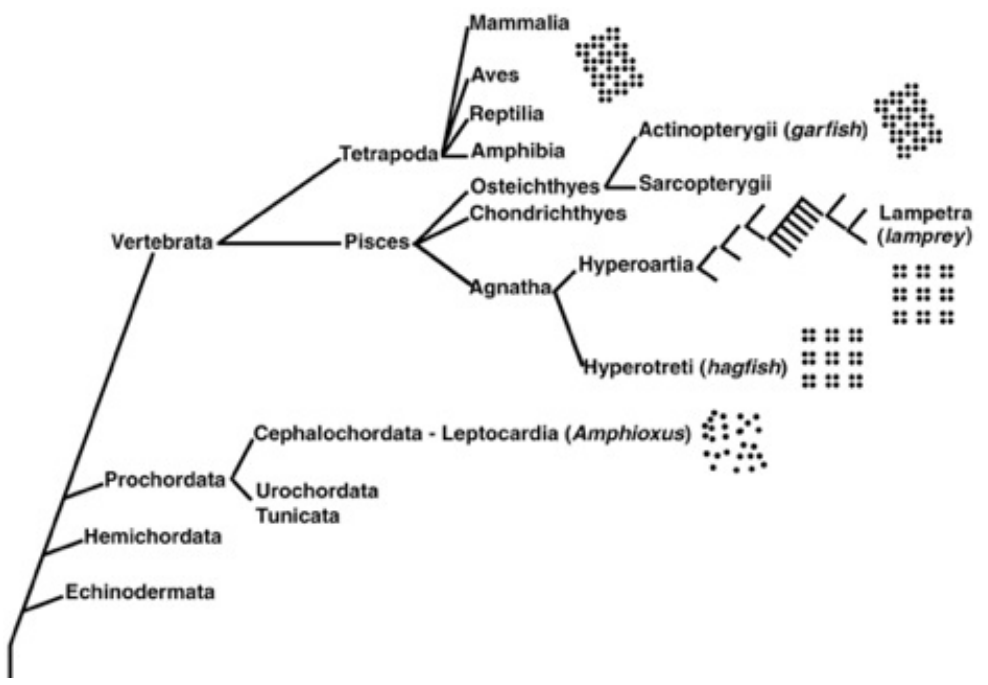

The dots represent DHPRs. Thefirst evolutionary appearance of DHPR tetrads, essential to skeletal muscle e-c coupling (see 2.5.3.1 folder) is in the lower vertebrates, represented by primitive fish.

Di Biase, and Franzini-Armstrong, 2005, Franzini-Armstrong, and Di Biase, 2007.

\subsubsection{Cephalochordates, Amphioxus}

Amphioxus, a primitive chordate, is at the transition between invertebrates and vertebrates. The muscle composing the body myotomes is quite unusual: the muscle fibers are very small (essentially composed of a single large myofibril), with a reverse innervation: the muscle cell reach the central nervous system rather than vice versa. No T tubules are necessary and the SR is located between the single myofibril and the edge of the fiber (1.4.1 A). A distinguishing feature is the frequency of dilated SR elements forming peripheral couplings at the cells' edges (1.4.1 B). In longitudinal sections. PC location is in proximity of the $\mathrm{Z}$ line and of the very short I band $(1.4 .1 \mathrm{C})$. The junctional SR cisternae at PCs are dilated and contain little if any calsequestrin, but feet are present (1.4.1 D). Freeze-fracture views of the plasmalemma show small mounds organized in rows of 2-3 elements at periodic intervals. These correspond to the sites where the dilated SR cisternae push into the plasmalemma, that is to PCs (1.4.1 E). The mounds are decorated by large particles with the structural signature of DHPRs $(1.4 .1 \mathrm{~F})$. Comparison of thin sections and freeze fracture images shows that while the feet are periodically disposed in the junctional gap the DHPR particles have no ordered arrangement and do not aggregate into tetrads (1.4.1.G). This indicates that the novel RyR-DHPR relationship of skeletal muscle is an evolutionary event that follows the appearance of the early chordates. Di Biase and Franzini-Armstrong 2005, Franzini-Armstrong and Di Biase, 2007.

\subsubsection{Primitive Fish}




\subsubsection{Garfish}

Garfish is an ancient bony fish. It has $T$ tubules at the $Z$ line level that subdivide the fibers into large myofibrils (1.4.2.1 A). The jSR cisternae are small, elongated longitudinally and associated with the T tubules and the plasmalemma to form frequent dyads and peripheral couplings (1.4.2.1 B). In both CRUs the junctional membranes have an unusual longitudinal axis $(1 \cdot 4.2 .1 \mathrm{C})$ and the $\mathrm{SR}$ cisternae are filled with a loosely packed calsequestrin polymer. The free $\mathrm{SR}$ is well developed, but the freeze-fracture particles representing the calcium ATPase protein are less frequent than in higher vertebrates (1.4.2.1 C). Peripheral coupling sites are located at transverse bands at the $Z$ line level (1.4.2.1 D). The DHPR particles in the junctional patches of plasmalemma membrane are arranged into tetrads (1.4.2.1 E), but with array parameters different from those of higher vertebrates, see 2.5.3.1 K. L. Garfish structure is a guide to vertebrate evolution. Di Biase and Franzini-Armstrong 2005, Franzini-Armstrong and Di Biase, 2007.

\subsubsection{Hagfish}

The hagfish is a primitive craniate considered to be either a transitional species between low chordates and vertebrates or the most primitive living vertebrate. It has T-SR triads remarkably similar to those of higher vertebrates, located at the A-I junction (1.4.2.2 A), some with a longitudinal orientation (1.4.2.2 B). Small peripheral couplings decorate the surface membrane (1.4.2.2 C). DHPR clusters at PC sites have clearly defined well arranged tetrads (1.4.2.2 D, E), but with array parameters different from those of higher vertebrates, see 2.5.3.1 K, L. Di Biase and Franzini-Armstrong 2005, FranziniArmstrong and Di Biase, 2007.

\subsubsection{Lamprey}

Lamprey is a cartilage fish distantly related to hagfish. It has frequent sometimes double triads at the A-I junction, with scarce calsequestrin (1.4.2.3 A). Triads and peripheral couplings coexist, but the latter are at the $Z$ line level (1.4.2.3 B,C). The SR is well developed, with a good complement of membrane particles denoting calcium ATPase (1.4.2.3 D). Plaques of DHPRs mark the location of elongated PCs at the the level of the $Z$ line (1.4.2.3 E). DHPRs are located in well developed tetrads., but with parameters different from those of higher vertebrates, see 2.5.3.1 K, L. Gap junctions are also present (1.4.2.3 F, G). Di Biase and Franzini-Armstrong 2005, Franzini-Armstrong and Di Biase, 2007.

\subsubsection{Advanced Fish}

\subsubsection{Zebrafish}

\subsection{Zebrafish Gills}

Fish gills are organs that allow exchange of oxygen and carbon dioxide between blood and water. They are located in gill slits on either side the pharynx, protected by a cover that allows water to flow over the feathery arches. The arches are composed of an internal capillary core covered on both sides by an epithelium that is in contact with the water (1.4.3.1.1 A, B). The epithelium s very thin allowing for rapid gas exchanges (1.4.3.1.1 C). Occasional larger, basically located cells are thought to be neuroepithelial and perhaps detect gas levels (1.4.3.1.1 D, E). Giuseppe Madeo, unpublished 1.4.3.1.2 Zebrafish Intestine, Liver and Pancreas

The images are from $\sim 7$ days PF zebrafish larvae. The epithelial cells of the large intestine have apical regions with tight junctions (1.4.3.1.2 A) and extensive microvilli with a halo of associated glycoproteins (1.4.3.1.2 B, C). The liver canaliculi at this stage are intra rather than inter-cellar (1.4.3.1.2 D). The space of Disse is extensive (1.4.3.1.2 E). Mitochondria are 
few but large (1.4.3.1.2 F) and ER cisternae are closely associated with the mitochondria (1.4.3.1.2 G). Glycogen is segregate within large areas of the cell (1.4.3.1.2 H). Exocrine pancreas has extensive RER associated with mitochondria (1.4.3.1.2 I, J), sometimes in an extensive swirl (1.4.3.1.2 K). Monk et al., 2013.

\subsection{Zebrafish Kidney}

The kidney in zebrafish has the same components as kidneys in higher vertebrates, but it is spread over a large distance in the body. Its structure is compared here with images from SEM of rat glomeruli. The glomerulus is a the tight ball of capillaries surrounded by podocytes and other accessory cells (1.4.3.1.3 A, rat). The endothelium of large convoluted capillaries is closely covered by feet processes of podocytes (1.4.3.1.3 B, C, fish). Each podocyte sends numerous long tentacles that associate with several capillaries, leaving small slits (1.4.3.1.3 D, rat). The filtration barrier between lumen of capillaries and the urinary space is in the slits between podocytes where access to the urinary space is through the combined basal laminae (1.4.3.1.3 E, F, fish). Mesangial cells of the glomerulus interstitium patrol the space removing debris (1.4.3.1.3 G fish). The proximal convoluted tubules are identified by the basal mitochondria and luminal cilia (1.4.3.1.3 $\mathrm{H}$, I fish). The apical side of the epithelium has long microvilli (1.4.3.1.3 J fish) and the basal side deep invaginations between the elongated mitochondria (1.4.3.1.3 K fish).

\subsection{Zebrafish Tail Muscle}

The myofibrils are flat and radially oriented as in other fish muscles (1.4.3.1.4 A, see 1.4.3.2 E). Even in very young larvae (72 hrs pf), muscle fibers in the most rostral sarcomeres have a totally differentiated structure with aligned myofibrils and frequent triads located at the $Z$ lines (1.4.3.1.4 B). Complete double (occasionally triple) arrays of feet accompany the junctional SR and $Z$ line structure is well established (1.4.3.1.4 C). In the younger fibers of caudal myotomes, the SR forms frequent PCs, which have orthogonal arrays of DHPR tetrads (1.4.3.1.4 D, E). Mitochondria are at the fiber surface, under the plasmalemma (1.4.3.1.4 F). See 2.5.3.4.

\subsubsection{Toadfish}

Muscle bands on the sides of the toadfish swimbladder belong to a category of super-fast fibers that produce vibration of the bladder wall and sounds by their contractions. Toadfish swimbladder muscles have been used for several basic studies and their structure reflects the fibers' properties.

Thin myofibrils and the sarcotubular complex occupy approximately equal volumes. Triads are two per sarcomere and the free $S R$ is very abundant (1.4.3.2 A). Double rows of feet occupy the junctional gap in the triads (1.4.3.2 B). T/SR associations are very extensive and occupied by evenly spaced rows of feet (1.4.3.2 C, D). In muscles from females the jSR cisternae are long, interposed with rare free SR segments and associated with uninterrupted double rows of feet (1.4.3.2 E, F). The feet are even spaced and in an orthogonal distribution (1.4.3.2 G). In Golgi "stained" tissue (see 5.2.1), $T$ tubules occupy the space between flat ribbon-like myofibrils and have occasional connections to the surface membrane (1.4.3.2 H), see 2.4.1 F. In muscles from females the wide jSR cisternae are very long (1.4.3.2 I), confirming thin sections views. In muscles from males the jSR cisternae are short and intercalated with frequent free T tubule segments (1.4.3.2 J). In thin sections this corresponds to short jSR segments associated with feet (1.4.3.2 K). This may be related to the function of $f \mathrm{~T}$ in allowing $\mathrm{T}$ tubule expansion in shortening (see 2.4.4). In deep etch images the extensive free SR membrane is occupied by a dense carpet of Ca pump proteins (1.4.3.2 L), see 2.3.3 A. Freeze-fracture exposes long stretches of cytoplasmic and luminal leaflets of T tubules (1.4.3.2 M, N). The cytoplasmic leaflet is occupied by DHPRs tetrads 
spaced at a distance double to the feet spacings (1.4.3.2 O, P), see 2.5.3.1 H. Franzini-Armstrong, 1972; FranziniArmstrong and Nunzi, 1983; Block et al., 1988; Appelt et al., 1991.

\subsubsection{Midshipman}

Midshipman fish belong to Porichthys (toadfishes). Muscles in their swimbladder have the property of maintaining a high frequency vibration for a prolonged period of time during the mating call. The muscle fibers have a well aligned cross striation (1.4.3.3 A) which is highly unusual because the $Z$ lines are extremely wide, while the $A$ bands have the standard structure (1.4.3.3 B, D). The SR is abundant and divided into segments by triads located at two sites within the A band (1.4.3.3 C). Muscles from actively "singing" specimens have frequent SR stack probably representing calcium entry units (1.4.3.3 E), see 2.10.2. Unpublished observations in collaboration with Simona Boncompagni and Matthew Kittelberger.

\subsubsection{Sand Dab}

The pacific sand dab (Citharichthys sordidus) is a bottom dwelling flat fish from the Monterey Ca. coast. Fin movements are controlled by muscle fibers composed of either three twitch types or two twitch and one slow tonic type. The fiber types are identified on the basis of T tubule structure and disposition in Golgi "stained" muscles, see 2.4.4. Fast twitch fibers have extensive junctional SR cisternae (1.4.3.4 A, B). Slow twitch fibers (1.4.3.4 C) have shorter junctional T and longer free T segments. Tonic fibers have a predominance of free T. Tubules and very short jT segments (1.4.3.4 D). Franzini-Armstrong et al., 1987.

\subsubsection{Glass Fish}

Glass fish are almost completely translucent (hence the name) due to the paucity of light scattering mitochondria. Sarcomeres in tail myotomes are highly ordered and at resting length they have almost complete thin/thick filaments overlap. Note moire' effects in a very thin section due to predominance of either thin or thick filaments in the section (1.4.3.5 A). In deep etch images of muscle in rigor cross bridges are visible (1.4.3.5 B) and various appearances are due to the position of the fracture plane relative to the filament arrays (1.4.3.5 C). Due to the vertebrate geometry (see 2.1.2.3) the disposition of rigor cross bridges is not as regular as in crayfish muscle. Actin target zones are variably decorated by from one to several cross bridges and the distance between target zones is variable (1.4.3.5 D, E).

The arrangement of SR and T tubules is highly stereotyped, with triads at the $Z$ lines and iSR segments in between (1.4.3.5 F). Varriano Marston et al., 1984.

\subsubsection{Sea Horse}

Fibers in the dorsal tail fin of "sea horses" have some of the standard features of fish muscle, but with an interesting variation. The myofibrils occupy only a part of the fiber, although the T tubules are connected to the surface (1.4.3.6 A, top image is from the work of EmilioVeratti, 1902), Within the myofibril region, triads and SR are essentially the same as in other fish muscles with triads at the $Z$ lines (1.4.3.6 B). Franzini-Armstrong, 2002.

\subsubsection{Goldfish}

Goldfish liver displays the same classical features as mammalian liver, but with some variations. As in zebrafish larvae (see 1.4.3.1.2 D) canaliculi are intra- rather then inter-cellular (1.4.3.7 A). Convoluted villi from the lining fibers dip into the same of Disse (1.4.3.7 B). Mitochondria-associated RER occupies a cell domain separated from that of SER and glycogen (1.4.3.7 C). Golgi system and secretary granules are well visible (1.4.3.7 D). 


\subsubsection{Reptiles}

\subsubsection{Turtle}

Turtles move ponderously but slowly. Strong muscles retract the legs under the carapace for protection. Interestingly, the free SR at A band level, mostly responsible for calcium uptake, is very scarce (1.4.4.1 A), but a double set of triads that are very short, but abundant, are located at the A-I junction with variable orientations (1.4.4.1 B,C). Overall there is an abundance of calsequestrin in jSR sacs. Other reptiles follow the same trend, in an exaggerated way (see 1.4.4.3 Nerodia and Boa snakes). Mitochondria are scarce. Page, 1968, Perni et al., 2016.

\subsubsection{Lizard}

Lizards move quite fast and at times they are very active. It is not surprising that the leg muscle fibers in a small lizard are well endowed with sarcotubular system elements. Triads are two/sarcomere and sometimes even doubled (1.4.4.2 A, B). The SR is abundant with frequent cisternae and often fenestrated, a configuration that combines optimal membrane surface and volume (1.4.4.2 A, B, C). Triads have extended rows of feet (1.4.4.2 D). Free SR is in multiple layers at the I-ZI level (1.4.4.2 E) and in single layer but quite abundant at the A band level (1.4.3.2 F). Lizard leg muscles were used in one of the early classic early experiments on e-c coupling by Andrew F. Huxley. Perni et al., 2016, Franzini-Armstrong, 2018.

\subsubsection{Snakes}

Some snakes swallow large prey whole, followed by a prolonged fasting and resting period with infrequent bursts of foraging activity. Nerodia (a northern water snake) and Boa constrictor follow this pattern (1.4.4.3 A, from Le Petit Prince by Antoine de Saint-Exupéry). The SR of Nerodia shows abundant fenestrated SR and an additional feature specific to these muscles: the extension of CASQ-containing SR along the free SR cisternae (1.4.4.3 B). The extensions occur both at the I band (1.4.4.3 C) and the A band level (1.4.4.3 D). In some fibers the triads are fragmented and connected to frequent CASQ filled free $S R$ sacs (1.4.4.3 E) a trend that is also seen in turtle muscles (see 1.4.3.1) as well as more strikingly in Boa muscles (see below). Longitudinal clusters of CASQ may be quite extensive and have been used to determine the 3D arrangement of CASQ polymers (see 2.5.2.3.1). The trend toward extension of calsequestrin in longitudinal SR is strong in Boa body muscle (1.4.4.3 F). Mitochondria-poor ("white" with CASQ extensions) and -rich (“red) are present (1.4.4.3 G, H). Close at al., 2014, Perni et al., 2016.

\subsubsection{Amphibia}

\subsubsection{Frog Twitch Fibers}

The majority of fibers in frog muscles are of the twitch type: they have action potentials, are innervated at a single motor end plate, and they respond to nerve stimulation with a twitch, a single contraction of fixed size. They have been extensively used for essential muscle physiology experimentation. They have a well defined cross striation (1.4.5.1 A) and well delineated myofibrils separated by SR, mitochondria, glycogen (1.4.5.1 B). The thin filament disposition is extremely regular near and at the $\mathrm{Z}$ lines $(1.4 .5 .1 \mathrm{C})$. The $\mathrm{M}$ line is well defined and the thin filaments are in the trigonal position in the overlap region of the $A$ band (1.4.5.1 D). Triads are located at the $Z$ lines (1.4.5.1 E) and they have parajunctionl feet, composed of RyR3 in addition to the standard double set of junctional feet (1.4.5.1 F).

The triads have two parallel rows of feet between $T$ and SR and indeed feet were first described in frog twitch fibers 
(1.4.5.1 G, H). Since the triads are at the $Z$ lines, the free $S R$ constitutes a single component covering the entire distance between triads (1.4.5.1 I). Golgi "staining"demonstrates that as in muscles for other sources, fibers belong to different categories: those rich in free SR (1.4.4.1 J), presumably fast twitch, and those scarce in SR (1.4.5.1 K), presumably slow twitch fibers.

See also 2.2.2 A, B; 2.1.3.1 C; 2.4.1 C; 2.5.1.1 A; 2.5.2.1 A; 2.5.2.3.1 B; 2.5.2.4 C; 2.5.2.3.1. E. Franzini-Armstrong 1970c, 1971; Franzini-Armstrong et al, 1973, 1975; Dulhunty, and Franzini-Armstrong, 1975, 1977.

\subsubsection{Frog Tonic Fibers}

Tonic fibers are used for prolonged holding of tension at little metabolic expense. They have no action potential, distributed neuromuscular junctions and the myofibrils are fused together (see 2.7.1). $T$ tubule network at the level of the $Z$ line form longitudinally oriented triads and dyads (1.4.5.2 A). The flat junctional face have limited arrays of orthogonally arranged feet (1.4.5.2 B, C). Freeze fracture images show extended SR membranes, mostly less abundant and organized than in twitch fibers (1.4.5.2 D, E). Plasmalemma at sites of PCs contains aggregates of DHPR tetrads associated with the junctional feet (1.4.5.2 F). Franzini-Armstrong, 1973b,1984.

\subsubsection{Birds}

Zebra finches are song birds. They have a fast beating heart and specially differentiated myocardium (see 1.5.2.2). Wing and leg muscles display minor variations on the basic structure of cross striated muscles. In the wing muscles a single or a doublet of triads with unusual longitudinal axis are located at the level of $Z$ the line (1.4.6 A, B). Small peripheral couplings are also present (1.4.6 C). Most mitochondria have an unusually large diameter (1.4.6 C), they are longitudinally oriented and they extend over many sarcomeres (1.4.6 B, D). Mitochondria disposition is random, but they are quite frequent (1.4.6 E). In leg muscles the triads have the traditional transverse orientation and position the edges of the $A$ band (1.4.6 F). As in the wing muscle the mitochondria are large, with no preferential location relative to the sarcomere bands and a close association with SR elements $(1.4 .6 \mathrm{G}, \mathrm{H})$ and overall they are less frequent than in the flight muscles (1.4.6 I, J). Leg muscle display elements of the calcium entry system or calcium entry units formed by stacked SR cisternae (1.4.6 K, see 2.10.2 A) see 2.10.2. See 2.11.3 for chick skeletal muscle. Franzini-Armstrong, et al., 1999; Perni et al., 201

\subsubsection{Mammals}

\subsubsection{Cat Extraocular Muscles}

Extraocular muscles control eye movements. They display the largest range of fiber type variations, from very fast twitch to slow tonic. In all organisms the fibers are endowed with a good complement of mitochondrial as befits their mode of constant operation. Mitochondria are frequent and often quite large (1.4.7.1 A-B, cat and frog). The size of myofibrils and thus the distribution of sarcotubular elements, an indication of contraction properties, are quite variable (1.4.7.1 C, cat). The overall structural range goes from fast fibers with multilayered SR and frequent triads (1.4.7.1 D, E, cat), to slow tonic fibers with poorly defined myofibrils at the A band level and limited SR (1.4.7.1 F, cat).

\subsubsection{Guinea Pig Leg Muscles}

Guinea pig leg muscles have an unusual feature: fibers of different types are almost entirely segregated into separate 
muscles, offering an advantage for the comparison of ultrastructure in Golgi "stained"fibers with the functional identification. Cross sections clearly illustrate the different contributions of junctional T tubule to the overall network in fast and slow twitch fibers (1.4.7.2 A-C), see also 1.4.7.2 D for a direct comparison at light and EM levels. Either T tubules or T plus SR are infiltrated by the Golgi silver-osmium precipitate allowing correlation between the two (1.4.7.2 E). The major difference in SR between fast and slow fibers is in the density of free SR membranes at the A band level (1.4.7.2 F top versus bottom). See 2.4.3 B, E for details of T tubules in longitudinal sections of guinea pig and relation to Vernier displacements of the cross striation. Ferguson and Franzini-Armstrong, 1988; Franzini-Armstrong et al., 1988a

\subsubsection{Mouse Fast Muscles}

EDL, FDB and Sternomastoid are "fast" mouse muscles because the majority of their fibers are of the fast twitch type, IIB/IXX. IIA and I type are also present, but in small numbers. Type IIX/IIB have none or scarce sub-plasmalemmal clusters of mitochondria. Intermyofibrillar mitochondria are located at the A-I junction in close rapport with the numerous triads (1.4.7.3 A and B). Type IIA fibers have frequent mitochondria at the Al junction but also numerous extensions along the $\mathrm{A}$ band (1.4.7.3 C). Type I, slow fibers are of smaller diameter and they have numerous mitochondria at all levels of the sarcomere as well as clusters at the edge of the fiber (1.4.7.3 D, E). The mitochondria at the A-I junction are closely apposed and linked to the triads (1.4.7.3 F-H). In the fast fibers the SR at the I-Z-I level is double layers (1.4.7.3 I, J) and highly convoluted (1.4.7.3 K), while the A band SR is in a single layer (1.4.7.3 L). See 2.7.2. Boncompagni et al., 2010.

\subsubsection{Mouse Slow Muscles}

The soleus is a postural muscle requiring fatigue resistance. In the mouse, soles has approximately $50 \%$ type I slow twitch fibers and 50\% type IIA fast twitch oxidative, both highly endowed with mitochondria. Type I fibers are small in diameter and in addition to the set of transversely oriented mitochondria associated with triads at the A-I band junction they have frequent large longitudinal extensions (1.4.7.4 A and B) and clusters of mitochondria are at the fibers' edges (1.4.7.4 C). Triads are less abundant than in the fast twitch fibers (1.4.7.4 D). Type IIA fibers have frequent triads at the A-J junction and associated mitochondria in the I band regions (1.4.7.4 E, F). Longitudinal mitochondria extensions and peripheral clusters are less frequent than in the I fibers. Triads have more extended jSR cisternae than in type I (1.4.7.4 G). (Compare also with type IIA fibers in the EDL 1.4.7.3 C). Boncompagni et al., 2010.

\subsubsection{Rat Sternomastoid}

Muscle fibers in rat sternomastoid muscle can be classified into three categories on the basis of the fiber size and the content of mitochondria and lipids. Fast twitch, fatigable, glycolytic fibers are the largest, they have the smallest content of mitochondria, limited to the I band region, and are associated with few capillaries (1.4.7.5 A). The muscle area is pale in their proximity, leading to the appellation of "white". Fast witch, oxidative-glycolytic fibers, with intermediate resistance to fatigue have intermediate size, mitochondria distributed at both I and A bands, few peripheral mitochondria and some associated capillaries. They are also named "pink' or 'intermediate' (1.4.7.5 , B, C). Slow twitch, fatigue resistant, oxidative fibers are of smallest size, they are highly endowed with mitochondria, including clusters at the fiber's edge, they have numerous lipid droplets and they are associated with a large number of capillaries. They are located in the red regions of the muscle (1.4.7.5 D,E). In all fibers mitochondria at the I band level are elongated and form a continuous network (1.4.7.5 F). See 1.4.7.3 and 1.4.7.4 folders for similar images in mouse muscle. Sally Johnson, unpublished. 


\subsubsection{Vertebrate Cardiac Muscles}

\subsubsection{Purkinje Bundle}

Cardiac Purkinje cells are components of the specialized conductive system fibers that carry a signal from the base to the tip of the ventricle. They are segregated from the working myocardium by an endothelium and connective tissue shield (1.4.8.1 A, rabbit). Longitudinal continuity is through intercalated discs similar to those in the working myocardium (1.4.8.1 $\mathrm{B}$, rabbit), but more frequently involve a complex association with desmosomes and the intermediate filament network (1.4.8.1 C, rabbit), Common features are the paucity of myofibrils; the glycogen filled cytoplasm and extensive lateral connections by gap junctions and occasional desmosomes (1.4.8.1 D, E, cat). The intercalated disk involves extensive interdigitation between the connecting cells (1.4.8.1 F, cat, see also 1.4.8.1 C, rabbit). Di Maio et sl., 2007.

\subsubsection{Bird heart}

\subsection{Chick Corbular jSR}

As in other birds (see 1.4.8.2.2) myocardial cells from chicken to not have traverse tubules. In the embryonal stages, before hatching, they have numerous PCs (1.4.8.2.1 A ) and in the post hatching and adult myocytes they have aggregates of ryanodine receptors organized into small clusters constituting specialized SR domains called corbular SR ,that function as calcium release sites. In cross and longitudinal sections corbular SR domains as small dark sites (1.4.8.2.1 B, C) distributed as a collar around the myofibrils at the level of $Z$ lines (1.4.8.2.1 D-F). In longitudinal sections continuity of the SR network and its specialized corbular domains are more obvious (1.4.8.2.1 G). DHPRs are clustered at sites of PCs (1.4.8.2.1 H) but are NOT organized in tetrads. See 2.5.3.2. Sun et al., 1995; Perni et al., 2012.

\subsection{Finch PCs and EJSR}

The myocytes of fast beating avian hearts (finch and hummingbird) are primary examples of myocardial cells that lack $T$ tubules, but can activate fast calcium release. As first suggested by Joachim R. Sommer and colleagues in 1994, the mechanism involves and initial voltage dependent activation at PCs through the interaction of CV1.2 with RyR2 and subsequent spread by CACR through very extensive RyR2 bearing elements called EJSR. Despite the small diameter of the finch myocytes (1 4.8.2.2.A) the cells are endowed with a high content of PCs and extensive EJSR elements at the Z line level (1 4.8.2.2 B, C). The end to end distance between contiguous EJSR elements is very narrow, an indication that $\mathrm{CICR}$ is a vey likely transmission mechanism (14.8.2.2 D). Although less extensive, the corbular SR of chick myocardium (see 1.4.8. 2.1) is functionally equivalent to EJSR.

The position of PCs on the plasmalemma is detected by freeze-feature images in which the location of underlying $Z$ lines is very clear (1.4.8.2.2 E). Each PC location is marked by a cluster of DHPR particles (1.4.8.2.2 F). The particles are clearly grouped within a limited area, but they are NOT arranged into tetrads (1.4.8.2.2 G).

As in other hearts, innervation is by non myelinated nerves that release they secretory vesicles content affecting many myocytes, without differentiation of a synaptic structure (1.4.8.2.2 H). Franzini-Armstrong et al., 1999; Perni et al., 2012.

\subsubsection{Frog Heart}

Frog heart has 3 chambers: two atria and one ventricle. Interestingly, the atria have a more robust e-c coupling apparatus then the single ventricle and the latter relies heavily on calcium entry from the extracellular space. Atrial PCs are relatively frequent (1.4.8.3 A and B) and have well defined RyR2 foci (1.4.8.3 C, D). DHPRs in the plasmalemma are clustered in small groups corresponding to $P C$ sites (1.4.8.3 E-G). PCs are present $n$ the ventricle, but less frequent than in the atria 
(1.4.8.3 H-J). Clusters of DHPRs are small and infrequent (1483 K, L). Tijskens et al., 2003a.

\subsubsection{Lizard Heart}

Lizard is a reptile with very small myocardial cells devoid of T tubules and corbular SR, but rich in peripheral couplings (1.4.8.4 A, B) associated with DHPR clusters (1.4.8.4 C, D). Intercalated disks are of the standard stepwise fashion (1.4.8.4 E, H). Perni et al., 2012.

\subsubsection{Rat Heart}

Cardiac myocytes in rat have a regular alignment of mitochondria in single or multiple longitudinal columns between the myofibril. Most mitochondria have the length of one sarcomere, and are interrupted by $T$ tubules and dyads at every $Z$ line (1.4.8.5 A-C). Longer or shorter mitochondria are less frequent (1.4.8.5 D). Large aggregates of mitochondria are frequent at the cell edges (1.4.8.5 E). The SR is less abundant than in mouse cardiac (1.4.8.5 F). Due to their positioning between the myofibrils mitochondria and dyads come to close contacts at the $Z$ line $(1.4 .8 .5 \mathrm{G})$ favoring an indirect transport of calcium from the SR to mitochondria at every heart beat. Since many dyads are formed along the T tubule, multiple dyad/mitochondrion contacts may occur $(1.4 .8 .5 \mathrm{H})$. An unusual feature of mitochondria was firsts discovered in rat cardiac myocytes and subsequently confirmed in other mammalian heart (see 2.8.5 and 1.4.8.6). This is the existence of thin extension of the mitochondria to form tunnels that provide communication between the organelles at some distance (1.4.8.5 I, J). During differentiation T tubules, marked by ferritin, have an irregular disposition and wide lumen (1.4.8.5 K). All images are from ventricular cells. Ramesh et al., 1998; Sharma et al., 2000; Huang et al., 2013.

\subsubsection{Mouse Heart}

Two features differentiate mouse from rat (see 1.4.8.5) cardiac muscles. One is the relative abundance of free SR and the other is the enhanced motility of mitochondria. Extensive lacy networks of free SR are visible in most thin sections of mouse myocytes (1.4.8.6 A-D, see also1.4.8.6 E)). Differently from skeletal muscle the SR is continuous longitudinally, since dyadic junctions with the T tubules do not interrupt the free SR (1.4.8.6 E, compare with 1.4.8.6 B, D). T tubules run transversely (1.4.8.6 F), but the free SR bypasses them. Mitochondria extensions (nanotunnels) are quite frequent (1.4.8.6 G) and become more frequent and extensive under experimental conditions inducing calcium imbalance (1.4.8.6 H, I). Intercalated discs are sites of active interdigitation between participating myocytes, They are most obvious in transverse sections (1.4.8.6 J). Lavorato et al., 2017.

\subsubsection{Rabbit and Dog Heart}

T tubules have a large diameter (1.4.8.7 A,B rabbit; C,D dog). Dyads are extensive and located in proximity of $\mathrm{T}$ tubules (1.4.8.7 E,F rabbit; G dog). Free $\mathrm{SR}$ is of limited extent (1.4.8.7 H rabbit) and hard to detect (1.4.8.7 I, dog). Peripheral couplings are present (1.4.8.7 J dog). The intercalated disc has the usual step-wise configuration (1.4.8.7 $\mathrm{K}$ rabbit), but the steps are narrow in dog (1.4.8.7 L). See examples in smaller animals (see 1.4.8.5 and 1.4.8.6). Sleeper et al., 2002; Lavorato et al., 2020.

\subsubsection{Rat Cerebellum}

Purkinje cells in the cerebellum have an unusual arrangement of IP3Rs. The channels are inserted at high density and in semicrystalline arrays into the membranes of ER stacks constituted of multiple lamellae (1.4.9 A and B), see 2.9.3. Most of the stacks are in close proximity to mitochondria and apparently linked to them (1.4.9 C-E). Even tough the proximal stack 
membrane is very close to the mitochondria surface, IP3Rs are clearly not associated with the outer mitochondria membrane, because they are located on the side of the stack lamella that faces away from the mitochondrion. In collaboration with Venkat Ramesh lyer

\subsubsection{Smooth Muscle}

E-C coupling machinery is present in the phasic smooth muscle cells of the guinea pig urinary bladder, although they are far less extensive than in skeletal muscle. The cells are small, and filled by the contractile apparatus except for a central core (1.4.10 A). Small non myelinated nerves are distributed between the cells, but there are no specific neuromuscular junctions $(1.4 .10 \mathrm{~B})$. T tubules are absent The surface membrane is occupied by dense patches of adhering junctions, connected to thin filaments, interspersed with numerous caveolae and rare, small peripheral couplings containing few RyRs (1.4.10 C, D). Caveolae are located in longitudinal strips alternated with smooth membranes where the adhering junctions are located (1.4.10 E). Very small groups of prominent particles identifiable as DHPRs are located within the caveolar domains (1.4.10 F, G). The particle clusters are small and the particles are NOT arranged into tetrads $(1.4 .10 \mathrm{H})$. Correlated positioning of RyRs and DHPRs, as well as calsequestrin, is confirmed by immunolabeling (1.4.10 I). Moore et al., 2004.

\subsubsection{In vitro}

$\mathrm{BC} 3 \mathrm{H} 1$ is a permanent cell line derived originally from a mouse brain tumor but later found to express skeletal muscle isoforms of $\mathrm{CRU}$ components. Upon differentiation the cells poorly develop structural skeletal muscle components (myofibrils and T tubules), but CRU proteins are efficiently expressed and assembled into peripheral couplings (PCs) with associated RyRs (1.4.11 A) and extensive plaques of DHPRs in the plasmalemma (1.4.11 B, C). Association of DHPRs plaques with RyRs in PCs is demonstrated by the extremely accurate grouping of DHPR particles into tetrads within the plaques (1.4.11 D, E). Dots placed in the center of tetrads graphically highlight tetrad arrays and demonstrate that small adjacent clusters have different orientations and thus are assembled independently (1.4.11 F). Protasi et al., 1997; Holtzer et al., 1997.

\subsection{Differentiation}

\subsubsection{T tubules}

T tubules of differentiating skeletal muscle are traced either by labeling the membrane with fluorescent compounds (1.5.1 A-C, see 5.2.3) or by tracing $T$ lumen by the $K$ ferrocyanide technique (1.5.1 D-F, see. 5.1.3). At early stages of development the first T tubule imaginations appear at the fiber surface and penetrate inward (1.5.1 A). Frequency of tubules increases with developmental age, but in mouse the predominant orientation is still longitudinal for several post natal days $(1.5 .1 \mathrm{~B})$. The $\mathrm{SR}$ is also introduced at this stage, with the tendency with the formation of CRUs arranged in a transverse structural orientation (1.5.1 C, see also 1.5.2). In the postnatal differentiation new $T$ tubules are formed by caveolar invaginations (1.5.1 D, E) and for as period of time (up to a month in mouse muscle), the new $T$ tubules have a mixture of longitudinal and transverse orientations. Franzini-Armstrong, 1991; Flucher et al., 1993; Takekura et al., 1993. 


\subsubsection{Triad Positioning}

Specific location of CRUs relative to the sarcomere bands precedes the transverse orientation of $T$ tubules networks in developing mouse muscle. At E15 T tubule have not yet developed (see 1.5.2 A) and CRUs are peripheral couplings (1.5.2 A), At E 16 when T tubules are just beginning and are totally longitudinal (see 1.5.1 A ) RyR hot spots indicating positioning of newly formed dyads/triads occupy double transverse bands (1.5.2 B). At E 17, when the T tubules are still mostly longitudinal, the transverse double bands of triads are very clear (1.5.2 C). The trends continues at E18 and D1, while T tubules gradually acquire transverse bands (1.5.2 E, F). 1.5.2 G summarizes then stages in triad formation. Takekura et al., 1994a, 2001.

\subsubsection{Triad Maturation}

Formation of triads and accrual of the two major elements in e-c coupling (RyR and DHPR) are independent of the presence of the other component (1.5.3 A). A scheme supported by a variety of observations in WT and null mutants suggests that the formation of skeletal muscle CRUs is initiated by the docking of RyR to sarcolemma/T tubules, followed by acquisition of CASQ and its link to triadin/junctin. This establishes an appropriate environment for trapping RyR and DHPR. Finally, the latter two components link to each other in a stereospecific manner (1.5.3 B). The initial associations between SR and T tubules to form triads involves primitive longitudinally oriented T tubules (see 1.5.1 and 1.5.2) so the axis of young triads are predominantly longitudinal. With time the triads gradually rotate until they achieve the final transverse orientation (1.5.3 C). Takekura et al, $1995 \mathrm{a}$ and b; Protasi et al., 1996, 1997, 1998a; Flucher and Franzini-Armstrong, 1996; Felder et al., 2002; Franzini-Armstrong, 2004a; DiBiase and Franzini-Armstrong, 2005.

\subsubsection{Myotubes and Myofibrils}

Development of skeletal muscle fibers occurs in two (or three for larger animals) successive waves of myoblast proliferation and the differentiation of their progeny into myotubes and eventually myofibers. In the first wave a set of homogeneous primary myotubes is formed (1.5.4 A). The myotubes are in close proximity to each other and connected by gap junctions. A successive sets of myotubes, the secondary myotubes originate from a second set of proliferating myobasts. At this stage the primary motives move apart and allow clusters of secondary motives to adhere closely to their surface (1.5.4 B). Primary myotubes communicate with each other and with the secondary myotubes via gap junctions (1.5.4 C), but fully differentiated myofibers are electrically isolated from each other. The primary and secondary positioning is highly significant because it determines the final fates of the fibers involved in terms of functional types.

Myofibril assembly occurs quite rapidly a soon as myobrillogenesis is initiated. It involves several gradual stages of increased ordered in the arrangement of myofilaments and in the structure of the $Z$ line (1.5.4 D). Wiping et al., 1992; Holtzer et al., 1997.

\section{Part 2 Structure-Function}

\subsection{The Contractile Machinery}

\subsubsection{Myosins}

Myosins comprise a large family of proteins that translate along actin filaments by motor domains with the common characteristics of ATPase function and affinity for actin. Lever arms, one for each head, allow the movement of the 
myosin motor along actin filaments.

Myosin II, the component of muscle sarcomeres, is a dimeric, double headed molecule with fairly short lever arms and a very long tail that forms a coiled coli helix and polymerizes to constitute the core of muscle's thick filaments. It was the first myosin to be identified (2.1.1.A.lower portion).

Myo iPi (2.1.1 B) is a yeast (Saccharomyces cerevisiae) myosin II with a long kinked tail. It is involved in cleavage furrow of dividing cells.

Myosin V (2.1.1 C,D,E) is responsible for actin-based intracellular transport and positioning of certain membrane bound vesicles, endoplasmic reticulum and mRNA-containing particles. It has a cargo binding tail and long lever arms that makes it ideal for study of myosin processivity.

Myosin VI $(2.1 .1 \mathrm{~F})$ is a reverse-direction actin-based motor that is prevented from dimerizing unless forced. Full-length myosin VI is capable of forming a stable, processive dimer when monomers are clustered, moving distances up to 1-2 $\mu \mathrm{m}$ in $~ 30 \mathrm{~nm}$ (center of mass), taking surprisingly large $(30-36 \mathrm{~nm})$ when dimerized, hand-over-hand steps.

Myosin I (2.1.1.G), with a single head and short lever arm includes a variety of molecules involved in dynamics of cell organelles and membranes.

Park et al., 2006; Syed et al.,2006; Mukherjea, et al., 2009; Fang et al., 2010; Park et al., 2006;McIntosh et al., 2026.

\subsubsection{Filaments and Cross Bridges}

\subsubsection{Myosin Filaments}

Thin (actin) and thick (myosin) filaments are isolated by gentle homogenization of muscle bundles in the presence of ATP and low calcium to allow relaxation of cross bride attachments. Both filaments are part of the crude homogenate fraction (2.1.2.1 A). All thick filaments have a helical arrangement of myosin heads on their surface, but a with quite variable parameters due to the number of molecules and their positioning. The superficial arrangement of cross bridges is most easily disarrayed in filaments from skeletal muscle, so arthropod filaments have been studied in most detail (2.1.2.1 B, C). The center of the filaments, where myosin molecule abut tail to tail is smooth, no cross bridges protrude from the surface (2.1.2.1 B). On the two sides are myosin head disposed in helical arrangement with a reversed polarity at the center of the sarcomere. Polarity reversal is essential to sarcomere shortening. The polarity of thin filaments, which is revealed by their interaction with myosin cross bridges reverses at the $Z$ lines and in the center of the sarcomere.

\subsubsection{Actin Filaments}

Actin filaments are long polymers of $\mathrm{G}$ actin monomers arranged in a helix with an axial translation of $27.5 \mathrm{~nm}$ (half of an actin monomer). Two strands twist around each other to form a helix with repeat of 350-380 nm. The in vivo length of actin filaments is determined by the sarcomeric structure. It varies between 1-2 $\mu \mathrm{m}$ in vertebrate sarcomeres, and up to $100 \mu \mathrm{m}$ or more in muscles of invertebrates. Filaments can be quite long when polymerized in vitro in the absence of capping proteins.

Isolated actin filaments ( $50 \mathrm{~nm}$ in diameter) are well visible in negatively stained images (2.1.2.2 A) and the repeat of the long helix is detected at higher magnification (2.1.2.2 B).

In the absence ATP the muscle is in a "rigor" condition in which all myosin cross bridges are attached to actin filaments in a "strong binding", durable configuration (see 2.1.2.3 A-J). When myofibrils from crayfish muscle in rigor are exposed to hyperosmotic solutions (e.g., $0.6 \mathrm{M} \mathrm{KCl}$ ) the backbones of myosin filaments dissociate and the thin filaments are also 
detached from the $Z$ lines, while the cross bridges remain in the original rigor configuration. The resulting "natively decorated" thin filaments offer a good deal of information on actin-myosin interaction. Periodic cross bridges forming oriented arrowheads due to the angle of cross bridge attachment, decorate the actin filaments except for the segments originally located in the bridge free region of the A band and in the I band. Occasionally a small group of filaments maintains its association with a remaining section of $Z$ line, showing that all arrowheads point away from the $Z$ line. (2.1.2.2 C). The cross bridges are attached at intervals that equal the period of the actin filament helix, thus marking the position of the actin target zones. Each target zone receives either one or two cross bridges depending of the position of the cross bridges arising from the thick filament surface (2.1.2.2 D and E). Negatively stained images show more detail than the shadowed ones (2.1.2.2 E), including the fact that the two heads of s single myosin molecule can be resolved (2.1.2.2 F). See 2.1.2.3 I, J. Varriano-Marston et al., 1984; Northrop et al., 1986; Bard et al., 1987; Loesser and FranziniArmstrong,, 1990; Bard and Franzini-Armstrong, 1991.

\subsubsection{Rigor Cross Bridges}

In the absence of ATP muscle is in the stable rigor configuration in which all thick filaments cross bridges that are in the appropriate attachment to target zones in the immediately adjacent actin filaments. The resulting patterns have been used by Michael C Reedy to define the rules of actomyosin interactions within the constrains of sarcomeres. Given the cross bridge freedom to move axially and radially from their site of origin on the surface of the thick filament, all rigor cross bridges find attachment sites at actin target zones. So while relaxed muscle is dominated by the periodic disposition of cross bridges on the myosin helices (see 2.1.2.1 B) rigor muscle is dominated by the periodicity of helix parameters in actin filaments.

Visibility of cross bridges in thin sections depends on section thickness and orientation of the section plane relative to the filaments arrays. In a fairly thin sections (2.1.2.3 A) areas illustrating alternate thin and thick filaments alternate with others that show only thin filaments. Some overlap of filaments is present. A VERY THIN section shows two thin filaments separating thick filament profiles and a clear view of the cross bridges (2.1.2.3 B). Due to the relative position of thin and thick filaments (see $2153 \mathrm{~B}$ ) and to matching helix parameters of thin and this filaments, cross bridge distribution his considerably more regular in arthropod (2.1.2.3 C, D) than in vertebrate muscles. Holding the images at eye level and glancing across the sarcomere clearly reveals the dominating actin-based periodicity.

Shadowed images of cryofractured deep etched sarcomeres are equivalent to very thin section images, showing individual cross bridges. In skeletal muscle (from glass fish) actin target zones are decorated by a variable number of attached cross bridges and the two sides of the same filament do not match (2.1.2.3 E-G). Periodicity of the actin target zones is obvious wherever the fracture plane coincides with thin filaments $(2.1 .2 .3 \mathrm{H})$.

In crayfish, an arthropod, periodicity of the actin target zones is emphasized by the fairly even distribution. of decorating cross bridges and the symmetry of the two sides of the thin filaments (2.1.2.3 I, J ). This is a direct result the dimeric position to thin filaments. See also "natively decorated"filaments isolated from rigor muscles (2.1.2.2 C). Muscles from arthropods have an additional advantage advantage: the matching of actin and myosin helical periodicites at every third turn. As a results, the rigor cross bridge disposition is semicrystalline. In skeletal muscles the helix parameters do not match and the actin filaments must interact with three myosin filaments. As a result the rigor pattern is apparently disordered, offering a higher probability of interaction when filaments slide past each other in a contraction. 
In muscles from arthropods (Drosophila 2.1.2.3 C, Lethocerus 2.1.2.3D) very thin sections illustrate alternate rows of thin and thin filament in some areas and a layer of thin filaments only in others. Varriano-Marston et al., 1984; Bard et al., 1987.

\subsubsection{Active Cross Bridges}

The movements of cross bridges are not coordinated during active contraction, Thus averaging techniques (such as X-ray diffraction ) have failed to identify conformational changes of individual cross bridges during during the active cycle. The currently accepted model of contraction, suggested by the atomic structures of myosin subfragment 1 (the myosin "head", Rayment et al., 1993) and its association with of the polymerized actin filament (Holmes et al., 1990) is that the motor domain maintains a single orientation relative to the actin filaments, while the light chain domains constituting the lever arms produce the working stroke.

Rapid freezing techniques allow the capture of actively moving cross bridges at a moment in time. Keiko Hirose working in Yale .E.Goldman laboratory, obtained images during the early steps in force generation when rigor fibers were activated by the rapid release of ATP captured structural changes in the cross bridge that precede and accompany generation of force (2.1.2.4 A and B). Thomas Lenart, in the same laboratory revealed the rapid overall changes in configuration (2.1.2.4 C) and movement of mass toward thin filaments in the activation of relaxed fibers by release of calcium from a caged compound.

More striking were the results obtained taking advantage of the semi crystalline order of cross bridges of Lethocerus indirect flight muscle (2.1.2.4 D) and the ability of the muscle to sustain an isometric contraction at high calcium concentration (2.1.2.4.E). revealed the rapid overall changes in configuration (2.1.2.4 $\mathrm{C}$ ) and movement of mass toward thin filaments.

Using EM tomographic analysis Taylor et al., visualized the first time the structural conformations of cross bridges during the contraction cycle (2.1.2.4.F). The analysis demonstrates that a minor change at the actin binding site and a swing of the lever arm occur during cross bridge action. This has been later extended by a series of publications from the TaylorReedy collaborations. Hirose et al., 1993, 1994; Lenart et al., 1996; Tregear et al., 2004; Wu et al., 2010, 2012.

\subsubsection{Skeletal Muscle Sarcomeres}

\subsubsection{Sarcomere Bands}

The sarcomere is the unitary structural component of the myofibrils. By convention it is defined as the distance between successive $Z$ lines. Structural details of sarcomeres depend on the disposition of thin and thick filaments and their anchoring and cross linking components (2.1.3.1A). The I bands, on either side of the $Z$ line, are constituted of thin (actin) filaments. The central band of the sarcomere, the A band, is constituted of aligned thick (myosin) filaments. The thin filament interdigitate with the thick filaments in the overlap region of the $\mathrm{A}$ band. The $\mathrm{H}$ zone is the central $\mathrm{A}$ band region not reached by thin filaments. The length of the $\mathrm{A}$ band is constant, but I band, overlap region and $\mathrm{H}$ zone depend on the sarcomere length, which varies with contraction and/or stretch. The narrow band with lower density bisecting the $A$ band is due to the alignment of the central bridge free region of the thick filaments (see $2121 \mathrm{~A}$ ). The bridge-free region is in turn bisected by a dense $\mathrm{M}$ line (missing in some muscle fibers (see 2.2.4). The thick filament of vertebrate muscles have a constant length and are held in aligned position by the $\mathrm{M}$ line, so that the edges of the $\mathrm{A}$ band are very sharp (2.1.3.4 B). The alignment of the last set of cross bridges in sarcomeres are best preserved by rapid freezing (2.1.3.4 $\mathrm{C})$. The edges of 
the $\mathrm{H}$ zone are sharply delimited indicating the equal lengths of most thin filaments, although a few are broken to shorter lengths (not shown). Cross bridge positioning and actin- and myosin- associated proteins are responsible for fine periodicities across $\mathrm{A}$ and I bands that are visible by holding the image horizontal and glancing across the sarcomere. Consult Myology for further details.

Association of cytoplasmic proteins with the thin filaments defines the position of two less well defined cross lines within the I band, the $\mathrm{N}$ lines (2.1.3.1 D). Ferritin molecules infiltrated in a skinned fiber adhere to the $\mathrm{N}$ lines (2.1.3.1 E). FranziniArmstrong,, 1970.

\subsubsection{Thin Filament Lengths}

All skeletal muscles. regardless of their origin have the same length of thick filaments $(1.6 \mu \mathrm{m})$ and width of the central bridge free region. However, width of $Z$ lines (see 2.2) and length of thin filaments and are variable. The length of thin filaments is dictated by the excursion of sarcomere lengths expected in the in vivo performance of the muscles. The white tail muscles of the glass fish bend very little in the swimming motion so sarcomere lengths vary by little and the length of thin filaments is quite similar to the length of half $A$ band. At resting length, the I band length is very short (2.1.3.2 A). In another small fish the resting length of the I band it only slightly longer (2.1.3.2 B) and extrapolated resting I band length in frog and rabbit indicate clearly longer thin filaments (2.1.3.2 C and D). A double set to triads in each sarcomere (at the A-I junction) is present in fibers with longer thin filament lengths, as first noted by Sally Page. Using the length of the A band as reference, the lengths of thin filament can be estimated to vary between 1.5 and 2.2 $\mu \mathrm{m}$ (2.1.3.2 E). These data are approximate, since the measurements were taken from electron micrographs where it is known that the tissue is affected by varying degrees of shrinkages during embedding procedures. Lenart et al., 1996

\subsubsection{Band patterns/Sarcomere Lengths}

Sarcomere length changes either as a result of passive stretch and shortening in relaxed muscle, or due of active shortening in contracting muscle. The structural changes with sarcomere length follow the sliding filament model in which thin and thick filaments slide past each other without changes in their length. Width of overlap zone, $\mathrm{H}$ band and I band are variable, but width of $A$ band, bridge free band and $M$ lines are constant (2.1.3.3 $A$ and $B$ human, $C$ frog). The resting sarcomere length is at maximum overlap between the thin filaments and the cross bridge regions, but the overlap excludes the bridge free regions (2.1.3.3 D frog; E, fish; F drosophila; I crab). Shorter sarcomere length, requiring an active contraction, allow thin filaments from the two halves of the sarcomere to interdigitate with each other (double overlap), so that the ratio of thin to thick filaments is doubled $(21.3 .3 \mathrm{G}, \mathrm{H}$, frog). With further shortening the edges of the $\mathrm{A}$ band abut against the $Z$ line (2.1.3.3 J rat). Experimentally induced shortenings below that length usually result in damage and cannot be reversed (2.1.3.3 K, L). Franzini-Armstrong, 1970a.

\subsubsection{Section Thickness Effects}

Thin and thick filaments are closely packed and arranged in a semicrystalline pattern. Their visibility in longitudinal sections varies greatly from one area to the other, depending on the orientation of the section plane and on the thickness of the section, as shown in 2.1.3.4 A (frog). A very thin section with the orientation indicated as "1" in figure 2.1.3.4 A and in almost perfect longitudinal orientation shows two sets of thin filaments alternated with the thick ones (2.1.3.4 B glass fish). A different orientation, as "2" and a less precise alignment results in the image of figure 2.1.3.4 C, where the pattern varies in different areas. Most commonly used sections are thicker ("3" and "4" in 2.1.3.4 A) and less precisely aligned 
longitudinally. In these the thick filament profiles are superimposition of several filaments, so their apparent longitudinal continuity is false. (2.1.3.4 D-F frog). Fine cross periodicities due to structural details of thin and thick filaments are better visible in the ticker sections (2.1.3.4 E, frog). Varriano-Marston et al., 1984.

\subsubsection{Thin/Thick Filaments}

The disposition of filaments depends by their position in the sarcomere. Two segments of skeletal muscle sarcomeres have the most ordered arrangements. One segment is the A band, dominated by the hexagonal disposition of thick filaments (2.1.3.5 A) that is in turn stabilized by M line cross links (2.1.3.5 B, C), see 2.2.4 B, D. Within the A band's overlap zone the precise location of thin filaments in the trigonal position (at the center thick filaments triangles) determines the double hexagonal thin/thick filaments array specific of skeletal muscle (2.1.3.5 D) with a overall ratio of 2:1 thin/thick filaments. A second ordered segment is at the $Z$ lines, where alpha actinin cross-links hold the thin filaments in an orthogonal array (2.1.3.5 E). The orthogonal disposition is imposed on thin filaments within a segment delimited by $Z$ line on one side and $\mathrm{N} 1$ line on the other (see 2.1.3.1 D). Within the rest of the I band the thin filaments are somewhat disordered as they shift from orthogonal to hexagonal disposition.

In greatly shortened sarcomeres the center of the A band has an increased density due to the double overlap of thin filaments from the two side's of the sarcomere (2.1.3.5 F) and the thin/thick filaments ratio is up to $4: 1(2.1 .3 .5 \mathrm{G}, \mathrm{H})$. All images in this section are from frog muscle, with the exception of 2.1.3.5.H from a finch. See 2.1.5.3 for comparison with thin filament disposition in arthropods. Franzini-Armstrong, 1973; Varriano-Marston, 1987.

\subsubsection{Myofibrils Cross Striation}

\subsubsection{Myofibrils}

The actomyosin systems of muscle fibers are arranged in myofibrils, longitudinal columns that run along the length of the fiber (2.1.4.1 A) and are separated from each other by thin layers of sarcoplasm carrying sarcoplasmic reticulum, $T$ tubules, mitochondria etc. (2.1.4.1 B, C). The functional effect of the myofibrillar organization is to allow close proximity between the actomyosin system and the sarcotubular system that delivers calcium for activation of contraction and sequesters calcium for relaxation. To this effect the size and shape of myofibrils are modulated in accordance with the fibers' requirement. Twitch and tonic fibers of vertebrates have small and large myofibrils respectively (see 2.7.1). Very fast fibers have narrow ribbon-like myofibrils, so that diffusion of calcium from the SR to the actin filaments occurs over short distance. This is the case of the fast muscle in the toadfish swimbladder. (2.1.4.1 D) and of the dragonfly flight muscle whose isolated myofibrils are flat ribbons (2.1.4.1.E). Fast indirect flight muscle of some insects are called "fibrillar" because they have small diameter round myofibrils $(2.1 .4 .1 \mathrm{~F})$. The myofibrils on the very fast muscles of a small copepod seem to be an exception because they are quite large (2.1.4.1 G). However in this case SR and T tubule elements penetrate into the heart of myofibrils thus establishing the essential proximity to acto-myosins, see 1.3.2.1 A Franzini-Armstrong, 1972; Appelt et al., 1991; Takekura and Franzini-Armstrong, 2002; Glaser et al., 2018.

\subsubsection{Cross striation, Vernier}

The structure of skeletal, vertebrate cardiac and body muscles of invertebrates is dominated by periodicity of structural elements at right angle to the fiber long axis, the cross striation. The cross striation is generated by the combination of two factors. One factor is the basic feature of the myofibril (2.1.4.2 A, fish) in which A band, $Z$ lines and other features are cross 
oriented. The second feature is the alignment across the fiber of the myofibrils' striation (2.1.4.2 B, frog) and of the accompanying CRU locations. In skeletal muscle the cross alignment is perfect over large areas of the fiber, but occasionally a fault line appears at the border between myofibrils in the form of a vernier displacement, This is due to the fact that over a short distance the myofibrils on one side contain one more sarcomere than those on the other side. The length of vernier misplacements range from 2 to 10-20 sarcomeres. The vernier fault is visible in phase contrast image of mouse fibers (2.1.4.2 C), in mouse fibers immune labeled for triad positions (2.1.4.2 D), and in electron micrographs (2.1.4.2 E, mouse soleus). Vernier sites are subject to stress, resulting in $Z$ line streaming in aged and pathological muscle fibers (see 4.1.2). Golgi "staining"reveals loss of transverse positioning of SR and T tubules at Vernier sites (2.1.4.2 F). See 4.1.2. Franzini-Armstrong, 2002; Boncompagni et al., 2010.

\subsubsection{Arthropod Muscles, Myofibrils and Sarcomeres}

\subsubsection{Sarcomere Bands}

Body muscles of arthropods have well defined sarcomeres (2.1.5.1 A) with a basic pattern of overlapping actin and myosin filaments similar to skeletal muscle. However, although the bridge-free regions of thick filaments are mostly well aligned in the center of the sarcomere, the $\mathrm{M}$ band is missing (2.1.5.1 C) and the edges of $\mathrm{H}$ zone and particularly of $\mathrm{A}$ band are not sharp (2.1.5.1 B), indicating variability on the filament lengths. Additionally $Z$ line structure differs from that of vertebrates. One major difference from vertebrates is a relatively large overall variability of A band length even within sarcomeres of adjacent myofibrils (2.1.5.1 D, E, F). The length of the thin filaments is coordinated with that of thick filaments; the result is a fairly large variation is resting sarcomere lengths between fibers with different functional characteristics and some disorder of the cross striation (2.1.5.1 F). Insertion of new sarcomeres in the middle of the existing ones and their growth to match the fully developed length is responsible for obvious variations in filament lengths even within the two sides of the same sarcomere.

Rate of shortening in crustacean muscles is not based on fundamental properties of the myosin II ATPase, but it depends instead on filament and sarcomere lengths. The muscles of a small copepod are all fast, but resting sarcomere lengths and thus rate of shortening vary by a factor of two (2.1.5.1 G). Franzini-Armstrong, 1970a; Franzini-Armstrong et al., 1986; Nunzi and Franzini-Armstrong , 1981. Glaser et al., 2018.

\subsubsection{Myofibrils}

The structure of myofibrils in muscles of invertebrates is quite variable. Flight muscle of insects belong to two functional groupings: direct and indirect flight muscles (see 1.3.1). The myofibrils in the DFM of dragonfly are flat and have a very narrow width, as appropriate for a fast acting muscle (2.1.5.2 A); the myofibrils in the IFM of drosophila are cylindrical quite small and widely separated from each other (2.1.5.2 B). The fast acting muscles of a small copepod have an unusual feature: the contractile material is separated into groups (myofibrils) of very large size (2.1.5.2 C, see 1.3.2.1 A). The sarcotubular system invades the myofibrils, reducing the distance between CRUs and the contractile material. Finally the TDT of Calliphora, as most body muscles, has a more conventional division of small myofibrils by SR/T tubules (2.1.5.2 D). Glaser et al., 2018; Takekura and Franzini-Armstrong, 2002; Polyak et al., 2003.

\subsubsection{Thin/Thick Filaments}

Although both skeletal and arthropod muscles have a double hexagonal disposition of filaments in the overlap region of 
the A band, the relative position of the filaments is fundamentally different in the two muscles. In skeletal muscle thin filaments occupy the triadic position (at the center of a group of three thick filaments (2.1.5.3 A), while in arthropods they are in the dyadic position, equally distant from two tick filaments. The dyadic positioning results in a semicrystatlline $\mathrm{A}$ band structure (2.1.5.3 B and $\mathrm{C}$ ) that is in part responsible for the orderly arrangement of cross bridges in rigor muscles (see 2.1.3.5 B). The disposition in the fast dragonfly flight muscle (2.1.5.3 D) is similar, but not as precise as in Drosophia IFM. Slower fibers have longer thin and thick filaments and perhaps in order to make up for the thin filament fragility the all have a higher thin/filament ratio, up to a precise disposition of up to 24 thin filament around each thick one (2153 E and F, crab). Franzini-Armstrong, 1970a; Glaser et al., 2018.

\subsubsection{Lower Invertebrate Muscles}

The body muscles of Mollusca and worms are composed of thick filaments with a core of paramyosin surrounded by myosin, and numerous actin filaments (2.16 A). The thick filaments are longer and more variable in length than those in vertebrates and the sarcomeric structure is variable. In the fast adductor muscle of scallop the muscle is cross striated, but with a slightly irregular length of filaments, no $\mathrm{M}$ lines and irregular $\mathrm{Z}$ lines (2.1.6 B). The body muscles of earthworm, the round worm C.elegans and squid are obliquely striated, that is the banding is produced by $Z$ lines that are not perpendicular to the filaments (2.1.6 C-J). The appearance of the banding is quite different depending on the orientation of the section plane relative to the myofibrils. Thin filaments' length is proportional to that of thick filaments, but the thin/thick ratio is quite variable. The striation is most ordered in fibers that have a central core, usually containing mitochondria, and a peripheral ring of myofibrils. The sarcoplasm reticulum is located at the $Z$ lines ( 2.1.6 J), $T$ tubules are mostly missing and peripheral couplings are frequent. Nunzi and Franzini-Armstrong, 1981; Hong et al., 2001; Burkeen et al., 2004.

\subsection{Structural Cross Linkers, $\mathrm{Z}$ and $\mathrm{M}$ lines}

\subsubsection{Simple Vertebrate Z Lines}

The $Z$ line provides anchorage to the thin filaments of adjacent sarcomere allowing transmission of tension along the myofibril. In skeletal muscle the anchorage points are organized in an orthogonal array, the patterns from two adjacent sarcomere being shifted relative to each other by a half period in two orthogonal directions. The connection between the two sides are provided by obliquely arranged strands of alpha-actinin.

In the simplest $Z$ line (2.2.1 A, guppy) the actin filaments from the two adjacent sarcomeres terminate at the edges of the $Z$ line and the alpha actinin links between them form a simple zig-zag. In cross sections the patterns from adjacent sarcomere overlap in the the section and the alpha-actinin links are clearly visible (2.2.1 B and, C, small fish and toadfish swimbladder). The disposition of thin filaments changes from a random pattern in most of the I band to a precise orthogonal array in proximity of the $Z$ line (2.2.1. D toadfish swimbladder; 2.2.1. E frog leg muscle). Franzini-Armstrong and Porter, 1964b; Franzini-Armstrong, 1973.

\subsubsection{Complex Vertebrate $Z$ Lines}

Complex $Z$ lines are generated by allowing the thin filaments from the two apposed sarcomeres to overlap within the $Z$ line, where they are connected by two or more layers of alpha actinin links. 2.2.2 A is a longitudinal section of doubletiered $Z$ line from the frog sartorius. In cross sections the orthogonal pattern of thin filaments is clearly visible, and the $Z$ 
line internal structure appears as a woven pattern due the superimposition of multiple layers and the complicated path of the alpha actinin links (2.2.2 B, frog; C mouse). Variable extents of thin filaments overlap allows the construction of wider $Z$ lines (2.2.2.D mouse soleus; $E$ rabbit myocardium). The general rule is that the wider, and thus presumably stronger, $Z$ lines are in fibers that hold tension for longer periods of time, such as the fibers from the postural soles muscle and from the continuously active cardiac muscle (see 2.7.2.4). Direct comparison of $Z$ lines in fast, phasic versus slow postural muscle fibers supports the concept (222 F, mouse, $\mathrm{G}, \mathrm{H}$ frog tonic). The very extensive $\mathrm{Z}$ lines in muscle fibers from the sonic muscle of the midhshipman swimbladder (2.2.2 I) are unique and may be due to reasons other than the very prolonged love song of which this muscle is capable. See 1.4.7.4 F; 1.4.8.7 F; 1.4.3.3. B.

\subsubsection{Invertebrate Z Lines}

The filament pattern of indirect flight muscle is semicrystalline (2.2.3 A, drosophila). The regular dyadic positioning of thin filaments is maintained in proximity of the $Z$ line (2.2.3 B. Drosophila) and the $Z$ band itself is based on a hexagonal symmetry (2.2.3 C). By contrast, the $Z$ line of most other arthropod muscles is amorphous and shows no evidence for a preferred position of thin filaments in or near the $Z$ line (2.2.3 D, crab). In muscles of lower vertebrates as well as in smooth muscles of vertebrates the $Z$ band is also apparently amorphous and it appears only as a small site where thin filaments are held together (2.2.3 E, earthworm). See 1.4.10 C; 1.2.2.D; 1.2.1 F. Nunzi and Franzini-Armstrong, 1981.

\subsubsection{Line (band)}

The $\mathrm{M}$ band (or $\mathrm{M}$ line) is a structure of skeletal muscle sarcomeres visible as an extra density in the very center of the $\mathrm{A}$ band.(2.2.4 A frog). It consists of cross linkers that connect the bridge-free regions of thick filaments and hold them in a rigid hexagonal arrangement (2.2.4 B frog, $\mathrm{C}$ guppy, D toadfish swimbladder). The cross links are arranged in 2-3 tiers (2.2.4 E deep etch glass fish) and are best visible in deep etch images (2.2.4 F,G glass fish).

The $\mathrm{M}$ band bridges are absent from sarcomeres of skeletal muscle tonic fibers (as in frog tonic muscles), from vertebrate cardiac muscles and from muscles of invertebrates. Apparently the $\mathrm{M}$ bridges are not uniquely essential for the hexagonal positioning of thick elements in the $\mathrm{A}$ band which is clearly there in the absence of the $\mathrm{M}$ line. However, the $\mathrm{M}$ band gives an element of structural stability to the sarcomere. Franzini-Armstrong, 1973b; Varriano-Marston et al., 1987.

\subsection{SR and the Calcium Cycle}

\subsubsection{SR Structure}

\subsubsection{Segmented SR in Skeletal Muscle}

The sarcoplasmic reticulum (SR) is a differentiated domain of the muscle fiber endoplasmic reticulum organized in an extensive network of membranes. The SR actively takes up and stores calcium ions, thus allowing relaxation, and rapidly releases them as part of the mechanism of activation. The amount of SR varies widely depending on fiber activity (fast versus slow) muscle of origin (see 2.7.1 and 2.7.2).

The SR associates with plasmalemma/transverse (T) tubules to form Calcium Release Units (CRUs see 2.5.1) and with $T$ tubules and itself to form Calcium Entry Units (CEUs see 2.10.2).

The SR network separates and surrounds the myofibrils. In twitch fibers of skeletal muscle the network is divided into segments (one or two per sarcomere), by the presence of T tubule networks (see 2.4.2) and triads that cut across the fiber either at the $\mathrm{Z}$ line level (one/sarcomere) or at the A-I junctional level (two/ sarcomere). Hence the SR network constitutes 
either a single palisade opposite the entire sarcomere or two almost completely separate palisades, one oppose the A band and one opposite the I-Z-I segment. The A band segment is in a single layer (2.3.1.1 A, frog) with a frequency of SR element that reflects the fiber properties, more abundant in fast twitch fibers $(2.3 .1 .1 \mathrm{~B}$, frog) than in slow twitch fibers (2.3.1.1 C, frog). Aligned comparison of 1 versus 2 segment SR shows the relationship between the two alternative possibilities (2.3.1.1 D).

In a super fast muscle (toadfish swimbladder) triads are at the A-I junction and the SR is in two extensive networks (2.3.1.1 E , F). In fibers from mouse EDL a single-layer A band SR alternates with multiple-layers I band SR mixed with mitochondria $(2.3 .1 .1 \mathrm{G}, \mathrm{H})$. T tubules and triads form the segments' boundaries. Artificial coloring emphasizes the two SR segments and the position of triads, $T$ tubules and mitochondria in a mouse fiber (2.3.1.1 I). An occasional bypass of the interruption at the triads allows continuity of the SR network along the fiber.

The uniqueness of the SR segment associated with the I-Z-I region is indicated by two observations. One is she fact that calcium entry units (CEUs, see 2.10.2) ) are preferentially formed only in this segment and the second is the fact that the accumulations of calsequestrin in the lumen that mark aging are initiated at the I band level (see 4.1.1 A, B). FranziniArmstrong,, 1964, 1980, 2004a; Porter, and Franzini-Armstrong, 1965; Franzini-Armstrong and Engel, 2012; Boncompagni et al., 2010, 2012a and b.

\subsubsection{Continuous SR in Cardiac and Invertebrate Muscle}

The free SR of vertebrate cardiac myocytes and of invertebrate body muscles is organized in extensive fenestrated networks that may extend over long distances without interruptions. In vertebrate cardiac myocytes, the $T$ tubules and dyads are at the $Z$ lines, but their presence does not interrupt the continuity of the network (2.3.1.2 A-C). Continuity of the cardiac SR network is best seen in scanning electron micrographs of mouse cardiac muscle (2.3.1.2 D, E). In most body muscles of invertebrates, T-SR junctions are un the form of dyads with a prevalent longitudinal orientation, Thus, as in cardiac myocytes, the SR longitudinal continuity is not interrupted: the free SR simply bypasses the dyads (2.3.1.2 F,G). Franzini-Armstrong , 1974; Franzini-Armstrong, and Peachey, 1982; Eastwood et al, 1982; Franzini-Armstrong et al., 1986; Takekura and Franzini-Armstrong, 2002.

\subsection{2 $\mathrm{SR}$ as a $\mathrm{Ca}^{2+}$ Store}

Differential centrifugation is used to separate the membrane components of skeletal muscle homogenate into several fractions. The lightest fraction is composed of membranes derived from $T$ tubules and plasmalemma. The light fraction of the "relaxing" factor is composed of membranes derived from free SR (2.3.2 A), while the heavy fraction contains junctional SR segments (2.3.2 B). The SR vesicles actively pump calcium into the lumen, thereby reducing the cytoplasmic calcium concentration to very low levels and inducing relaxation. The relaxing function of SR is greatly enhanced by the presence of luminal calcium binding proteins, mostly calsequestrin, in the SR lumen that increase the calcium capacity (see 2.5.2.3.1 A, B). Addition of sodium oxalate to skinned fibers induces precipitate of insoluble calcium oxalate in the SR lumen (2.3.2 C) thereby confirming the SR function in situ and increasing pump capacity. Pumping is due to the insertion of the calcium activated calcium pump protein in the free SR membrane. The heads of the pump project into the cytoplasmic surface and are visible in isolated SR vesicles either after tannic acid enhancement or in freeze-dried deep etched images (2.3.2 D), see also 2.3.3. Campbell et al., 1980; Ferguson et al.,1985; Franzini- 
Armstrong, and Ferguson, 1985; Franzini-Armstrong, 1998.

\subsubsection{SR ATPase}

The calcium pump ATPase molecules appear as small "particles"on the cytoplasmic surface of freeze-fractured skeletal muscle SR membranes in situ (2.3.3 A, B). The density of particles is uniform over the entire surface of free SR, including the lateral surfaces of the SR triads, but the ATPase is excluded from the junctional SR. The particle density is quite high throughout, but in slower fibers small patches of particle-free membranes are present thus reducing the overall density. Comparison of unidirectional and rotary shadowed images shows that the freeze-fracture particles are quite variable in size suggesting grouping of the molecules into small agglomerates of variables sizes (2.3.3 C). Indeed, rotary shadows of the free surface of isolated SR vesicles show a uniform disposition of smaller particles with a density of 30,000/ $\mu \mathrm{m} 2$ (2.3.3.D, upper half). These are identified as heads of the individual ATPase molecules by comparison with the free surface of SR tubes in which the ATPase is induced to aggregate into a semicrystalline array of double molecule stripes by blocking the ATPase activity with vanadate (2.3.3 D, lower half). A comparison of free surfaces with freezefractures membranes confirms that the freeze fracture particles comprise variable counts (1-4) of individual molecules. In the free SR of most skeletal muscle the ATPase molecules are evenly distributed indicating that in the native configuration the molecules are equally distributed in all free SR (2.3.3 E, left). In the fast adductor of scallop muscle the ATPase of in situ SR has an unusual semi-crystalline helical arrangement (2.3.3 E, right), such as artificially induced in isolated SR vesicles (compare with 2.3.3D).

Free SR in body muscle of invertebrates is also rich in ATPase. But often a lower density than in skeletal muscle. FranziniArmstrong, 1974, 1984, 2004; Franzini-Armstrong and Ferguson, 1985; Ferguson et al., 1985; Franzini-Armstrong et al., 1988; Castellani et al, 1989.

\subsubsection{SR as a Calcium Source}

The majority of the SR surface, called the free SR (fSR), participates in calcium uptake due to the calcium pump protein. A smaller portion the membrane, called junctional SR (jSR) functions as the site for calcium release. jSR elements are part of the junctions with plasmalemma/ T tubules (triads, dyads and peripheral couplings) or calcium release units (CRUs). The first structural hint of CRUs' importance came from the observation that the junctional gap is occupied by large, evenly spaced densities, the feet (2.3.4 A frog, B toadfish). The same densities are present on the surface of isolated heavy SR vesicles in correspondence of calsequestrin, indicating that they are intrinsic features of the junctional SR membrane (2.3.4 C, see 2.3.2 B). The feet are the large cytoplasmic domains of ryanodine receptors, the SR calcium release channels (see 2.5.2.1). The intramembrane domains of the channels are detectable in freeze-fracture images of the junctional SR membranes as barely visible flat "particles" in double rows (2.3.4 D, toadfish, see also 2.5.3.1 D). The appearance of feet structure is variable in very thin sections, where they are represented either by two closely spaced pillars, or by single density or by a line crossing the junction (2.2.4 $\mathrm{D}$ toadfish). Feet are present in all muscles indicating that they are an early component in the differentiation of muscle (2.4.4 F, dragon fly, an insect; 2.3.4 G scallop, a mollusc). Franzini-Armstrong, 1970c; Campbell et al., 1980; Franzini-Armstrong and Nunzi, 1983; Ferguson et al., 1984; Block et al., 1988. 


\subsection{The T Tubules}

\subsubsection{T Tubule Openings}

Transverse tubules are invaginations of the plasmalemma that penetrate into the depth of muscle fibers. Their membrane is continuous with the plasmalemma and carries the electrical signal from fiber surface to interior. In most muscles the T tubules form a complex network (the T system) that surrounds and separates the myofibrils. The tubules' lumen is patent, as indicated by the free diffusion into it of extracellular traces (e.g. ferritin for electron microscopy and various fluorescent molecules for light microscopy). The orientation is not always transverse (particularly during differentiation, in pathological conditions and in some invertebrate muscles ) and association with SR is variable, in triads and dyads.

Openings of the T tubules at the fiber's edge are quite variable. A direct, wide opening is rarely seen in skeletal muscle (2.4.1 A, B, guppy). Most common is a fairly tortuous final T tubule segment and a very narrow opening (2.4.1 C, D, frog). In most skeletal muscles the T tubule openings are the same size as the openings of Caveolae and often the T tubules open in the back of caveolae (2.4.1 E, frog). An unusual disposition is found in some fish muscles. In these Golgi stain (see 5.2.1) shows $T$ tubules converging in peripheral manifolds, from which few convoluted tubules connect to the plasmalemma (2.4.1 F, toadfish; $\mathrm{G}$, small fish; $\mathrm{N}$ as seen by Veratti, 1902). T tubule openings are quite wide in cardiac muscle $(2.4 .1 \mathrm{H}-\mathrm{J}$, rabbit myocardium). The openings are also patently open in arthropod muscles and they were first described in flight muscle of dragonfly (2.4.1.K dragonfly). Scorpion muscle has an unusual feature: the $T$ tubules invagination are in the form of small longitudinally oriented slits $(2.4 .1 \mathrm{~L})$, which penetrate into the fiber $(2.4 .1 \mathrm{M})$. Emilio Veratti in 1902 illustrated the unusual arrangement of T tubules in fish muscle (2.4.1 N). Franzini-Armstrong and Porter, 1964; Franzini-Armstrong et al., 1973, 1975, 1986; Takekura et al, 2001; Franzini-Armstrong, 2002.

\subsubsection{Skeletal Muscle Transverse T Networks}

In skeletal muscle the $\mathrm{T}$ tubules are components of transverse networks that pervade the entire fiber at periodic intervals and closely surround the myofibrils without interruptions. The size and configuration of the networks are determined by the size and shape of the myofibrils (2.4.2 A, B rat; $\mathrm{C}$ frog). In fish muscle the myofibrils tend to be ribbon like in shape, the T tubules are aligned in the narrow space between the myofibrils (2.4.2 D,E toadfish) and transverse connections complete the network (2.4.2 F, toadfish). The transverse networks of $T$ tubules are either at the $Z$ lines level (in fibers with single SR segments per sarcomere) or at the A-I junctions (in fibers with two SR segments per sarcomere, see 2.3.1.1. Golgi stain reveals the double network layer at the edges of the A-I junction in rat but not in frog (2.4.2 G).

The Golgi "stain" allows direct visualization of the network by filling the lumen of the T tubules with an electron dense precipitate and showing two components of the network: wider domains and thinner tubules. The wider domains are part of triads were jSR and jT tubules are joined by double rows of feet; the short T segment between the triads are free $T$ tubules (2.4.2 H). The complete T networks were illustrated by Emilio Veratti in 1902, with an accuracy that made the newer EM images almost obsolete (2.4.2 I). Transverse networks of tubules may be either one or two per sarcomere (2.4.2 J) again illustrated by Veratti's drawings, top and by EM, bottom. Franzini-Armstrong and Peachey, 1982; Franzini-Armstrong et al., 1987b, 1991; Franzini-Armstrong 2002.

\subsubsection{Skeletal Muscle Longitudinal T Extensions}

Although with a predominant transverse orientation, the T tubule networks of skeletal muscle also have infrequent 
longitudinal extensions This may in part be a vestige of the initial development of the tubules, when the T tubule are almost completely longitudinal (2.4.3 A, see 2.11.2 A). In adult muscle short longitudinal extensions connect networks across either the A band of the I-Z-I segment (2.4.3 B, C, D guinea pig). Longitudinal extension spanning the distance between networks are particularly frequent at sites of vernier displacement of the cross striation (2.4.3 E guinea pig), where they accompany a similar disorder of the SR (2.4.3 F guinea pig). Short longitudinal T tubule extensions within the I band are essential in the formation of CEU (see 2.10.2). Flucher et al., 1993; Takekura et al., 1994; Franzini-Armstrong 2002.

\subsubsection{Junctional and Free T Tubule Segments}

T tubule networks comprise two types of segments (free and junctional) that alternate with each other. In skeletal muscle the junctional segments $(\mathrm{j} T)$ are flat, ribbon like, straight and are associated with rows of junctional feet (2.4.4 A). Thus the flat segments constitute the central element of triads. The free $T$ tubules (fT) are narrow, with approximately round cross sections and a meandering course. They are interposed between CRUs in the T network (2.4.4 A), and they also constitute the longitudinal extension oft the network (see 2.4.3). Fast fibers have a predominance of long jSR segments (2.4.4 B) that can be quite extensive. (2.4.4 D), while slower fibers have shorter jSR segments. Longer and/or more frequent fT tubules are interposed between jT segments in fibers with shorter jSR (2.4.4 C).

Using In Golgi "stained" fibers to study the contributions of $\mathrm{j} T$ and $\mathrm{fT}$ to the $\mathrm{T}$ tubule network established the fact that the ratio of $\mathrm{jT}$ to $\mathrm{fT}$ lengths is higher in faster fibers, while the overall network may be the same. This of course means that CRUs are more frequent and/or more extensive in the fast fibers. Slow tonic fibers, where the fT largely predominates, offer an extreme example (2.4.4 E). The super-fast sonic muscle in the toadfish swim bladder constitute apparent exemptions to the above rule, since in both female and male free segments are very frequent $(2.4 .4 \mathrm{~F})$. The basis for this apparent discrepancy was revealed by the observation that fT segments have a role in allowing extension of the T network when the fibers contract and the transverse network is stretched. The swim bladder inflates during swinging thus largely affecting muscle length. On the contrary, muscle fibers from the tail muscle of some fish undergo minimal length changes as the fish swims and thus have basically no fT segments $(2.4 .4 \mathrm{G}, \mathrm{H})$. Other muscles in the same subjects do show the typical fT-jT alternations (2.4.4 I). Appelt et al. 1989, 1991; Franzini-Armstrong et al., 1987, 1988; Lavorato et al., 2019.

\subsubsection{Membrane Folds, jT, fT in Arthropod Muscles}

Shape and distribution of $\mathrm{T}$ tubules are more varied in body muscles of invertebrates than in skeletal muscle. Some specializations are related to the development of large muscle fibers that require an additional structure for the inward transmission of the electrical signal. This is achieved by two strategies present in muscles of Crustacea and Polchidae: the presence of large membrane folds that essentially separate the large mass of myofibrils into subdivisions (2.4.5 A-C) and the presence in some muscles of supernumerary tubules that do not participate directly in e-c coupling but provide a an additional electrical connection between $\mathrm{T}$ tubes and the surface membrane (2.4.5 D). A second variation is in presence of T tubule networks that are not limited to the transverse planes, but use all three dimensions: an obvious example is the $T$ network of the fast acting adductor muscle of crayfish (2.4.5 E, F). An unusual organization in the fast fibers of a copepod is the presence of frequent longitudinal T tubules extensions (2.4.5 G. H) and the infiltration of of T tubules (and dyads) within very large myofibrils (2.4.5 I). Finally, one basic difference between $T$ tubules/SR relationship of arthropod body muscles and of skeletal muscle is the longitudinal rather than transverse orientation of the T/SR junctions, in the former, mostly dyads, accompanied in some cases with projections of the T tubule allowing dyad formation above and below the 
T tubule network plane $(2.4 .5 \mathrm{~J})$. A somewhat similar geometry is also encountered in a primitive fish $(2.4 .5 \mathrm{~K})$, where dyads are longitudinally oriented and the SR network is not segmented (see 2.5.1.2). Franzini-Armstrong 1974; Eastwood et al, 1982; Franzini-Armstrong et al., 1986; Glaser et al., 2018.

\subsection{E-C Coupling CRUs}

\subsubsection{Variety of CRUs}

\subsubsection{Triads}

Triads are the common organization of CRUs in adult skeletal muscle. They are composed of three elements (hence the name): a central T tubule belonging to the T network and two SR elements (2.5.1.1 A). The membranes of the three elements are connected to each other by rows of evenly spaced entities (feet) identified as the calcium release channels (RyRs). The position of the T tubule network determines where triads are located. Frog muscles have one triad/sarcomere at the $Z$ lines (2.5.1.1 A). Fast toadfish swimbladder muscles have two triads/sarcomere and section that bisect the triad show two rows of feet on either side the $T$ tubules $(2.5 .1 .1 \mathrm{~B}, \mathrm{C})$. The junctional gap between $\mathrm{T}$ and $\mathrm{SR}$ is accessible to fairly large molecules (such as ferritin), thus showing a path for free diffusion of calcium from release sites (feet or RyRs) to cytoplasms (2.5.1.1 D).

During differentiation triads have an initial longitudinal orientation but gradually rotate to a transverse orientation (2.5.1.1 E, prenatal mouse diaphragm, see 2.11.4). Franzini-Armstrong 1970c, 1971 1972; Franzini-Armstrong and Nunzi, 1983.

\subsubsection{Dyads}

Dyads, a type of CRUs, are formed by the apposition of a single SR element to a junctional segment of T tubule network, with the intercalation of feet. Dyads are present as temporary components in differentiating and pathological skeletal muscle and as constitutive elements of some slow tonic fibers, of vertebrate cardiac muscles and of most invertebrate body muscles.

In vertebrate myocardium a single SR element wraps itself around a $T$ tubule segment, sometimes almost completely encircling it (2.5.1.2 A, B, C). In thin sections two segments of a single SR apposed to the same T tubule may appear as separate elements, giving the false impression of a triad arrangement. Conversely, jSR cisternae distinguished by the condensed content of calsequestrin are often quite extensive and may connect two adjacent segments of profiles (2.5.1.2 $\mathrm{D}, \mathrm{E})$ that may be part of a single tubule (2.5.1.2 F).

Dyads are common components of body muscles of invertebrate. They differ from the cardiac dyads in that the two components (one jSR and one jT) are both flat. The SR runs along one side of the T tubules between them and the myofibrils. The noticeable features of dyads in these muscles are the extensive plaques of feet-bearing junctional surfaces that are oriented longitudinally (2.5.1.2 G-I). An unusual conformation of T tubules in scorpion muscle results in the formation of dyads at two neighboring site on either side of the $T$ tubule profile $(2.5 .1 .2 \mathrm{H})$. The presence of dyads and $T$ tubules does not result in a segmentation of the SR network of the type seen in skeletal muscle, see 1.4.8.6 A, E. Dyads may be found in close spatial relationship with mitochondria facilitating ready pick up by mitochondria of calcium released from the SR (2.5.1.2 I, J). Continuity between $\mathrm{jSR}$ and $\mathrm{fSR}$ in cardiac muscle is restricted (2.5.1.2 K). FranziniArmstrong 1964; Franzini-Armstrong et al., 1986, 1999; Takekura and Franzini-Armstrong 2002; Brochet et al., 2005. 


\subsubsection{Peripheral Couplings (PCs)}

Peripheral couplings (PCs) are associations of peripherally located jSR elements with the plasmalemma (2.5.1.3 A). PCs share function and molecular composition (RyRs and DHPRs) with triads and dyads, so they belong to the category of CRUs. PCs are the first type of CRUs to appear in differentiating skeletal and cardiac muscle (2.5.1.3 B) and are also present in cultured myotubes (2.5.1.3 C) and in sinoatrial cells that lack T tubules (2.5.1.3 D). However, PCs are virtually absent in adult skeletal muscle with few exceptions. On the contrary, PCs are frequent in most adult cardiac myocytes (2.5.1.3 E), where they coincide with dyads and extended jSR. PCs are the only type of CRU in frog myocardium (see 1.4.8.3I) and in body muscles in primitive organisms (2.5.1.3 F, C. elegans; G, scallop) see also 122 F. Protasi et al., 1996; 1997; Franzini-Armstrong et al., 1999; Tijskens et al., 2003; Perni et al., 2012.

\subsubsection{Corbular and EJSR}

In most muscles the jSR membrane bearing feet is obligatorily associated either with $\mathrm{T}$ tubules or plasmalemma membranes bearing DHPRs. Two exceptions to this rule are found in muscles of some arthropods and in cardiac muscle of vertebrates. Exception 1: dyads in the body muscles of spiders are formed by wide jSR cisternae bearing arrays of feet that are associated with smaller jT tubule membranes. Some part of the jSR membrane, as much as half of the surface, does not directly face T tubules (2.5.1.4 A and B) so that a large proportion of feet do not actually correspond to DHPR groups. Exception 2: vertebrate cardiac myocytes contain unusual domains of the SR that have feet on their surface and a reduced content of calsequestrin in the lumen, but are not associated either with the plasmalemma (in PCs) or with T tubules (in dyads). The smallest of such domains, called corbular SR, are constituted of small approximately spherical SR domains (2.5.1.4 C rabbit atrium). Corbular SR elements coexist with dyads in ventricular myocytes of mammalian heart, but are more frequent in atrial myocytes and in chicken myocardium (2.5.1.4 D,E) where T tubules and dyads are either absent or scarce. Exception 3: An extensive variation of junctional SR associated with feet, but not with plasmalemma/T tubules (the EJSR) is present in the fast beating myocardium of the finch (2.5.1.4 F). In this muscle very frequent peripheral couplings coexist with very extensive and frequent junctional SR elements that pervade the fiber cross section at the $Z$ line level (2.5.1.4 G, H). The elements were named Extended Junctional SR (EJSR) by their discover Joachim R. Sommer. Combination of PCs and EJSR permits fast activation through the cross section of the relative small myocytes, most likely by calcium activated calcium release, without need of T tubules. Bossen and Sommer 1984; Perni et al, 1984.

\subsubsection{Structural Components of CRUs}

\subsubsection{Feet and RyR}

The junctional gap between SR and T tubules/plasmalemma is filled by evenly spaced densities, the feet (2.5.2.1 A and B, frog, toadfish). In triads of skeletal muscle the feet are arranged into 2-3 parallel rows (2.5.2.1 C toadfish). Detailed images show that the feet have an approximately square profile and abut corner to corner in precise arrays (2.5.2.1 D, E fish).

The "heavy" fraction of microsomes from the muscle homogenate is composed of vesicles derived from the jSR. They bear feet on their surface in correspondence of the luminal calsequestrin (2.5.2.1 F, G from rabbit). This demonstrates that feet are intrinsic components of the jSR membrane. Shadowed images of heavy SR vesicles demonstrate the tetrameric structure of feet in muscles of invertebrates and vertebrates (2.5.2.1 $\mathrm{H}$ guinea pig, I scorpion). Purified ryanodine receptors (RyRs) identified as the SR calcium release channels are tetrameric and have two domains, a larger 
(the foot) and a smaller (the intramembrane channel) (2.5.2.1 J, K, rabbit. Comparison between isolated and in situ feet establishes the identity of feet as RyRs (2.5.2.1 L, M). Ultra thin section of toadfish muscle confirms the tetrameric structure of feet (2.5.2.1 N). Location of RyRs at the sites where EM shows triads is confirmed by immunolabeling. RyR labeled triads are located at $Z$ lines in zebrafish (2.5.2.1 O) and at two sites/sarcomere in mouse (2.5.2.1 P, Q). Franzini-Armstrong 1970; Campbell et al., 1980; Ferguson et al., 1984.

\subsubsection{Tetrads and DHPRs}

A voltage dependent calcium channel of plasmalemma and transverse tubules $\mathrm{CaV} 1$, also named the dihydropyridine receptor (DHPR) is identified as the component responsible for initiating excitation-contraction coupling.

DHPRs are not visible in thin sections, but they are exposed by the technique of freeze fracture (see 5.4.1). In fractured membranes DHPRs appear as relatively large and tall "particles" mostly partitioned in the cytoplasmic leaflet. In membranes of skeletal muscle, DHPR particles are grouped into tetrads (groups of four particles at the corners of squares) disposed in orderly arrangements (2.5.2.2 A, B toadfish). Identification of tetrads with DHPRs is based on the observation that in dysgenic muscles, null for the alpha1 subunit of DHPRs, tetrads are missing and that they are rescued by DHPR expression (2.5.2.2 C). BC3H1 is a cell line that expresses major proteins of CRUs, DHPR and RyR, and has frequent large PCs (2.5.2.2 D). The PCs are related to numerous large plaques of DHPR tetrads in various stages of completion in the cytoplasmic leaflet of the plasmalemma (2.5.2.2 E, F). Positioning a dot in the center of tetrads emphasizes their arrangement (2.5.2.2 G) and comparison between the rows of tetrads in a T tubule and the groups of tetrads in a plaque under the same alignment shows that both dispositions are based on an approximate corner to corner positioning (2.5.2.2 H). Muscle myotomes in zebrafish larvae also have PC associated with plaques of tetrads of various sizes (2.5.2.2 I, J, K) clearly showing he same disposition of tetrads as the T tubules of toadfish. Franzini-Armstrong and Nunzi 1983; FranziniArmstrong 1983,1984, 1999; Leung et al., 1988; Block et al., 1988; McPherson et al., 1991; Takekura et al., 1994b; Protasi et al., 1997; Gach et al., 2008.

\subsubsection{Calsequestrin}

\subsection{Calsequestrin Identification}

Calsequestrin (CASQ) was initially identified as the electron dense content of jSR in heavy SR fractions of the muscle homogenate (2.5.2.3.1 A from rabbit). The small molecules aggregate into long linear polymers that are crowded into the SR lumen, where they appear as a can of worms in standard thin sections (2.5.2.3.1 B, C frog), as a complex network in deep etch images (2.5.2.3.1 $\mathrm{D}$ toadfish) and as a fine meshwork in very thin sections (2.5.2.3.1 E, frog). In the CASQ rich muscle of the reptile Nerodia the network is complex (2.5.2.3.1 F) and detailed views show branching points (2.5.2.3.1 G). Distribution and content of CASQ vary widely in different skeletal muscles. The protein is mostly restricted to jSR in frog and mouse muscles, but it extends to other $\mathrm{SR}$ regions particularly in reptiles (2.5.2.3.1 H, I). By contrast jSR CASQ content is less abundant in some fish muscles (2.5.2.3.1 J, zebrafish; $\mathrm{K}$ toadfish; $\mathrm{L}$ garfish). CASQ accumulation occurs is aging. In mouse this starts in the free SR of the I-Z-I regions (2.5.2.3.1 M, see 4.1.1 B). Preferred, but not exclusive, location of CASQ at triads is confirmed by immunolabeling for the protein showing doublets of spots slightly more widespread than the RyR sites and occasional positive foci opposite the A band (2.5.2.3.1 N), Since CASQ foci are precisely located the triads, they act as foils for demonstrating Vernier shifts in the cross (2.5.2.3.1 O, P). FranziniArmstrong et al., 1987a; Perni et al., 2013, 2016; Boncompagni et al., 2012b; Manno et al., 2017. 


\subsection{Structural Components of CRUs}

The jSRs of vertebrate cardiac muscle have a distinctly different structure from those of skeletal muscle. The skeletal jSR cisternae are dilated and filled with a hydrated network of CASQ polymers, while the cardiac jSR cisternae are narrow, flat and have a visible content that is clustered into small densities (2.5.2.3.2 A). The visible content of cardiac jSR cisternae was mostly due to CASQ. The cisternae are wider (and apparently empty) in CSAQ null myocardium (2.5.2.3.2 B). The arrangement in small periodic densities is due to the content of junctin and triadin, since over expression of the two proteins results in a tighter arrangement (see 2.5.2.4). The conformation for cardiac CASQ is not intrinsically different from the skeletal isoform: the two appear quite similar when the cardiac form is over expressed (2.5.2.3.2 C, D). In dragonfly and fruit fly, two representatives of insects, the jSR has a visible content and a narrow shape, but the CASQ is not as tightly arranged as invertebrate cardiac muscle (2.5.2.3.2 E,F). In muscles of lower invertebrates (2.5.2.3.2 G, leech; 2.5.2.3.2 H, scallop; $2 \cdot 5 \cdot 2 \cdot 3.2 \mathrm{I}$, squid) the $\mathrm{jR}$ is apparently empty and fairly dilated. Campbell et al, 1980; Jones et al., 1998; Franzini-Armstrong et al., 1998; Tijskens et al., 2003; Franzini-Armstrong 2009; Takekura and FranziniArmstrong 2002.

\subsection{Polymer Structure of Calsequestrin}

CASQ is in the polymerized form at the ionic composition prevalent in the SR lumen in situ. It consists of very long linear strands in which the small molecules are linked back to back and front to front. The strands are compressed together in the SR lumen and appear as a can of worms in thin sections (2.5.2.3.3 A and B). Tracing of the strands in images from thin sections seems to indicate network branching, but apparent superimposition of unconnected strands from different depths cannot be easily dismissed (2.5.2.3.3 C). The problem of superimposition does not occur in deep-etched images of freezefractures specimens. Deep etching of rotary shadowed replicas demonstrates the fundamental network of the CASQ gel in situ (2.5.2.3.3 D). At hight magnification it is clear that the polymer strands branch and merge with repeated nodal points connecting short segments of the polymer (2.5.2.3.3 E). Perni et al., 2013.

\subsubsection{Stac3}

Stac3 is the most recently discovered essential component of CRUs. It was initially identified in zebrafish via an e-c coupling defect and traced to a mutated form of Stac3 that causes a debilitating myopathy in a group of native americans (NAM). This established a role of Stac3 in e-c coupling. Genetic manipulation in zebrafish was used to depress/enhance Stac3 expression and substitute it with the NAM mutated versions. In larvae at 4 days postfertilization (pf) triads and myofibrils are well differentiated (2.5.2.4 A) and triads have the normal structure including parajunctional RyR3 (2.5.2.4 B, see 7.5.3.4 C). In the early stages, at $27 \mathrm{hrs}$ pf stac3 null mutations in the fertilize oocyte did not result in obvious changes in DHPR tetrads (2.5.2.4 D). This was traced to DHPR RNA carried by the female cytoplasm. WT 4pf larvae have well developed T tubules (2.5.2.4 E, F) with normal display of tetrads. Tetrad frequency was greatly diminished in stac3 mutants and could not be rescued by expression of the NAM gene. By contrast WT stac3 expression rescued triads in mutated T tubules and enhanced the frequency of wild type tetrads (2.5.2.4 F). Stac3 is a facilitator of DHPR expression on junctional T tubule and a most likely a direct junctional link between DHPR and RyR. Horstick et al., 2013; Linsley et al., 2017.

\subsubsection{DHPR in Cardiac and Invertebrate Muscles}

Cardiac myocytes and body muscles of invertebrates depend on calcium entry for e-c coupling and the accepted 
hypothesis is that RyRs are indirectly activated by Calcium Induced Calcium Release rather than by a direct DHPR-RyR coupling. Proximity of DHPR to RyRs is necessary, but the direct association of DHPRs with RyRs that induces the formation of tetrads is not necessary. DHPRs particles are similar in size as those forming tetrads, but although they are clustered in spots corresponding to RyR sites, they do not have a specific arrangement within the clusters. See 2.5.2.5 A, lizard; B finch, a bird; C scorpion. A sketch shows the difference between the skeletal and the cardiac/invertebrates arrangement (2.5.2.5 D). See also 2.5.3.2 and 2.5.3.3. Sun et al, 1995; Tijskens et al., 2003; Perni et al., 2012; Takekura and Franzini-Armstrong 2002.

\subsubsection{Isoform-Specific Molecular Arrangements}

\subsubsection{RyR1 and CaV1.1 in Skeletal Muscles}

RyR1 and CaV1.1 (DHPR tetrads) are the main components of skeletal muscle CRUs within which they are colocalized (2.5.3.1 A). The spatial relationship in the position of the two sets of molecules is clearly established in triads where the positions of both RyRs and DHPRs are constrained within narrow elongated jSR and jT elements. The cytoplasmic domains of RyRs are seen as "feet" in thin sections. In most triads feet are located in double rows over the surface of junctional SR membrane, but are absent from the short segments of free SR between triads (2.5.3.1 B, C). In freezefracture images of jSR's luminal leaflet intra membrane domains of RyRs appear as double rows of shallow bumps with evidence of a tetrameric structure and a small central dip. The jT tubule membrane facing the jSR is occupied by two rows of tetrads (2.5.3.1 D, E). The distance between tetrads along the rows is twice the distance between feet indicating an alternate association of tetrads with feet $(2.5 .3 .1 \mathrm{~F})$. The positioning of feet results is a skew angle between the square outline of feet and the axis of T tubules, and tetrads show a corresponding skew, indicating a precise relationship in the positioning of feet and tetrads (2.5.3.1 G), as in the diagram of $(2.5 .3 .1 \mathrm{H})$ Grouping of DHPRs into tetrads and the ordered arrays of tetrads are dependent on the interaction with RyR1. In the absence of RyR1 (dyspedic muscle) DHPRs are clustered at CRUs but are not arranged in tetrads within the clusters (2.5.3.1 I). Relationship between feet and tetrads is maintained even where both are clustered in large plaques (2.5.3.1 J). DHPRs have a unique arrangement in primitive fish and where tetrads abut side by side rather than at corners $(2.5 .3 .1 \mathrm{~K})$. Assuming the same tetrad-to-RyR relationship as in higher vertebrates, the unique tetrad clustering of primitive fish predicts an array of feet in which alternate feet assume different angles (2.5.3.1 L). Interestingly, this disposition is found in vertebrate myocardium that in general tends to favor primitive structure and function. Franzini-Armstrong and Nunzi 1983; Ferguson et al., 1984; Block et al,. 1988; Franzini-Armstrong and Kish 1995; Paolini et al., 2004 a and b; Franzini-Armstrong 2004b.

\subsubsection{RyR2 and CaV1.2 Cardiac Muscle}

The DHPR-RyR relationship is fundamentally different in cardiac and invertebrate muscles versus skeletal muscle. RyR2 are clustered in dense foci at sites corresponding to PCs (2.5.3.2 A, frog) and to dyads (2.5.3.2 B,C, mouse).

Immunolabeling shows localization of DHPR2- and RyR2- positive foci in correspondence of CRUs (2.5.3.2 D, E chick, F frog). At this level cardiac and skeletal muscles have similar intimate relationship between location of RyR and DHPR. A closer look however reveal a fundamental difference. Although in proximity of RyR sites, CaV1.2 channels are nor arranged into tetrads and the indication is that differently from skeletal muscle, cardiac muscle RyR and DHPRs are not directly linked. This has profound implications for the cardiac e-c coupling mechanism. 
Several examples illustrate this point. Myocardium of finch (a bird) has very rapid contractions and frequent PCs (see 1.4.8.2.2 C). DHPRs clusters are located in the plasmalemma at high frequency (2.5.3.2 G, H), Similarly clusters of DHPRs are located in the plasmalemma of frog atrium in correspondence to frequent RyR-DHPR foci (2.5.3.2 I). Comparison of DHPR clusters in finch and frog $(2.5 .3 .2 \mathrm{I}, \mathrm{J})$ shows the same disorders grouping of the particles within each domain. Sketch of the relationship between RyR and DHPR positions in skeletal and cardiac muscle illustrate the concept that DHPR2 are located in proximity of RyR2, but do not bear the same strict relationship as DHPR1 and RyR1 (2.5.3.2 K). Sun et al., 1995; Protasi et al., 1996; Tijskens et al., 2003; Perni petal., 2012; Franzini-Armstrong 2016.

\subsubsection{RyR3 and CaV1.2 Equivalent in Invertebrates}

Invertebrates express single isoforms of RyR and DHPR with sequence analogies to vertebrate cardiac forms. In the majority of cases RyRs are arranged in extended plaques or ordered 2D arrays covering the flattened ovoidal regions of junctional SR that face corresponding extended regions of junctional T tubules (2.5.3.3 A-C). Filtered images of a large plaque in scorpion muscle demonstrates that differently from most skeletal muscle but similar to muscles of primitive fish alternate RyRs in the array have two different orientations (2. 5.3. A). The same is true of RyRs in an insect (grasshopper, 2.5.3.3. L).

The cytoplasmic leaflets of jT elements facing jSR are occupied by DHPR particles grouped in a random disposition not directly related to the RyR symmetry $(2 \cdot 5 \cdot 3.3 \mathrm{C})$. The same is true of the discrepancy between ordered RyR grouping and random DHPR arrangements in dragonfly, tarantula and calliphora (2.5.3.3 A tarantula; B dragonfly; C scorpion; D calliphora). Clustering of DHPRs within plaques of CRUs of crustacea and molluscs is also random (2.5.3.3 J squid; $\mathrm{K}$ crayfish). Comparison of DHPR plaques in cardiac versus scallop clusters illustrates the similarities between the two (2.5.3.3 K). Note that the same DHPR grouping results from lack of RyR. Franzini-Armstrong 1974; Takekura and Franzini-Armstrong 2002; Di Biase and Franzini-Armstrong 2005.

\subsubsection{RyR3 in Skeletal Muscle}

All triads of skeletal muscle have rows of junctional feet, composed of RyR1, that connect jSR to jT membranes. The triads of some, but not all, skeletal muscles have a prominent, additional complement of "parajunctional feet, PJF" that are located on the side of the jSR that does not face T tubules. (2.5.3.4 A frog, B fish). Presence of PJF coincides with the expression of a second RyR isoform: RyR3, which is very low in adult mouse muscle and toadfish swim bladder muscle, but at a level of $50 \%$ in frog twitch fibers and in zebrafish tail (2.5.3.4 C, D). PJF are particularly prominent in the latter (2.5.3.4 E). PJF do not control DHPR positioning and frequency of tetrads (2.5.3.4 F). Freeze fracture of frog muscle shows 2-3 rows of PJF on either side of the triad, in arrays that differ from that of JF (2.5.3.4 G, H). This is summarized in a 3D sketch (2.5.3.4 I). PJF and the RYR34 inform are necessary for the production of detectable "sparks" in skeletal muscle, see 3.1.4.2.

Some arthropod dyads display a jSR membrane covered by RyRs that extends beyond the surface of jSR apposed to jT membrane (2.5.3.4 J, see 1.3.3 E; 2.5.1.2 G-I). The feet in these extended areas should be classified as PJF. There is no evidence that they are a different isoform from the JF in these species. "Sparks"have not been investigated in muscles of arthropods. Felder and Franzini-Armstrong 2002; Perni et al. 2015; Barone et al., 1998.

\subsubsection{Models}

Diagrams of $\mathrm{CRU}$ components in skeletal muscle. 2.5.3.5 A shows a generic idealized diagram of the relationship 
between T tubules, SR and myofibrils in a skeletal muscle. This is partly incorrect because it indicates an uninterrupted continuity of T tubules and triads in the fiber cross section, which is very rare and only seen in the muscles of some fish. In most muscles while T tubules form a continuous network, the triads are frequently interrupted (2.5.3.5 B). A view of the jSR as a caterpillar shows an idealized version of how DHPR tetrads in the T tubule are functionally linked to the SR feet of skeletal muscle in controlling calcium release $(2 \cdot 5.3 .5 \mathrm{C})$. The name "feet" was derived from this early sketch. All CRUs contain associated clusters of RyR and calsequestrin (2.5.3.5 D). The array of RyRs in skeletal muscle indicates a specific association between RyRs (2.5.3.5 E). Alternate groups of DHPR tetrads are associated with RyRs within the junctional arrays of skeletal muscle (2.5.3.5 F). The 3D gel of CASQ is linked to the jSR membrane by polymerized clusters of triadin and by individual junctin molecules (2.5.3.5 G).

A complete triad model shows a high density calcium pump proteins in the free SR, array of RyRs in jSR, alternate position of DHPR in jT membrane, the complex CASQ gel and its link to jSR membrane by triadin polymers and by junctin (2.5.3.5 H). Assembled with the input of Simona Boncompagni, Stefano Perni and Montserrat Samso. Franzini-Armstrong 1970, 2002; Boncompagni et al., 2012a, Lavorato et al., 2019.

\subsection{Other CRU Components}

\subsubsection{Immunostaining of CRU Components}

Immunolabeling for RyR illuminates two sets of spots/sarcomere in mouse and one spot/sarcomere in fish, corresponding to the location of triads (2.6.1 A-D). Absence of triadin does not alter the location of RyR spots in mouse (2.6.1 E). The locations of RyR positive spots coincide with dyads at $Z$ lines in mouse cardiac muscle $(2.6 .1 \mathrm{~F}, \mathrm{G})$ and with $\mathrm{PCs}$ at the surface of frog atrium myocytes $(2.6 .1 \mathrm{H})$. Double immunolabeling confirms close colocalization of RYR and DHPR spots in chick myocardium (2.6.1 I). The locations of CASQ positive spots (2.6.1.J, K); Jct spots (2.6.1 L); and $\operatorname{Tr}$ spots (2.6.1 M, N) coincide with triads in skeletal muscle. The positions of CASQ positive spots (2.6.1 O), Jct spots (2.6.1 P, Q); and Tr spots (2.6.1 R) coincide with the location of dyads in mouse cardiac muscle. Sun et al.,1995; Protasi et al.,1996; Tijskens et al.,2003; Boncompagni et al., 2012 and b.

\subsubsection{Visualization of Junctin and Triadin}

Junctin (Jct) and triadin ( $\mathrm{Tr}$ ) are two intrinsic proteins of CRUs' protein complex which occupy the SR membrane adjacent to RyRs and extend into the SR lumen to form a functional and mechanical link with calsequestrin (CASQ). The two proteins are very small and cannot be imaged individually, but thicker sections reveal periodic anchors connecting jSR membrane to CASQ (2.6.2 A) . The anchors are less clearly visible, but still present, in thinner sections (2.6.2 B, C). Freeze substitution (2.6.2 D) and deep etching (2.6.2 E-G) reveals elongated anchors in fish muscle. Given triadin's ability to self assemble into small polymers, it is logical to assume that the anchors are composed of triad polymers as suggested in $2537 \mathrm{G}, \mathrm{H}$. This is confirmed by the lack of anchors in Tr null muscles $(2.6 .2 \mathrm{H})$. Lack of Tr and Jct in skeletal muscle results also in absence of CASQ and smaller jSR cisternae (2.6.2 H, see also 2.6.2 K). Overexpression of CASQ and Jct in cardiac muscle illustrates Jct role in condensing CASQ next to membranes (2.6.2 I, J).

The structural dichotomy of skeletal versus cardiac jSR cisternae (2.6.2.L, upper row) is eliminated by overexpression of CASQ that widens the cardiac SR cisternae and fills them with a CASQ polymer (2.6.2 L, lower row). Conversely, cardiac jSR is empty in Tr/Jct null myocadium and partly dilated (2.6.2 M) due to lack of CASQ and its anchoring by the two 
smaller proteins. Takekura and Franzini-Armstrong 1999; Zhang et al., 2001; Tiiskens et al., 2003; Shen et al., 2007; Chopra et al.,2007, 2009; Boncompagni et al., 2012a.

\subsection{Fiber Types}

\subsubsection{Tonic and Twitch Fibers in Skeletal Muscle}

Muscle fibers vary widely in their performance. Within vertebrates one major functional discrimination is between tonic and twitch fibers. Tonic fibers are multi-innervated by small synaptic boutons at multiple places along the fiber; they are not capable of propagated action potentials; they have a very slow and sustained contraction; the force of contraction is graded and determined by the rate of nerve stimulation. They are used for prolonged activity where tension maintenance rather than rapidity is important (e.g., the mating amplexus in frog).

Twitch fibers, the most common type, are singly innervated at a complex neuromuscular junction; the plasmalemma and in some cases $\mathrm{T}$ tubules conduct action potentials; the fibers contract more rapidly than tonic fibers, but do not maintain a prolonged tension.

One structural characteristic of tonic fibers was noted in the light microscope literature of the 19th century: tonic fibers were described as Felderstruktur because myofibrils could not be distinguished in cross section. Twitch fibers on the other hand have a Fibrillenstruktur because the myofibrils are clearly resoled. In EM images tonic fibers have large myofibrils often incomplete separated by sarcotubular elements (2.7.1 A, frog; B boa constrictor). In twitch fibers the myobrils are small and well separated by sarcotubular system elements and mitochondria (2.7.1, C frog, D boa). Frog tonic fibers have longitudinally oriented triads dispersed along the path of T tubules (2.7.1 E). In the fin muscles of the sand dab (fish) tonic fibers $(2.7 .1 \mathrm{~F})$ and twitch fibers $(2.7 .1 \mathrm{G})$ differ in the relative extent of free versus junctional T tubules. In zebrafish larvae the slow fibers are clustered in thin laters at the edge of the tail $(2.7 .1 \mathrm{H})$. Franzini-Armstrong 1973, 1984; FranziniArmstrong et al., 1987; Perni et al.,2016; Yiping et al., 1992.

\subsubsection{Variety of Twitch Fibers}

\subsubsection{Glycogen}

Restoration of ATP used in contraction and in other energy requiring events (such as calcium pumping by the SR) involves two pathways: glycolysis and oxidative phosphorylation. Glycolysis is faster but less efficient. It is used more extensively in fibers that require fast contractions of short duration. Oxidative phosphorylation, requiring mitochondria, is more efficient, but more slowly activated and is predominant in fibers that require prolonged activity. All fibers employ both types of metabolism, but in different proportions. The required substrates are stored in the cytoplasm. Glucose, in the form of glycogen, is associated with proteins to form small clusters, the "glycogen granules" of variable size that are most abundant in liver and muscle. The enzymes for glycolysis are also located in the cytoplasm. Lipids are stored in the cytoplasm as droplets of neutral lipids surrounded by a monolayer phospholipid membrane. The size and frequency of lipid droplets are related to the predominant metabolism of a specific fiber and to the content of mitochondria. Twitch fibers are subdivided into types based on several functional characteristics. 1) Rate of contraction, mostly determined by the intrinsic properties of the expressed myosins. Rate of cross bridges cycling is the major determinant of the difference between fast twitch and slow twitch fibers. 2) Fatiguability, defined as the relative ability to maintain the twitch amplitudes in a prolonged contraction. Fatiguability is directly related to the metabolic pattern employed in 
replenishing the ATP stores. In glycolysis the direct breakdown of glycogen replenishes ATP at a fast but not effective rate. In oxidative phosphorylation breakdown of lipids and glycogen via mitochondria is more efficient but a bit slower. Fatiguability is inversely related to the oxidative/glycolytic ratio. A useful nomenclature is to call the fast glycolytic fibers (type IX, IIB in mouse and human) "white" because they are associated with the least frequency of capillaries and have the lowest content of myoglobin. These fibers are fatiguable. At the other extreme are fatigue resident "red' fibers (Type I) that are the most oxidative: high content of mitochondria and myoglobin, association with numerous capillaries, but slow myosin, determine the color. Fast oxidative-glycolytic fibers (IIA) have "fast"myosin, an intermediate content of glycolytic enzymes and of mitochondria. Their color and fatiguability are intermediate.

All muscle fibers store store glycogen in association with proteins within granules of variable size (2.7.2.1 A, scallop). Glycogen granules content varies depending on the metabolic pattern of the fiber. In adult skeletal muscle glycogen granules occupy all available spaces between the myofibrils and occasional also within the I band (2.7.2.1 B, C human; D, E frog). The distribution is often quite variable even within fibers of the same muscle (2.7.2.1 F, G, rabbit sino-atrial node cells). Embryonal muscle cells are often loaded with glycogen $(2.7 .2 .1 \mathrm{H}, \mathrm{E} 18$ rat diaphragm). The fast adductor of scallop also contains large aggregates of glycogen granules. (2.7.2.1 I). See also the indirect flight muscle of Drosophila (2.8.1 L).

Note:most electron micrographs in this archive derive from muscles that were treated in situ with uranyl acetate salts. This treatment increases contrast but render glycogen granules invisible. Thus the above descriptions are not derived from a systematic study. Lavorato et al., 2016; Castellani et al., 1989; Lavorato and Franzini-Armstrong 2017.

\subsubsection{Mitochondria and Lipids}

Mitochondria and lipids droplets, one of the sources of energy for mitochondria, are more abundant in continuously active, but not necessary fast, "red" fibers. Fibers used for faster movements of short duration are "white", poor in mitochondria. Swimming in most fish is powered by the "red" muscle in lateral bundles (2.7.2.2 A), while white muscles are used additionally for short duration burst of swimming at higher speeds (2.7.2.2 B).

Skeletal muscles have a variable complement of fiber types mostly mixed together. The rat sternomastoid is an exception, because predominantly "white" fibers (2.7.2.2 C) and red fibers, rich in mitochondria and lipid droplets (2.7.2.2 D) are mostly part of separate bundles. In mouse muscles the unique complement of mitochondria has been clearly related to three fiber types allowing their identification in electron micrographs. Type I fibers constitute $~ 50 \%$ of the postural soleus muscles in mice: they are the slowest and most "red" fibers (2.7.2.2 E). Type IIA fibers are faster and with an intermediate complement of mitochondria. They occupy about half of the soleus and are also present in the EDL (2.7.2.2 F). The fast EDL muscles contains a small complement of Type I and IIA fibers, but are mostly constituted of type IIX and IIB fibers, the fastest and least oxidative (2.7.2.2 G). Note that all mouse muscles fibers contain a much larger content of mitochondria than homologous fibers in other mammalian muscles, because mice are continuously active. The contrast between white and red fibers in rat sternomastoid is quite striking $(2.7 .2 .2 \mathrm{H}, \mathrm{I})$. The "red" color of fiber bundles containing mitochondria rich fibers is in good part due to the abundance of capillaries surrounding the fibers (2.7.2.2 J) and establishing a close contact with them (2.7.2.2 K, see 2.14.1). As is expected, myocardium, a continuously active muscle, also contains lipid droplets (2.7.2.2 M). Franzini-Armstrong and Porter 1964;; Boncompagni et al., 2009a, 2010.

\subsubsection{Golgi "Stain", JT and jSR}


The Golgi "stain” infiltrates T tubules and SR with an electron dense precipitate (see 5.2.1 "Golgi” method). Both membrane systems are outlined at high contrast in semithin $(\sim 1 / 2 \mu \mathrm{m})$ sections revealing structural details that are not obvious in standard thin sections. The entire T tubule network is outlined demonstrating its continuity (2.7.2.3 A). The jSR appears as a flat ribbon, the fSR is a narrow, tortuous tubule. Comparison of T network in fast twitch fibers (2.7.2.3 A mouse, $\mathrm{C}$ guinea pig) with slow twitch fibers in the same muscles (2.7.2.3 B mouse, $\mathrm{C}$ guinea pig) shows a considerably higher proportion of JT versus fT in fast than in slow fibers. The differences are obvious even at the light microscope level (2.1.2.3 D). The SR membranes also are more densely distributed in fast (2.7.2.3 E, frog, G, $\mathrm{H}$ guinea pig) than in twitch slow fibers (2.7.2.3 F frog, J, guinea pig ). The major variability is in the frequency of SR tubes in the A band. Fibers with intermediate properties have intermediate A band SR levels (2.7.2.3 I, guinea pig). See 1.4.5.1 J, K. Franzini-Armstrong and Peachey 1982; Franzini-Armstrong et al., 1987b; Appelt et al., 1989, 1991; Franzini-Armstrong $2002,2018$.

\subsubsection{Z Line Width}

The width of $Z$ lines, as seen in thin sections, is quite variable and it is related to the contraction properties, rate of contraction and duration of contraction activity, see section 2.2.1. 2.7.2.4 A is from a slow twitch fiber in mouse soleus. 2.7.2.4 B compares $Z$ line width in a slow twitch fiber of soleus with a fast twitch fiber of EDL. See 2.2.2.

\subsubsection{Insect Flight Muscle Dichotomy - DFM and IFM}

Insect body muscles display a striking functional dichotomy between so called synchronous (direct, DFM) and asynchronous (indirect IFM) types.

The synchronous muscles (flight muscles in some species and body muscles in all insects) follow the standard pattern of neuromuscular activation, contraction and relaxation. They have varied functional properties and related ultrastructural distribution of mitochondria, SR and T tubules. The TDT (tergal depressor of trochanter) is a powerful muscle used for the initial pre flight jump in Calliphora: a fast but seldom used muscle it has frequent CRUs and SR, but few mitochondria (2.7.3 A). By contrast a flight control muscle in the same species is continuously active during flight. It has characteristics of an active fast muscle: small myofibrils, extensive dyads and free SR and abundance of mitochondria (2.7.3 B). The flight muscle of dragonfly is of the direct type. It is capable of fast and continuous activity: it has small myofibrils; frequent CRUs and free SR; large mitochondria (2.7.3 C, see also 1.3.1.1).

Asynchronous, indirect flight muscles (IFM) are present in most insects. They are unusual in that the calcium released by a single e-c coupling event remains high for a period of time during which a series of tension oscillations based on the property of stretch activation, interaction with elastic elements of the thorax exoskeleton and the alternating contractions of antagonistic muscles, drives the wing movements. Cytoplasmic ionized calcium level remains hight due to limited pumping by the SR. Typically IFM are "fibrilllar", with discrete small myofibrils (2.7.3 D). Spaces between the myofibrils are rich in mitochondria (2.7.3 E) and dyads are frequent, but free SR is very scarce (2.7.3 F).

DFM and IFM muscles are beautifully compared in Stokes Dr. Josephson RK Power and control muscles of cicada song: structural and contractile properties. J.Comp.Physiol. 190: 299-290, 2004.

\subsection{Mitochondria}

\subsubsection{Cristae}

Mitochondria are essential cell components that act in the production of ATP through respiration. Due to their origin as cell 
guests they maintain a separate highly permeable outer membrane enclosing the functional core. The inner membrane is selectively permeable, it holds a high membrane voltage and its infoldings or cristae are the sites of the respiratory chain. DNA for some mitochondrial proteins is located in the matrix. Other proteins are synthesized in the cell cytoplasm. The components of the respiratory chain are intrinsic proteins of the cristae membrane, located into protein complexes known as F1 particles, the site of proton-gradient driven ATP synthesis (2.8.1 A). Distribution and shape of cristae are extremely variable, mostly dictated by the cells' functional requirements. The most common disposition is that of flat parallel cristae with a frequency depending on metabolic requirements (2.8.1 B Calliphora; C dragonfly flight muscle; D mouse cardiac). Under not well determined conditions the lumen of cristae is obliterated (2.8.1 E, mouse cardiac; $F$ zebrafish tail). On the contrary, the lumen of cristae is usually wide in zebrafish tail muscles $(2.8 .1 \mathrm{G})$. In mitochondria with very abundant cristae extensive swirls are seen in certain section orientations $(2.8 .1 \mathrm{H}$ mouse cardiac; I lizard cardiac). A second structural variation is that of angular cristae $(2.8 .1 \mathrm{~J}$, mouse cardiac) that increase the surface area. Liver mitochondria have unusually limited cristae $(2.8 .1 \mathrm{~K})$, the active metabolic functions of the organ being maintained by glycolysis. Indirect flight muscle of Drosophila also has unexpectedly limited pattern off cristae (2.8.1 L).

\subsubsection{Continuities}

Mitochondria appear as separate small profiles in thin sections for electron microscopy. However most mitochondria are part of large extended continuous networks. Intermitochondria continuity is limited where mitochondria are scarce (2.8.2 A, zebrafish; 2.8.2 B, frog), but it may extend to very large mitochondria (2.8.2 C, dragonfly flight muscles). Mouse skeletal muscles fibers, even those classified as "fast fatiguable" are unusually rich in mitochondria. A cross section of a type IIA fiber shows that the complex network of mitochondria in a single fiber may be part of a single or at most two extended elements (2.8.2 D). This is particularly evident in artificially colored images where the extended intermyofibrillar networks and their continuity with subsarcolemmal mitochondria are obvious (2.8.2 E). Individual mitochondria can be traced over long distances (2.8.2 F, G) both between the myofibrils and in peripheral aggregates. Mitochondrial continuities also extend in the longitudinal direction. Longitudinal extensions of single mitochondria repeatedly branch out to surround the myofibrils at consecutive levels (2.8.2 H, I, mouse). Franzini-Armstrong 2007; Franzini-Armstrong and Boncompagni 2011.

\subsubsection{Distribution}

Mitochondria content of muscle fibers is closely related to the muscle functional type. Fibers required to perform prolonged periods of activity require larger content of mitochondria and the use of oxidative phosphorylation as the dominant metabolic process Other fibers have fewer mitochondria and depends on glycolysis. Examples in this set are all from vertebrate skeletal and cardiac muscles.

The mitochondria in predominantly glycolytic frog muscle fibers are few and located in longitudinal slits between the myofibrils (2.8.3 A). In some fish muscles the mitochondria are relegated to fiber periphery and/or a central core, and are excluded from the inter myofibrillar spaces $(2.8 .3 \mathrm{~B}, \mathrm{C})$. On the contrary, in mouse fibers, both fast-twitch and slow twitch, mitochondria form an extensive network around and between the myofibrils (2.8.3 D, E), and are placed at frequent repeating intervals $(2.8 .3 \mathrm{~F}, \mathrm{G})$. Cardiac mitochondria are also intermingled with myofibrils either in single columns and or in small groups $(2.8 .3 \mathrm{H}$, mouse; I rabbit). Additional mitochondria, perhaps with specialized function, accumulate either into peripheral clumps (2.8.3 J, rat) or in a paranuclear position in cultured cardiac myocytes $(2.8 .3 \mathrm{~K}$, rat). The specific location of doublets of mitochondria at the I band in mouse EDL is clearly shown in SEM images (2.8.3 L) and confirmed in 
thin sections (2.8.3 M). Franzini-Armstrong 2007; Boncompagni et al., 2009a; Franzini-Armstrong and Boncompagni 2011.

\subsubsection{Positioning}

The activity of mitochondrial respiratory enzymes involved in oxidative phosphorylation is enhanced by a small entry of calcium into the organelle. The calcium signal from SR to mitochondria is essential for mitochondria function at sites of high ATP demand, such as in rapidly and continuously contracting muscles. The signal is not due to a direct ER/SR to mitochondria communication, but rather to the proximity of mitochondria to SR calcium release sites (CRUs). As first suggested by Tullio Pozzan. opportunistic location of mitochondria near CRUs allows the former to experience the localized calcium signal during e-c coupling. Mitochondria and CRUs occupy the same restricted intermyofibrillar spaces (2.8.4 A, frog), they are specifically located near each other s (2.8.4 B, mouse) and linked other over extended regions (2.8.4 C, D , E). A close mitochondrion-CRU relationship is obviously a requirement in cardiac muscle (2.8.4 F, G rat). Note that the mitochondrial outer surface is in close proximity to, but not in contact with, the calcium release channels $(2.8 .4 \mathrm{G}$ rabbit). Close relationship between mitochondria and CRU is true in muscles of invertebrates as well (2.8.4 I). The unusual exclusion of mitochondria from intermyofibrillar spaces and proximity to CRUs in the fast toadfish swim bladder muscle is the exact contrary of the relationship in mouse (2.8.4 I). The difference begs an explanation.

IP3Rs are located within extensive ER stacks in the Purkinje cells of mammalian cerebellum. The stacks are tethered to the surface of mitochondria, but there's no contact between IP3Rs and the outer membrane of mitochondria (2.8.4 K, see also 2.9.3 A-C). Franzini-Armstrong and Boncompagni 2011; Boncompagni et al., 2009b: Sharma et al., 2000.

\subsubsection{Kissing Junctions and Nano Tunnels}

Mitochondria require exchanges with each other to maintain their function. Evidences for mito-to-mito talk have been provided by techniques that allow selected temporal visualization of either matrix or internal membrane components and a follow up of the exchange of these component between mitochondria, extending over time and relatively large distances. Three possible mechanisms have been proposed and/or explored. Two are most likely to be exploited in myocardium, where mitochondria are clearly separate organelle and seem to be rigidly trapped between the myofibrils. Using appropriate laser activated dyes, an unusual, interesting the act of intermitochondrial communication has been detected in cultured cardiac myocytes (2.8.5 A and B). This involves the protrusion of long thin nanotubes that carry mitochondria content over fairly long distances. Nano tunnels are fairly frequent in normal cardiac myocytes (2.8.5 C, D, E), and best visualized by SEM (2.8.5 F), but are not seen in other muscles. A second communication mechanism has been postulated based on the presence of kissing junctions at sites of small close contacts between adjacent mitochondria (2.8.5 G, H). A third possibility is an intermittent process of fusion/fission whereby mitochondria maintain their individuality while exchanging their contents for some brief period of time. This is most likely to be the mechanism in skeletal muscle, where mitochondria are dynamic. The extensive, mobile mitochondria of mouse fibers (2.8.5 I) are good candidates for a fissionfusion mechanism of intermitochondria transmission. See also 2.8.6. Franzini-Armstrong 2007; Huang et al., 2013; Lavorato et al., 2017, 2020.

\subsubsection{Dynamics}

In electron micrographs of skeletal muscle the extensive transverse network of mitochondria may seem static, but in reality it is probably maintained by multiple fission and fusion events $(2.8 .6 \mathrm{~A})$. A higher order of mobility is demonstrated under conditions of stress, such as in muscles exercised to fatigue. Increased frequency of constrictions events are observed 
under these conditions. (2.8.6 B). The constrictions involve the extreme thinning of a central mitochondrial domain and are extremely suggestive of a prelude to fission $(2.8 .6 \mathrm{C})$. Frequency of extreme mitochondrial constrictions (EMCs) is higher in exercised than resting muscles (2.8.6 D). SR elements are intimately associated with constriction areas (2.8.6 E) suggesting a possible involvement, as proposed for other cells. Lavorato et al., 2018.

\subsection{IP3Rs}

\subsubsection{IP3Rs in Nuclear Envelope}

Inositol trisphospate receptors (IP3Rs) and the analogous ryanodine receptors (RyRs, see 2.5.2.1) comprise two families of ionic channels that mediate rapid release of calcium from the endoplasmic/sarcoplasmic reticulum (ER/SR) lumen into the cytoplasm. IPR3s are located in the membranes of various ER domains, including the intercalated disc and the outer nuclear envelope membranes (2.9.1 A), where they are appropriately positioned for playing a synergistic role with RyR release from CRUs. Following overexpression of IP3Rs, the cytoplasmic domains of the channels can be visualized by rotary shadowing on the exposed cytoplasmic surface of the double layered nuclear envelope (2.9.1 B). The over expressed proteins have the small square structure expected of the exposed hydrophilic domains of IP3Rs (2.9.1 C). This confirms that the channels are appropriately positioned for their function of releasing calcium into the cytoplasm. Overexpressed type 1 and 3 receptors are both located at the nuclear envelope (2.9.1 D). Cardenas et al., 2010; Escobar et al., 2011 .

\subsubsection{IP3Rs and RyRs: Calcium Microdomains}

IPRs and RyRs play a synergistic role in muscle cells by virtue of the fact that RyR release sites (CRUs) are in close proximity to the nuclear envelope carrying IP3Rs and that IP3Rs need a calcium signal to be activated. In cardiac myocytes transverse tubules and RyRs (within dyads) are present in proximity of the nucleus (2.9.2 A) and dyads are associated with nuclei (2.9.2 B). This brings sites of RyR mediated calcium release in close contact with the nucleus (2.9.2 C). Double labeling for RyRs and IP3Rs shows them in close proximity to each other at the nucleus surface (2.9.2 D). Escobar et al., 2011.

\subsubsection{IP3Rs in ER Stacks}

The Purkinje cells in the cerebellum, the major output from that brain region, have an unusual arrangement of IP3Rs. The channels are inserted at a high density in semicrystalline arrays into the membranes of ER stacks constituted of multiple lamellae. The stacks are specifically located in close proximity of mitochondria, and they seem to be linked to the organelles (2.9.3 A). This provides an opportunity for transmission of a signal from ER calcium release to calcium uptake by mitochondria by virtue of local proximity as suggested by Tullio Pozzan and Rosario Rizzuto. The association is dominant: in a count of 121 profiles of stacs and mitochondria 42 (35\%) are stacks associated with mitos; $49(40 \%)$ are mitochondria only; $30(25 \%)$ are stacks only. Considering that many of the adhesion sites are simply missed by the thin section, this indicates a very high degree of association. For clarity, the lumina of individual stack elements are colored in 2.9.3 B. IP3 receptors are visible as periodically arranged densities of the cytoplasmic surface of all stack membranes, except for the membrane that faces directly the mitochondria. The latter is associated with small tethers connecting it to the nuclear envelope $(2.9 .3 \mathrm{C})$. The conclusion is that IP3 receptors in these cells do not contact the mitochondria. In collaboration with R.V. Yier 


\subsection{SOCE}

\subsubsection{STIM/ORAI System}

Calcium homeostasis in the cell is controlled by the coordinated interaction of two key molecules: STIM1, an intrinsic component of the endoplasmic reticulum membrane and Orai1, a calcium channel of the plasmalemma. The two are responsible for the activation of the store operated calcium entry (SOCE) mechanism that restores calcium deficit in the depleted ER. Ultrastructural analysis of cells overexpressing the two key molecules, separately, in conjunction with each other and under stimulation by thapsigargin mimicking calcium depletion reveals key properties of the system. STIM makes the ER surface"sticky" so that when the protein is over expressed ER cisternae adhere to each other and to the plasmalemma (2.10.1 A, B). Under stimulation by thapsigargin, a treatment that induces SOCE, the configuration of STIM is visibly altered: the molecules are clumped as they become ready to interact with Orai1 (2.10.1 C). Overexpression of Orai1 per se does not induce SOCE and the expressed channels, even when at high concentration,, remain mostly randomly distributed in the plasmalemma $(2.10 .1 \mathrm{D})$. Overexpression of both STIM1 and Orai1 and stimulation by thapsigargin induces the activated STIM1 to trap Orai1 channels into large clustered patches and activate them, resulting in SOCE (2.10.1 E). Perni et al., 2015.

\subsubsection{Calcium Entry Units in Skeletal Muscle}

Activation of contraction in skeletal muscle has been considered for many years to be independent of extracellular calcium, so calcium depletion was not excepted to play a role in muscle physiology. However minor calcium traffic across the surface membrane/ T tubules was detected early on and more recently, the STIM1-Orai system was shown to be active even for single contractions. Prolonged activity induces the accumulation of $\mathrm{Ca}$ depletion sites and the formation of a new organelle, the calcium entry unit (CEU) in which concentric stacks of SR membranes carrying STIM1(2.1.2 A) interact with Orai1 channels of T tubule extensions (Protasi et al., 2010). SR membrane stacs are not frequent in quiescent muscle, but increase in frequency under regimen of strenuous exercise and are more frequent in aging muscle; in fibers deprived of junctin/triadin (2.10.2 B); in muscles involved in repetitive contractions as in sound emission,(2.10.2 C) and in the legs of finches (2.10.2 D). Boncompagni et al., 2010, 2017; Protasi et al., 2020.

\subsection{Differentiation of the Sarcotubular System}

\subsubsection{Birth of CRUs}

Formation and maturation of $\mathrm{CRUs}$ requires the independent expression of individual components and their assembly into a functional multimolecular complex. The two major players in e-c coupling, RyR and DHPR, are most likely to follow independent pathways between synthesis and association in CRUs, since the former is an intrinsic component of the internal endoplasmic reticulum and the latter is inserted into exterior membranes plasmalemma and transverse tubules membrane $(2.11 .1 \mathrm{~A})$. Junctophilin induced docking of $\mathrm{SR}$ to exterior membranes is an essential step in initiating $\mathrm{CRUs}$ assembly.

The sequence of events is clearly illustrated in the PC differentiation of developing chick myocardium (2.11.1 B). Small, early SR cisternae are associated with the plasmalemma, but have little structure. At more advanced stages the cisternae increase in size, feet occupy the junctional gap and the jSR content is condensed. Simultaneously DHPR plaques 
appear in the plasmalemma (2.11.1 C). The frequency and size of RyR and DHPR positive spots increase in parallel (2.11.1 D). Accrual of CASQ and Jct can be visualized in cardiac myocytes overexpressing the two proteins (2.11.1 E). In WT development (top row) CASQ is visible in jSR, it is initially dispersed and at later time condensed by Jct. The rest of the image illustrates the antagonistic effects of CASQ in spreading out the jSR content and enlarging the cisternae and of Jct in clustering the CASQ and tightening the jSR cisternae. Sun et al., 1995; Protasi et al., 1996; Flucher and FranziniArmstrong 1996; Tijskens et al., 2003b.

\subsubsection{CRU Positioning}

Differentiation of the membrane system in skeletal muscle fibers involves several steps. One is the synthesis of CRU components and their coordinated interactions in the formation of CRUs. The initial association of SR cisternae with the plasmalemma, mediated by junctophilins, precedes the formation of myofibrils and transverse tubules (2.11.2 A, see 2.11.1 for cardiac muscle).

Interaction of DHPRs with RyRs, with the formation of tetrads, as well as calsequestrin contents of the jSR are part of the initial events (2.11.2 A). The invaginations of plasma membranes tubules that eventually constitute the "transverse" tubular network starts fairly abruptly. In mouse embryos this coincides with the movement of nuclei from central to peripheral positions and the first assembly of myofibrils. The early tubules have a predominantly longitudinal orientation and show no apparent relationship to sarcomeres (2.11.2 B). As soon as the first invaginations appear CRUs move to internal positions by the association of jSR with the tubules to form triads and PCs mostly disappear. This event is very rapid in zebrafish, where caudal to rostral myotomes a few hrs post fertilization illustrate the evolution of PCs to internal triads within a few hours of developmental time (2.11.2 B, C. D, see 2.5.3.4 B).

In mouse and chick embryos the $\mathrm{T}$ tubules maintain a random orientation $(2.11 .2 \mathrm{~F})$ for several weeks. During this period triads are formed by association of jSR with longitudinal T tubules, so that the triads have longitudinal orientation (2.11.2 G). However, unexpectedly, the position of the developing CRUs is at the appropriate level relative to the myofibrils cross station starting from the earliest stages $(2.11 .2 \mathrm{H})$, indicating the formation of a link between CRUs and sarcomeres. Franzini-Armstrong 1991; Takekura et al., 1993; Flucher et al., 1993; Takekura and Franzini-Armstrong 1994; Protasi et al., 1996.

\subsubsection{T Tubules and Triads}

The images illustrate a comparison of T tubules and triads development in chick slow (ALD) and fast (PLD) muscles between E14 (in ovo) and D 25 (after hatching). T tubules were labelled with a fluorescent lipid soluble dye and imaged by confocal scanning microscopy. SR contrast for EM was enhanced by potassium ferrocyanide. T tubule differentiation follows the same trend as in mouse muscles: starting with a dominant longitudinal orientation and a slow, gradual shift to transverse positioning (2.11.3 A). Triads also start with a longitudinal orientation, but they are appropriately located relative to the sarcomere $(2.11 .3 \mathrm{~B})$ and become fully transverse several days after hatching. Final transverse orientation is achieved late in both PLD and ALD $(2.11 .3 \mathrm{C}, \mathrm{D})$. The large pectorals muscle has $\mathrm{T}$ tubules and triads at the $\mathrm{Z}$ line in the adult, but start with a double set of both in the early development. CRU (triads) density increase dramatically in the fast PLD after E18, when its rate of contraction increases (2.11.3 E). Takekura et al., 1993.

\subsubsection{Maturation}

The initial formation of the sarcotubular system is followed by growth and rearrangement while the muscle fiber sizes 
increase. In some species e.g. mouse, the final maturation is quite slow and thus it can be easily followed. Caveolae facilitate the invagination of new T tubules (2.11.4 A, B, C) see also 2.13.1. CRUs rapidly evolve into triads/dyads as soon as $\mathrm{T}$ tubules appear and they increase in size and complexity (2.11.4 D), following a scheme in which accrual of junctin/triadin, calsequestrin is followed by RyR and DHPRs (2.11.4 E). Note that while in normal development association RyRs and DHPRs with CRUs is simultaneous, experimental evidence indicate that the two molecules are independently targeted to CRUs, since the junctions are formed in the absence of either molecule or both (2.11.4 F). jSR associates with the $T$ tubules while they still have the original longitudinal orientation (see 2.11.2 C) so the axis of early triad is longitudinal and the transfer to transverse orientation is achieved gradually $(2.11 .4 \mathrm{G}-\mathrm{I})$, while theT tubules are pulled onto their mature position. Franzini-Armstrong 1991.

\subsection{Cell Junctions}

Cardiac myocytes are structurally and functionally connected to each other via a variety of cell junctions. Adhering junctions (2.12 A rabbit) connect the terminal actin filaments of myofibrils in one cell to those in the other at the intercalated disc. Gap junctions also at the intercalated disc (2.12 B rabbit, C lizard) join two adjacent cells via channels that allow exchange of ions and small molecules between the cytoplasm of adjacent cells and thus act as sites for transmission of action potentials. Combinations of gap and adhering junctions (2.12 D cat) and gap junctions and desmosomes (2.12 E cat) connect adjacent cardiac Purkinje cells mechanically and electrically side by side.

\subsection{Other Organelles}

\subsubsection{Caveolae, Aquaporins and Lysosomes}

Caveolae are fixed invaginations of the plasmalemma with small neck openings often shared by several Caveolae (2.13.1 A frog). Caveolae can be filled by an extracellular space tracers and their neck openings are visible in freeze-fractures of the plasmalemma, showing their distribution on frog twitch and slow fibers $(2.13 .1 \mathrm{~B}, \mathrm{C})$. Caveolae are very numerous in cells of the cardiac sinoatrioal node (2.13.1 D). Caveolae are fixed feature of the plasmalemma, but they mediate membrane invaginations during formation of $\mathrm{T}$ tubules and thus they are numerous during periods of intense $\mathrm{T}$ tub formation (2.13.1 E, see also 2.11.4 A-C).

Aquaporins are water permeable channels that permit osmotic regulation across membranes. They are particularly numerous in the surface membranes of astrocytes, in kidney collecting ducks and in muscle fibers. Their expression in the latter is fiber type dependent and regulated by innervation. Aquaporins aggregate into small crystals in the plasmalemma. Their are arranged in small semicrystalline arrays visible as groups off particles in the inner leaflet and pits in the outer leaflet freeze-fractured plasmalemma (2.13.1 F, G toadfish swim bladder). Lysosomes are membrane bound organelles that carry a variety of hydrolytic enzymes with an acidic $\mathrm{pH}$ optimum. The enzymes are synthesized in the rough ER and delivered by the Golgi system. The substrate comes either from the cell or from outside. Secondary lysosome, derived from the fusion of the primary Golgi-derived lysosomes are larger structures easily identified by the dense content in the process of hydrolysis (2.13.1 H mouse EDL; 2.13.1 I mouse myocardium). They are fairly rare in normal muscle but more frequent in aging. Pellegrino and Franzini 1963; Franzini-Armstrong et al., 1975; Dulhunty and Franzini-Armstrong 1975, 1977; Franzini-Armstrong 1991. 


\subsubsection{Microtubules, Golgi System, Nuclei}

Microtubules are long polymers of tubulin in the shape of hollow tubes $\sim 25 \mathrm{~nm}$ in diameter. They appear fairly rigid and unbending. They act as tracks for the movement of membrane-limited organelles and they play a major role in directing oriented organization of muscle components during development. They are quite numerous in both skeletal and cardiac muscle (2.13.2 A), as shown by the work of E. Ralston. They are hard to find in conventional thin sections (2.13.2 B), but they are emphasized in tomograms of rapid frozen tissue in relation to mitochondrial nano tunnels (2.13.2 C). See 2.8.5.

The Golgi system is a complex of membranes responsible for the maturation, packaging and transport of proteins. It is easily recognizable due to its structure of stacked membranes (2.13.2 D). At the trans region Golgi delivers small vesicles containing protein for extracellular export, structural proteins and lysosomal enzymes.

Nuclei are located at the periphery of most adult skeletal muscle fibers (2.13.2 E), but they are internal if frog muscles (2.13.2 F). The envelope is perforated by a large number of pores (2.13.2 G lizard) that have a very complex associated structures and control exchange between nucleoplasm and cytoplasm $(2.13 .2 \mathrm{H})$. IP3 receptors are components of the nuclear envelope (see 2.9.1). The nucleolus (2.13.2 I) often multiple (2.13.2 J) contains multiple copies RNA coding for ribosomal proteins. Lavorato et al., 2017.

\subsection{Muscle Tissue, Accessory Components}

\subsubsection{Capillaries and Pericytes}

Capillaries, the smallest branches of the circulatory tree, invade the muscle endomysium and are in close proximity to the muscle fibers. They are most frequent around oxidative slow twitch fibers, where they contributing to the "red" color of the muscles (2.14.1 A rat). Numerous capillaries surround individual "red" fibers $(2.14 .1 \mathrm{~B}$, rat) and are frequent in red bundles of soleus (2.14.1 C, D mouse) but less frequent in proximity of "white" fibers. Next to mitochondria- rich fibers capillaries are appropriately located in grooves of the fiber surface in close proximity of mitochondria (2.14.1 D). This establishes a close relationship between circulatory and muscle systems, although the two are separated by a thin layer of endomysium. Proximity between capillaries and mitochondria facilitates gas exchanges (2.14.1 D-G rat and mouse).

Muscle capillaries are associated with pericytes, close relatives of the smooth muscle cells associated with larger blood vessels. Branches of pericytes surround the capillary endothelium $(2.14 .1 \mathrm{H}, \mathrm{I})$. Long pericytes cover the surface of capillarities (2.14.1 I- L mouse) hugging them with fingers that are suggestive of a vasoconstriction capability. Pericytes are also present in the wall of venules (2.14.1 M). Mazanet and Franzini-Armstrong 1982a.

\subsubsection{Satellite Cells, Fibroblasts}

Satellite cells (Mauro, A. J. biophys. biochem. Cytol. 9:493-495, 1961) are present only in differentiated skeletal muscles and constitute 3 to 10 percent of the nuclei near the fiber edge. Vertebrate cardiac muscle and invertebrate body muscles do not have obvious satellite cells. Satellite cells are committed myoblasts that remain in a quiescent state, as indicated by the condensed chromation and are located under the basal lamina (2.14.2 A). They are spindle shaped with a smooth surface and are partly hidden by connective tissue (2.14.2 B). They occupy a niche in the fiber outline (2.14.2 B and C). Satellite cells respond to muscle damage (2.14.2.G) by becoming activated (2.14.2.D, E, F) detaching and proliferating to fuse and form new tissue.

Fibroblasts are cells of the connective tissue dedicated to synthesis and secretion of extracellular matrix proteins. They 
are components of the endomysium, in close proximity to the muscle fibers $(2.14 .2 \mathrm{H}$ dog heart, I cat extraocular muscle, $\mathrm{J}$ zebrafish tail muscle). They have prominent rough ER and Golgi systems. In muscle tendon fibroblast are flattened, surrounded by large bundles of collagen fibers (2.14.2.K cat extra ocular muscle). 2.14.2 L illustrates the method for preparing muscle fibers for EM. A small bundle of fixed muscle fiber was tied to a stick before treatment with OsO4 fr maceration. The connective tissue was totally extracted by maceration so the the muscle fire float freely. See 5.5.1 for the technique. Castillo de Maruenda and Franzini-Armstrong 1978; Mazanet et al, 1982b; Bischoff and Franzini-Armstrong 2004.

\subsubsection{Myotendon Junction, Spindle}

The myotendon junction provides anchor for the myofibrils at the muscle fiber's end and it transmits the tension generated by contraction to the tendon. It is particularly convoluted where skeletal muscle joins the bones. Mechanical strength is assured by the convoluted surface of the fiber ends, with deep infoldings penetrated by collagen fibers anchored at the plasmalemma. On the interior side of the junction myofibrils terminate at the $Z$ line with extensive aggregates of actin filaments, (2.14.3 A and B, frog).

Stretch receptors are associates of the muscle system that provide static and dynamic information on the muscle length. They are part of the feedback loop that allows fine control of muscle activity. In skeletal muscle the stretch receptors are located within a special domain, the muscle spindle, where different muscle fiber types and both sensory and motor nerves are contained within a space separated from the adjacent muscle fibers by multiple layers of endothelium and connective tissue (2.14.3 C mouse EDL). The term spindle is indicative of the organelle's shape: centrally wide and tapered at either end.

\subsubsection{Axons and Nerves}

Nerve axons are bundled together into nerves separated from the other tissues by a thin cell layer. In vertebrates the fasterconducting axons are myelinated: they are covered by myelin constituted of multiple layers of cell membranes laid by oligodendrocytes in central nerve and Schwann cells in peripheral nerves. The layers of cell membrane are very closely apposed constituting an effective insulation and increasing the axon rate of conduction (2.14.4 A and B). Saltatory conduction of the nerve impulse is assured by the presence of Nodes of Ranvier, where the myelin membranes become separated and ionic permeabilities are present (2.14.4 A and B). The axons contain microtubules, neurofilaments (intermediate filaments) and mitochondria alighted longitudinally along the axon.

Non myelinated axons are mostly of smaller diameter. Several axons are sheltered into deep folds of a single Scwhann cell that totally surrounds them (2.14.4 D). Small non myelinated axons are distributes in heat, smooth muscle and intestine (2.14.4 C, Finch heart), see 1.4.10 B.

Nerves of zebrafish larvae contain a mixture of myelinated axons with myelin in the process of compacting and non myelinated axons (2.14.4 D-F). Rosenberg et al., 2012, 2014; Monk et al., 2013.

\subsubsection{Neuromuscular Junction}

Neuromuscular junctions (nmj) are of two types. One type is found in most body muscle of invertebrates, in skeletal muscle fibers that do not conduct an action potentialial and in muscles in some species (see 2.7.1). These nmj junctions consist of the multiple association of individual small terminal boutons of motor axons branches with multiple regions of the muscle fiber. Synapses in the tail muscle of crayfish are good examples. Branches of the nerves follow the surface of the muscle 
fiber and have frequent active zones or sites where the transmitter is released $(2.14 .5 \mathrm{~A})$. These correspond to small patches of receptors in the postjunctional membrane that have two appearances corresponding to excitatory and inhibitory synapses $(2.14 .5, \mathrm{~B}, \mathrm{C})$. Muscle fibers of the tail myotomes of zebrafish are innervated by small nerve endings located near the myotendon junctions (2.14.5 D-F). The synaptic endings contain secretary vesicles and are separated from the post junctional membrane by a wide basal lamina.

A second type of terminal, the motor end plate, is a single complex structure located in the middle region of twitch muscle fibers capable of action potentials. It consists of a differentiated region of the postsynaptic muscle fiber forming an elevate mound with embedded nuclei and complex postynaptic folds containing acetylcholine receptors in the membrane and acetylcholine esterase in the basal lamina $(2.14 .5 \mathrm{G})$. A single axon branches into a cluster of multiple small terminals protected by a Schwann cell $(2.14 .5 \mathrm{H}, \mathrm{I})$. The terminal boutons occupy a groove of the muscle cell indented into numerous folds (2.14.5 J, K). In an elongated terminal from frog, differentiated active zones (release sites) of the nerve membrane are aligned with openings of the postsynaptic clefts (2.14.5 L, M). Mouse NMJ, Matias.Escobar, Kristen Colavita, unpublished images; Armstrong 1976; Lefebvre et al., 2004, 2007.

\section{Part 3. Experiments}

\subsection{Exploring RyR, DHPR and Stac3 Roles}

\subsubsection{DHPR in Dysgenic Muscle}

A colony of mouse carrying a recessive lethal mutation was maintained for many years with no aim in mind, until the mutation was identified by the work of J. Powell, K.G.Beam and T Tanabe as the failure to express the alpha1 subunit of DHPR's complex. In the absence of functional DHPRs the link between depolarization and contraction was missing: the dysgenic muscle fibers had normal action potentials, but did not release calcium and thus did not contract.

A direct correlation between DHPRs and the freeze-fracture particles arranged into tetrads in the membranes of plasmalemma/T tubules at CRUs (see 2.5.2.2 folder) was established by the expression of dihydropyridine receptor cDNA in cultured dysgenic myotubes. Particle tetrads that were totally absent in the mutant myotubes were restored by DHPR expression (3.1.1 A and B) that also restored the missing e-c coupling activity. Takekura et al., 1994.

\subsubsection{DHPR Tetrad Rescue}

An allosteric interaction between DHPRs and RyRs based on the link of DHPR tetrads with RyRs is an essential requirement for e-c coupling of the skeletal type, i.e., independent of extracellular calcium. The domain of skeletal muscle DHPR responsible for restoring tetrad formation and skeletal type e-c coupling was explored in dysgenic (DHPR null) myotubes by expressing cardiac-skeletal DHPR chimera. The following constructors restore (+), or fail to restore (-) tetrads and skeletal e-coupling The full alpha1 DHPR subunit (3.1.2 A+); the cardiac DHPR with skeletal II-III loop (3.1.2 B+); the cardiac alpha 1 with cardiac II-III loop (3.1.2 C-); skeletal alpha1 with cardiac II-III and S45 skeletal segment (3.1.2 D+); skeletal alpha 1 with Musca II-III (3.1.2 E, +, but negative function); skeletal alpha 1 with Musca S 5 segment (3.1.2 F +). See 3.1.2 G. Note: a duplicate of each image with tetrads "dotted" is presented. Flucher and Franzini-Armstrong, 1996; Fessenden et al., 2001; Takekura et al.,2004.

\subsubsection{Dyspedic Mutation and Rescue}

A targeted null mutation (skrrml) in mice of the gene for skeletal muscle RyRs results in the absence of e-coupling in 
homozygous offsprings of transgenic (dyspedic) parents. CRUs (dyads and peripheral couplings) are present in mutated muscles, but lack junctional feet (3.1.3 A). DHPRs are present but disorganized, demonstrating that association with feet subunits determines clustering of DHPR into tetrads (3.1.3 B, left). Feet and DHPR tetrads are rescued by expression of RyR1(3.1.3 B, right). Despite lack of feet, triadin is appropriately clustered in dyspedic CRUs (3.1.3 A). Takekura et al., 1995a; Takekura and Franzini-Armstrong, 1999.

\subsubsection{Roles of RyR1 and RyR3}

\subsubsection{In vitro Experiments}

The two RyR informs in skeletal muscle, RyR1 and RyR3, have different functions. RyR1 is the essential structural and functional component of the SR/T tubule interaction, while RyR3, where present, takes a peripheral position in the junction and a secondary role (see 2.5.3.4). When expressed in a RyR null background (dyspedic myotubes 3.1.4.1 A) RyR3 occupies the dominant position in PCs (3.1.4.1 B, C), but it fails to organize DHPRs into tetrads (3.1.4.1 D). The possible interactions between RyR1 and RyR3 and DHPR in dyspedic and WT CRUs are illustrated (3.1.4.1 E). The contribution of the two isoforms to the RyR-tetrad link and to the RyR1-DHPR reciprocal interaction were explored using a series of chimeras where segments were exchanged between RyR1 a (3.1.4.1 F). Rescue of tetrads correlates with recovery of the skeletal type specific e-c coupling, but not precisely . A broad region of the RyR1 sequence is essential for all functions, but could not be further refined. Nakai et al., 1997; Barone et al., 1998; Protasi et al., 1998a, 2000, 2002.

\subsubsection{Sparks}

The RyR3 isoform of the SR calcium release channel does not sustain e-c coupling, and is located in a parajunctional position in the triad (3.1.4.2 A, see 3.1.4.1 and 2.4.3.4). In zebra fish block of RyR3 expression at fertilization by an engineered morpholino lasts several days. Triads are fully developed at $27 \mathrm{hrs} \mathrm{pf}$. and the overall fiber structure is not altered by the morpholino action (3.1.4.2 B), but parajunctional feet are eliminated (3.1.4.2 C). The magnitude of the tail flip is also not affected (3.1.4.2 D). The only significant effect of RyR3 lack is the absence of spontaneous sparks (3.1.4.2 E). This confirms the proposed role of RyR3 in amplification of the calcium release initiated by the RyR1-DHPR interaction. Perni et al., 2015a.

\subsubsection{Crooked Neck Dwarf Chicken}

The crooked neck mutation in chicken (cn/cn) is embryonic fatal in the homozygot. The defect has been traced to lack of $\alpha$ RyR (RYR1) while $\beta$ RyR (RYR3) is expressed. The latter does not sustain e-c coupling and embryonal development is severely impaired and eventually lethal. At early stages of in ovo development muscle from WT chick shows normal development of CRUs, with condensed CASQ and junctional feet in triads (3.1.4.3 A). cn/cn muscles show rare SR to T tubule junctions, with a dispersed CASQ, occasional feet and some evidence for a periodic junctin-triadin presence (3.1.4.3 B, C). Airey et al., 1993.

\subsubsection{Role of DHPR $\beta$ Subunit}

The paralyzed zebrafish strain relaxed carries a null mutation for the DHPR $\beta 1$ a subunit, resulting in reduced expression of the pore forming DHPR a1S subunit; elimination of $\alpha 1 S$ charge movement, failure of e-c coupling and lack of DHPR tetrads (not shown). Functional and structural defects are fully restored by expression of fish or mouse $\beta 1 \mathrm{a}$. The restoration critically depends on expression of the skeletal muscle $\beta 1$ a subunit that results in the formation of well defined groups of tetrads (3.1.5 A). Substitution of the $\beta 1$ a subunit from muscles of Musca (an insect) is partially 
successful in the restoration (3.1.5 B), but the cardiac/neuronal $\beta 2$ a. subunits is not effective (3.1.5 C). See also 3.1.2 for contributions of Musca subunits, an indication of the ancient origin of DHPRs. Schredelseker et al., 2009; Dayal et al., 2010.

\subsubsection{RyR-DHPR Double Knockout}

Double knockout mice, with null expression of RyR1 and DHPRs, were engineered starting from dysgenic (DHPR null) mice. The muscles were harvested at embryonal day 18-19 embryonic age, since the mutations are lethal at birth. WT myotubes show peripheral foci of $4 \mathrm{CRU}$ components (RyR1, DHPR, triadin and calsequestrin), while double mutants lack the first two but maintain clustering of triadin and calsequestrin (3.1.6 A). The late embryonal muscles are poorly developed relative to the wild type ones, due to lack of contractile activity, but primitive triads are present (3.1.6 B). Comparison of embryonal triads (3.1.6 C) from wild type (A), dysgenic (DHPR null, B), dyspedic (RyR null, C, D) and double KO muscles $(E)$ shows that assembly of triads does not requite either of the two major components, see also 316C. Interestingly, the periodic arrangement of triadin is not affected (compare with 2.5.2.4). Felder et al., 2002; Protasi et al., 2002.

\subsubsection{Stac3}

Stac3 is a recently discovered component of CRUs, responsible for facilitation expression of DHPRs in the junctions and forming part of the direct link between RyR and DHPR. STAC3 experiments are discussed in 2.5.2.3 Stack3.

\subsection{Null Mutations and Overexpression}

\subsubsection{Cardiac CRU Proteins Overexpression}

Calsequestrin (CSAQ), triadin and junctin form part of the supramolecular complex of CRUs. Overexpression of the three proteins in cardiac muscle have striking structural effects (see also 2.5.2.4). Overexpression of CASQ inflates SR cisterns with a gel of the polymerized proteins (3.2.1 A, B), eliminating the difference between the tight, narrow, cardiac jSR and the extended skeletal one (3.2.1 C). Junctin has the opposite effect: its over expression tightens the cardiac jSR making it very narrow (3.2.1 D) and greatly extending jSR cisternae even where they are not associated with CRUs (3.2.1 E, F, G). In conjunction with CASQ junctin reveals its role in linking CASQ to the jSR membrane (3.2.1 H). Jones et al., 1998; Zhang et al., 2001; Tijskens et al., 2003b; Boncompagni et al., 2012a.

\subsubsection{Cardiac Junctophilin Overexpression}

Junctophilins are proteins that mediate docking of ER/SR to the plasmalemma. Over expression of junctophilin-2 (JP2), the cardiac isoform, in cardiac myocytes attenuates cardiac failure after stress. and alters dyad geometry, with an excess of jSR to T tubule docking. WT CRUs are composed of a single jSR element that wraps around $T$ tubules, occasionally appearing as two separate profiles (3.2.2 A, see 2.5.1.2). JP2 over expression results in the association of multiple T tubule profiles with multiple jSR cisternae (3.2.2 B, C). Guo et al., 2014.

\subsubsection{Engineered Mutations}

\subsubsection{Skeletal CASQ Null}

A null mutation for the skeletal muscle specific calsequestrin results in profound effect on the geometry of skeletal muscle CRUs (Paolini et al., 2007). Multiple jSR-jT asssociations results in pentad and heptads in place of the normal triads and the junctional membranes are extended into extended RyR-containing plaques (3.2.3.1 A, B). DHPR adapt to the new 
CRU shapes by forming large arrays of tetrads (3.2.3.1 C, D. S. Boncompagni, unpublished). Paolini et al., 2007.

\subsubsection{Cardiac CASQ Null}

The contribution of cardiac CASQ to the normal ventricular beating and to the structure of cardiac SR in triads is evidenced by the results of null mutations. Deletion of cardiac calsequestrin (Casq2) results in ventricular tachicardia and junctional $\mathrm{SR}$ cisternae that are empty and dilated and lack the periodic arrangement of dense content (3.2.3.2 A and B, see also 3.2.1 for the converse effect of overexpression of junctin). Reduced expression results in slight enlargement of the jSR (3.2.3.2 C). Substitution of a normal with a D307H mutated CASQ does not affect the structure (3.2.3.2 D). Knollmann et al., 2006; Chopra et al., 2007; Kalyanasundaram, et al., 2010.

\subsubsection{SR ATPase Reduction}

In cardiomyocytes, $\mathrm{Ca}^{2+}$ is cycled across both the plasmalemma and the membrane of the sarcoplasmic reticulum (SR). These two systems interact closely during e-c coupling and their relative contributions depend on the heart of origin and on status-dependent regulations. In normal murine heart, SR release contributes about half of the e-c coupling calcium, the rest comes from extracellular sources. At equilibrium the balance is maintained by $\mathrm{SR} \mathrm{Ca}^{2+}$ pump (SERCA) and by a plasmalemma transport ATPase. Interestingly, reduction of SERCA to extremely low levels is not immediately lethal. However, the free SR changes its configuration. WT free SR has a small but well defined luminal volume (3.2.4 A, B). After 4 weeks of SERCA reduction, the free SR volume is greatly reduced and some of the SR is totally collapsed (3.2.4 C, D). After 7 weeks the majority of the SR is collapsed (3.2.4 E,F). jSR in the dyads is spared (3.2.4 G). Contrast enhancement by tannic acid enhanced visualization of SERCA as a dense band on the SR cytoplasmic surface. This technique confirms reduction of SERCA in the treated specimens (3.2.4 H). Swift et al., 2012.

\subsubsection{Cardiac Triadin and Junctin Null Mutations}

Ablation of triadin in engineered mouse cardiac muscle has a strong structural effect, because it also results in decreased CASQ and junctin expression. WT SR combines a network of free SR (3.2.5 A) and numerous jSR-T dyads (3.2.5 B). The main change in triadin null cells is in the reduced extent of SR-T junctions and in the wider lumen of the jSR presumably due to lack of CASQ (3.2.5 C). The positioning of RyRs in dyads (3.2.5 D) and the free SR structure (3.2.5 E) are not affected. Ablation of junctin has the interesting effect of multiplying the jSR-T connections so dyads often have multiple layers (3.2.5 F, G). Chopra et al., 2009; Boncompagni et al., 2012a.

\subsection{Expression in CHO Cells}

Chinese Hamster ovary $(\mathrm{CHO})$ cells do not express muscle specific proteins and thus are an appropriate platform for studying their experimental expression. RyR1 and the five subunit of CaV1.1 (skeletal muscle DHPR) were first successfully co-expressed in cultured $\mathrm{CHO}$ cells in 1995. RyRs were present at high levels in the cytoplasm, where they assembled into elongated arrays of multilayered ER bearing semicrystalline arrays of feet (3.3 A-D). The intense needlelike foci of RyRs are easily detected by immunolabeling (3.3 E). Interestingly, similar RyR assemblies form in the cytoplasm of blowfly larvae during metamorphosis when other SR proteins are absent $(3.3 \mathrm{~F})$. Feet decorate the isolated SR membrane, fractions, but their disposition is disordered (3.3 G). The alpha1 subunit of DHPR is expressed in the plasmalemmas, but it fails to associate with RyRs, so although groups of DHPR particles are present, no tetrads are formed $(3.3 \mathrm{H})$. An additional component, later identified as stac3, is needed for complete CRU formation (Perni et al., 
2017).

Frequency of ER targeting to and association with the plasmalemma is enhanced by expression of junctophilin (33 I) and co-expressed feet occupy the peripheral couplings (3.3. J). Takekura et al., 1994b, 1995a, b; Crossley, 1972; Barone et al., 1998; Perni et al., 2017.

\subsection{Myosin Exchange in Drosophila Muscle}

All muscle myosins in Drosophila are encoded by a single myosin heavy chain gene and expression of muscle-specific isoforms is regulated by exon splicing. The indirect flight muscle (IFM) differs significantly from that of other body muscles both in overall sarcomere structure (thin/thick filament ratio, A band length etc) and in the frequency and disposition of dyads and mitochondria. In order to determine whether myosin isoforms play a role in differentiation of the flight muscle, a transfly was generated in which the myosin for the jump muscle (TDT) was expressed in a myosin-null flight muscle. In WT muscle the thin/thick filament double hexagonal array is flawless even at the myofibril edge (3.4 A, see 2.1.5.3 B). In the trans fly the center of each myofibril achieves the same order as the wild type, but the edges are disordered with variable thin/thick filament ratios (3.4 B, C). Dyad structure and disposition are the same as in WT. The myosin inform does not affect the major structural components of the flight muscle, and the peripheral disorder of the myofibrils is probably indicative of incomplete differentiation.

\section{Part 4 Age and Pathology}

\subsection{Age Related Alterations}

\subsubsection{CASQ and Tubular Aggregates}

In addition to an overall decrease in size of most components (sarcopenia) the fast skeletal muscle fibers of aging mice show an unexpected accumulation of calsequestrin (CASQ) and the formation of tubular aggregates perhaps as a means of segregating the extra CASQ. Initial accumulation of CASQ is in the I band SR, which becomes inflated (4.1.1 A, B). Triads may also become dislocated, taking a longitudinal orientation (4.1.1 C) and the SR is convoluted (4.1.1 D). Small tubular aggregates form in conjunction with dilated SR (4.1.1 E) and new SR and triads add to the edge of the aggregates (4.1.1 F, G, H). The initial short tubular segments are randomly disposed $(4.1 .1 \mathrm{I})$ and they become parallel in the larger assemblies $(4.1 .1 \mathrm{~J}, \mathrm{~K})$. Semicrystalline arrangement of the calcium ATPase in the tubules is probably responsible for restraining the SR into tubular form $(4.1 .1 \mathrm{~L})$. Immunolabeling shows RyR clusters at the edges of the aggregates and CALSQ in the centers (4.1.1. M). Boncompagni et al., 2012.

\subsubsection{Vernier Displacements and Z Line Streaming}

Vernier displacement of the cross striation occurs where the striations of two adjacent myofibrils are mismatched because fibril on one side contains more sarcomere than the opposite side over limited length (4.1.2 A-C), see also 2.1.4.2. Vernier mismatch imposes a stress on the sarcomeres and it is often accompanied by $Z$ line streaming particularly in postural muscles (4.1.2 A-E). Excessive streaming disrupting the sarcomeres over a wide area is usually coupled to pathological conditions (4.1.2 F, G). Pellegrino and Franzini, 1963; Boncompagni et al., 2009, 2010.

\subsubsection{Mitochondria Redistribution}

Type 1 (slow fatigue resistant) fibers in mouse have clusters of mitochondria at the fiber's edge, see 2.7.2.2. This 
distribution is exaggerate in aged muscles, where large mitochondria accumulations are near areas where intermyofibrillar mitochondria are absent (4.1.3 A). It also occurs in pathological conditions see 4.2.2.A..

\subsection{Mouse Models of Malignant Hyperthermia}

Human malignant hyperthermia $(\mathrm{MH})$ is a mutation leading to a potentially lethal elevation in muscle temperature. $\mathrm{MH}$ susceptible patients remain subclinical until challenged with one or more pharmacologic triggering agents, including halogenated volatile anesthetics, depolarizing neuromuscular blockers, and/or heat stress. The defect is hereditary and over the years it has been traced to a number of alterations in genes expressing e-c coupling related proteins, mostly RyR and DHPRs, but also others.

A survey of human muscle biopsies from proven $\mathrm{MH}$ susceptible patients has identified a number of subtle structural defects, but not a specific-disease related alteration. A more direct approach has been to express in mice the well identified mutations obtained from human genetic studies and closely study functional and structural defect. The approach has been quite successful in a number of cases. Lavorato et al., 2016.

Lavorato M et al., 2016.

\subsubsection{RyR1 ${ }^{\text {Y522S }}$ Mutation, Central Core}

The RyR1Y522S mutation is linked to malignant hyperthermia and to central core disease in humans. "Central core" fibers are characterized by a central region that lacks mitochondria and other components. The mouse models of the RyR1Y522S mutation turned out to be most interesting in terms of structural information, because they allowed a follow up of the gradual changes that lead to the formation of cores. In the heterozygote there is SR Ca2+ leak and overproduction of oxidative species of oxygen and nitrogen with subsequent mitochondrial damage. Early changes involve alterations of mitochondria (4.2.1 A, B). Early pre-cores or mini cores indicate local alterations (4.2.1 C, D). Localized or diffuse disorder of the striation is evidenced by immunolabeling of RyRs (4.2.1 E) and larger areas are similarly affected (4.2.1 F). In the postural soleus muscle large and small areas of $Z$ line streaming indicate expansion of the pathology (4.2.1 G). Loss of mitochondria and SR and failure to control calcium handling leads to extensive contractures further damaging extensive areas $(4.2 .1 \mathrm{H}$, I). Boncompagni et al., 2009.

\subsubsection{RyR1 ${ }^{\mathrm{T} 4861}$ Mutation}

The RyR1T486I mutation confers MH susceptibility in heterozygot humans and HM episodes are confirmed in homozygote mice with the isogenetic mutation. Structural alterations in homozygot mice include abnormally distributed mitochondria due to loss of normal tethering links holding the mitochondria in position (4.2.2 A, B). At a finer level triads are also displaced (4.2.2 C). $Z$ line streaming is indicative of stress to the contractile system 4.2.2.D (also seen in aging muscle, see 4.1.2). Lysosomes are more frequent than normal, indicating structural breakdown (4.2.2 E-G). Yuen et al, 2012.

\subsubsection{RyR1 ${ }^{14895 T}$ Mutation}

The RyR114895T mutation results in lack of calcium release from the mutated ryanodine receptor. Not surprisingly the embryonal muscles from the homozygous mutant resemble quite closely those in two other models lacking RyR calcium release: RyR1 null mouse (not shown) and the crooked neck dwarf (see 3.1.4.3 folder).

The heterozygot's function is not strongly affected, but structural changes are indicative of "wear and tear". Accumulation 
of CASQ in I band vesicles (4.2.3 A, B is similar to that seen in aging muscle (see 4.1.1) but more exaggerated. Longitudinal triads $(4.2 .3 \mathrm{C})$ and $\mathrm{Z}$ line streaming (4.2.3 D, E) are result of stress to the myofibril. $T$ tubule swelling is unusual (4.2.3 F). Extensive contractures are present in the more affected muscles (4.2.3 G). Zvaritch, et al., 2007; Boncompagni et al., 2010.

\subsection{Modeling Cardiac Mutations}

\subsubsection{Cardiac CASQ ${ }^{\mathrm{D} 307 \mathrm{H}}$ Mutation}

The structure of cardiac jSR cisternae is classified as "tight" if the cisternae have a small width and CSQ disposed in periodic densities as in wild type myocardium (4.3.1 A). "Loose" jSR cisternae, as in CASQ null myocardium, are apparently empty and the width is larger (see 2.5.2.3.2 B). The CASQD307H mutation is linked to catecholaminergic polymorphic ventricular tachycardia. When expressed in a CSQ2 null background the mutant protein is stable and it partially rescues the CASQ null jR structure by tightening the cisternae (4.3.1 B). It also induced some proliferation of T tubules (4.3.1 C). Kalyanasundaram et al., 2010.

\subsubsection{Mitochondria-Related Mutation}

A RyR2 mutation linked to catecholaminergic polymorphic ventricular tachycardia (A4860G) induces a calcium imbalance by depressing RyR2 channel activity during excitation-contraction coupling. Cardiac myocytes of heterozygous mice carrying the mutation exhibit an unusual structural response: an increase in the frequency of mitochondrial nanotunnels (4.3.2 A) that allow a slow exchange between mitochondria, see 2.8.5 D. Lavorato et al., 2017.

\subsection{Zebrafish Twister}

Neuromuscular transmission is prolonged due to to a gain-of-function of the $\alpha$-subunit of the muscle-specific AChR (CHRNA1) in zebrafish twister mutants A4860G causing motor axon extension. Developing muscle fibers show ultrastructural evidence of muscle degeneration (4.4 A). Myofibril disarrangement is milder in heterozygots, but very strong in both fast and slow homozygot fibers (4.4 B). Lefebvre et al., 2004.

\section{Part 5 Techniques}

\subsection{Standard Techniques for Thin Sections}

The steps in the most commonly employed approach to preparing tissue for thin section electron microscopy involve: 5.1.1 exposure/dissection of tissue; 5.1.2 chemical fixation; 5.1.3 contrasts enhancement, "en-block staining"; 5.1.4 dehydration; 5.1.5 embedding; 5.1.5 sectioning and 5.1.6 section staining. Below is one of several possible fixation-embedding procedures, with alternative variations. Room temperature is used throughout. See also notes for specific procedures. FIXATION:

3-6\% glutaraldehyde in $0.1 \mathrm{M}$ cacodylate buffer $\mathrm{pH} 7.4$ at room temperature for at least 20 minutes before further dissection. Continue fixation for at least one hr.

Up to $9 \%$ glutaraldehyde can be used. Fixed tissue can be stored at $4^{\circ} \mathrm{C}$ for several month provided the fixative is renewed at monthly intervals. 
Wash in cacodylate buffer 3 times for 10'

Tissue can be stored overnight in the buffer wash solution

POSTFIXATION:

$2 \%$ OsO4 in $0.1 \mathrm{M}$ cacodylate buffer $1 \mathrm{hr}$ at RT

Alternative: post-fixation in $2 \% \mathrm{OsO} 4$ and $8 \% \mathrm{~K} 3 \mathrm{Fe}(\mathrm{CN}) 6$ in $0.1 \mathrm{M}$ cacodylate buffer

Wash in $0.1 \mathrm{M}$ acetate buffer $\mathrm{pH} 4.53$ times for 10'

En-block STAINING with uranyl acetate. Usually not done if the ferricyanide is used

Use saturated uranyl acetate solution in acetate buffer, better prepared the day before

\section{DEHYDRATION:}

The suggested times are for small tissue samples.

Rinse in $70 \% \mathrm{EtOH} 1$ time for 10 min at RT

If needed, tissue can her stored overnight at $4^{\circ} \mathrm{C}$

Rinse in $95 \% \mathrm{EtOH} 1$ time for 10 min at RT

Rinse in pure EtOH 3 times for 10 min at RT

Rinse in acetone 3 times for $10 \mathrm{~min}$ at RT

\section{INFILTRATION:}

Infiltrate with a mix of acetone and resin 1:1 (we use epon) - $1 \mathrm{hr}$ at RT

Move tissue to a small pool of pure resin for a few minutes, then put in embedding mold and leave it for at least $1 \mathrm{hr}$ to be sure that all acetone is evaporated.

Alternatively: Keep tissues that are difficult to infiltrate and tight cell pellets under moderate vacuum overnight to eliminate all traces of acetone.

\section{EMBEDDING:}

Polymerize in resin, over night at $60^{\circ} \mathrm{C}$ (for epon).

\subsubsection{Preparing Tissue}

\subsubsection{Dissection}

Chemical fixatives do not penetrate well so the tissue of interest must be exposed. One possibility is to mince the tissue into small cubes. This works well for tissues composed of small cells, like liver. However, muscle fibers are long and they must not be damaged before fixation to avoid contractures etc. Slicing and/or cutting the tissue before fixation is simply not done Muscle must be carefully dissected, including tension extensions. Once dissected the muscle is pinned in a dish to avoid distortions and flooded with fixative.

If a good dissection is not feasible, for example in the case of fish myotomes or for the diaphragm in small mammalian embryos, the muscle should be exposed and bathed in fixative. After a short period the superficial layer is "fixed" and can then be removed by cutting. In the extreme case of very small organisms fixation is through the skin, but it requires a higher concentration of fixative. Muscle exposure in arthropods require removal of the chitin later. This can be achieved by gently scraping with a razor blade.

In the cause of isolated cells either in culture or in suspension, care must be taken to wash out culture media using a 
balanced saline before exposing the cells to the fixative, because the media proteins neutralize some of the fixative activity. Cells in suspension may be fixed either by dispersing in the fixative or by pelleting first and then fixing the pellet as if it was a piece of tissue. Note that in the latter case penetration of the fixative must the taken into consideration: the size of the pellet should be small.

\subsubsection{Fixation in situ}

In cases where dissection is particularly difficult, for example in the long thin legs of "daddy long legs" the difficulty is solved by simply injecting the fixative inside the leg.

\subsubsection{Fixation by Perfusion}

Fixation by perfusion is possible in vascularized organism and it is the best approach for organs that may be difficult to access and /or to dissect. Brain and the thick layers of myocardium are two obvious examples. The principle is simple: an appropriate blood vessel is cannulated and the fixative is injected into it. Upon reaching the organ's capillaries the fixative acts rapidly through the entire volume. An initial perfusion with a saline high in potassium is often used in order to wash out blood serum/blood cells and to prevent contractions in blood vessels' smooth muscle. Retrograde perfusion through the left ventricle is often successful for fixation of ventricles, but direct access to the coronary circulation via the aorta, as in the in preparation for the Langendorff isolated heart perfusion technique (see Wikipedia), is the best approach.

\subsubsection{Biopsies}

The necessity for biopsies is encountered either when the intention is to fix part of the muscle and use the rest for other purposes, or in the case of human muscles where of course extensive dissection cannot be used. The essential biopsy technique first developed by Andrew Engels is applicable in both cases. The principle is that bundles of muscle fibers on the exposed surface of the muscle are gently teased apart, but not dissected. Then the two ends of the bundle, at a distance of $\sim 2 \mathrm{~cm}$ from each other are either tied to a small wood stick lying at their side, or clamped and held in place by to special clamps devised by Andrew G Engel. Finally the bundles are cut away and immersed in fixative. Note: the muscle bundles are now cut, but their fibers are not allowed to shorten, and the damaging effect of calcium entry through the cutting does not have time to spread by diffusion to the center of the bundle. Most of the biopsy length is very well preserved.

A second approach is best for patients (human or other). A relatively large, sharp needle is plunged onto the muscle and on retraction a device allows recovery of the small muscle sample within the needle lumen. This approach, with its rapidity and limited tissue damage, is perfect for biochemical and molecular assays, but it is severely limited by structural considerations. One problem is the random orientation of fibers in the recovered sample. A second problem is that damage has a chance to affect the entire very shot fiber segments before fixative can stop it. We sought to ameliorate these problems with limited success, using two approaches. First the biopsy is extruded into a high potassium solutions strongly chelated for calcium. The fibers are thus depolarized and relaxed, while extracellular calcium does not diffuse into the fibers. Secondly, small bundles with a coherent fiber orientation are teased apart. Pietrangelo et al., 2013; Lavorato et al., 2016; Engek and Franzin-Armstrong, 2004

\subsubsection{Primary Chemical Fixation}

Chemical fixation is achieved by exposing the tissues/cells to appropriate solutions that block activity of the cell 
components and stabilize the structures, e.g., by protein cross linking. Primary fixatives are solutions of formaldehyde at a low concentration (2-4\%) and glutaraldehyde at concentrations of $2-9 \%$. The two may be applied sequentially or mixed, formaldehyde is not always essential. Formaldehyde penetrates fast and provides a first, if weak, structural stabilization; glutaraldehyde is a cross linker of protein and thus it provides a strong stabilization. All solutions are buffered to near neutral $\mathrm{pH}(7.2-7.4)$ by a fairly concentrated $(0.1 \mathrm{M})$ buffer. Buffers based on either phosphate or cacodylate are appropriate for the $\mathrm{pH}$ range needed. The latter has the advantage of not reacting with the calcium of the extracellular matrix.

All fixatives are available from Electron Microscopy Sciences. 1560 Industry Rd, Hatfield, PA. Formaldehyde comes either in a fairly stable solution (different from the "formalin" used for histological or cadaver work) or as a purified polymerized paraformaldehyde powder that may be kept for a long time and used to prepare buffered formaldehyde solution with short life span. Glutaraldehyde slowly polymerizes when in a diluted solution. It should be stored as a $50 \%$ stock and diluted within few hours of use. $6-9 \%$ concentrations are needed when the fixative has to penetrate trough skin (e.g., in a zebrafish larva) or thick fascia. In most cases of dissected muscle a 3-4\% concentration is sufficient. NOTE: glutaraldehyde fixation is slow (minutes) and thus it cannot be used to stop events in action.

\subsubsection{Contrast Enhancement}

Enhancement of image contrast for thin section electron microscopy (inappropriately called "staining") is based on the addition to the fixed structures of high atomic number elements that increase scattering of the electron beam. See Lavorato and Franzini-Armstrong, 2017

The first steps are done in the embedding procedure. Standard postfixation in $\mathrm{OsO} 4$ stabilized lipids and adds a limited, but often sufficient, contrast. Osmium tetraoxide $(\mathrm{OsO} 4)$ is available either in a $4 \%$ aqueous solution or as a crystal in sealed glass ampoules. These can be used to obtain the stock $4 \%$ solution that, unless contaminated, is quite stable. Mixing the stock $4 \%$ with equal volumes of $0.2 \mathrm{M}$ neutral $\mathrm{pH}$ buffer yields the $2 \%$ OsO4, $0.1 \mathrm{M}$ buffer commonly used for post fixation. NOTE: OsO4 is extremely volatile and very dangerous. It must be handled under a hood, and kept in well sealed containers.

3-4 washes in buffer are interposed between the different solutions throughout the embedding procedures.

\section{Further contrast enhancement techniques.}

1. "en bloc" procedures. Exposure of the tissue to an aqueous solution of uranyl acetate between $2 \%$ and saturated for one half to one hr greatly enhances the contrast of structural proteins (e.g,, muscle filaments). NOTE: uranyl acetate is not soluble at neutral $\mathrm{pH}$. To avoid microprecipitation of dense uranium dots in the tissue it is essential to interpose either multiple $\mathrm{H} 2 \mathrm{O}$ washes or 2-3 brief washes in $0.1 \mathrm{M}$ acetate buffer $\mathrm{pH} 4.2$ between the buffered $\mathrm{OsO} 4$ and the uranyl acetate solutions. An alternative approach is to mix $\mathrm{K}_{3} \mathrm{Fe}(\mathrm{CN})_{6}$ with the osmium postfixative. This introduces a high contrast of membranes, thus enhancing visibility of mitochondria, ER/SR etc, see figure, but adding little contrast to protein components (e.g. sarcomeres, microtubules etc.). Potassium ferrocyanide and uranyl acetate have two opposite effects on glycogen granules: they are enhanced in the former, but basically invisible in the latter, see 2.7.2.1 G: 2.7.2.1.B.

2. Mordating by low molecular weight tannic acid (TA) binds reduced OsO4 to various structures. A striking effect in muscle is to reveal the cytoplasmic extensions of the calcium. ATPase in the free-SR surface (see 2.3.2 B and 3.2.4 
H). TA can be added to the primary fixative, or between primary and secondary fixations, however it does not penetrate very well and it results in precipitates if extensive washings are not used between it and the OsO4 and Uranyl acetate solutions.

\subsubsection{Dehydration}

Dehydration is a series of steps by which water in the fixed and stained sample is substituted by polar solvents: ethyl alcohol and acetone. A standard approach is to immerse the tissue in graded series of increasing solvent concentration, Balancing of the osmolarity is not needed because the fixed tissue is not osmotically sensitive. A standard series is $70 \%$, 95\%, 3 times absolute EtOH, 3 times acetone. For very small samples 1 minute for each step is sufficient, for large samples 15-20 minutes are better. Care must be taken that the final 100\% EtOh steps involves very dry absolute EtOH. For the other steps the solutions can be obtained by diluting the less expensive $95 \% \mathrm{EtOH}$. $\mathrm{MeOH}$ may be substituted for $\mathrm{EtOH}$. The final steps in purified acetone are required for allowing infiltration of the embedding resin.

\subsubsection{Embedding}

\subsubsection{Organs and Tissues}

For thin sectioning the tissue is embedded in an appropriate plastic. This requires substitution of non polymerized resin for the final dehydration solvent followed by elimination of the solvent and polymerization of the resin. Acetone is a good solvent for epoxy resins that are standardly used in the US and for Araldite, a resin preferred in the UK, that allows "staining"'of the sections in permanganate.

The tissue is infiltrated with the resin diluted 1:1 in acetone, followed by the undiluted resin before placing in one of the commercially available molds. 1-2 hrs at room temperature are sufficient for evaporation of the remaining acetone, but if needed an overnight exposure to a mild vacuum is also recommended. Polymerization is in a small oven at 60-650 F. Overnight polymerization is sufficient, but best sectioning is obtained after a few days. Older blocks, months or years after polymerization, offer the best sectioning, presumably because the resin is more completely cross linked to itself. See also a description of the entire protocol (5.1).

\subsubsection{Isolated Cells and Organelles}

Isolated cells present additional challenges. First, dehydration and embedding is carried out with the cells in the same small tubes (e.g.,1.5 ml Eppendorff) so the cells are not dispersed and lost. If the cells are initially fixed in suspension they tend to float and thus they need to be pelleted by mild centrifugation at each solution change. If the cells are fixed as a pellet, then it important that the pellet in not too large, because the fixative may not permeate through the entire pellet. The final steps: infiltration in 1:1 and in undiluted resins and polymerization are performed without taking the cells out of the tube. HOWEVER: this may result in some residue of acetone in the final resin. If this is the case polymerization is defective and it cannot be fixed by a long cure in the oven. The final pellet must be treated in a low vacuum chamber at least overnight to allow complete evaporation of acetone residues.

Isolated muscle cells (such as derived from rat/mouse FDB; fish myotomes; myocardium) are elongated and they would be poorly oriented in a pellet. A final step is essential: a small drop of the final pellet in resin is placed over a small sample of aclar plastic available from EMS. Acetone evaporates and the cells lie flat on the plastic surface. After polymerization 
the thin sheet of resin is removed from aclar and glued to a prepolymerized cylinder of resin, ready to be sectioned (see 1487A, M; 1485 A; 1486G). Huang et al., 2013.

\subsubsection{Thin Sectioning}

\subsubsection{Sectioning and Staining}

Most "standard" Transmission Electron Microscopy of cells and tissues involves a view by transparency of sections that are very thin $(\sim 50-90 \mathrm{~nm})$ in order to reduce the number of interactions of the electron beam with the heavy atoms in the tissue that result in loss of the beam coherence and thus in decay of resolution due to chromatic aberrations.

Embedded tissue is sectioned using special ultramicrotomes using diamond knifes. The embedded bloc is trimmed to a small pyramid either with a glass knife or with an old obsolete diamond knife. The water level in the knife boat must be carefully arranged to provide a clean reflection so that section thickness (see below) and quality can be judged. A diamond knife lasts forever provided that care is taken to avoid chipping it. Keep water clean: use a syringe to take water off the diamond boat and a small water bottle to add water.

A special compressed styrofoam stick (EM Science) is used to clean the diamond edge, but the best is to operate so that such cleaning is seldom needed.

After sectioning, chloroform evaporating from a wood stick moved in proximity of the sections flattens them. Be most careful to avoid contact of the stick with the boat water.

Section thickness is determined by setting in the ultramicrotome. However, various thermal effects may modify the final thickness. The best is to get a rule of thumb for judging the section based on the color due to interference of beams reflected from the bottom and top of the section, when water level and illumination are optimized. If the embedding medium is Epon, silver to gold sections are fine for low resolution; silver sections are good for most imaging, but not for the highest resolution; dark pewter to almost black sections are very thin and used for special purposes.

\subsubsection{Collecting sections}

For insertion into the microscope the sections are supported by a fine mesh copper grid that fits in the microscope specimen holder. The section is visible between the grid bars.

Commercially available grids come with very thin bars and a variety of mesh sizes, usually indicated by the number of bars per unit distance.

Several choices are available. The simplest is to collect the sections on a "naked" grid. This works very well for sections of well polymerized Epon that are not too thin. A "400 mesh" grid works very well for medium thickness sections. Smaller mesh size grids, that offer wider fields of view should be used for thicker sections and higher mesh grids are better for thinner sections. The sections are picked up from the boat water by lowering a grid under the section and then lifting it with the sections attached.

Three problems are encountered with naked grids due to the fact that the grids, even when kept stored in tight containers, acquire a thin layer of oxidized copper that makes them hydrophobic. The first is that the grid is hydrophobic and sections tend to move away from the grid as it is lifted. The second is that the water accompanying the grids retracts into a rounded bubble and the sections get wrinkled when the bubble dries. Finally, the sections do not adhere well to the grid and they come off when they go through the staining procedure. 
Problem solutions: 1) keep the grids in the tight original containers that are opened as little as possible, thus limiting oxidation. 2) "Clean" the older grids by a very brief (minutes) exposure to $10 \%$ acetic acid followed by a through rinse in $\mathrm{H} 2 \mathrm{O}$ and $\mathrm{EtOH} .3)$ After drying the sections, put the grids in the incubating oven ( $60-65^{\circ}$ centigrade) for $\sim 10$ minutes, allowing the sections to soften and adhere to the grids more effectively.

Alternatively, sections may be collected on membrane coated grids. Grids coated by a collodion or formvar membrane effectively hold the sections between the grid bars and increase their adherence to the grid. This is a necessary technique when either the sections are very thin or a wide grid mesh is selected so the spaces between the grid bars are larger than the section. Membranes introduce problems: one is the fact that even the thinnest membrane reduces the section contrast; secondly coating grids with membranes is very time consuming; finally the probability of adding contamination increases.

\subsubsection{Section "Staining"}

Even after a good en-bloc "staining" the sections do not have sufficient contrast for excellent images, particularly when very thin. A standard procedure is to expose the sections on grids first to a solution of uranyl acetate and second to a lead solution. If the en-bloc staining was successful the uranyl acetate is not essential for medium thickness sections if but it is necessary for better detailing of thin sections

Uranyl acetate penetrates into the sections better when mixed with some alcohol (e.g., 50\%) than in simple $\mathrm{H} 2 \mathrm{O}$ solution, but the alchohol based solution is not stable. The problem is solved by maintaining a stable saturated solution of uranyl acetate in $\mathrm{H} 2 \mathrm{O}$ in the dark. Just before use, mix a small aliquot of the aqueous uranyl acetate with an equal volume of $\mathrm{EtOH}$ in a $1 \mathrm{ml}$ syringe and use immediately. Grids are completely immersed in small drops of the solution on a plastic sheet for 5-10'. Wash in 50\% EtOH by repeated drops from a drop bottle.

Recipes for lead "stains" are quite varied, and most are not very stable. The "Sato" solution (published in 1983) is hard to make, but lasts for several months if aliquoted into $10 \mathrm{ml}$ syringes to avoid contact with $\mathrm{CO} 2$ and gives excellent contrast. Discard when a visible precipitate appears in the syringe.

Preboil distilled water to get rid of $\mathrm{CO} 2$. Calcinate $0.2 \mathrm{gr}$ of lead citrate by heating on a hot plate for $5 \mathrm{t}-10$ minutes until it turns light brown. Add it to a $50 \mathrm{ml}$ flask with $41 \mathrm{ml} \mathrm{H} 2 \mathrm{O}, 0.15 \mathrm{gr}$ lead acetate; $0.15 \mathrm{gr}$ lead nitrate; $1 \mathrm{gr}$ sodium citrate. Shale vigorously until solution is milky. Clear the solution by adding $\sim 9 \mathrm{ml}$ of $4 \% \mathrm{NaOH}$ freshly diluted from a $40 \%$ stoc dropwise. Filter out coarse precipitate. Store in syringes. Use a Millipore filter to get droplets of the stain on plastic in a covered petri dish containing a small amount of $\mathrm{NaOH}$ pellets. Stain for 5-7 minutes, wash in $\mathrm{H} 2 \mathrm{O}$, as above.

Permangante also gives excellent contrast enhancement, However it only works on material embedded in English Araldite, Wu et al., 2010.

\subsection{Additional Techniques}

\subsubsection{T Tubule Labeling}

The lumen of $\mathrm{T}$ tubules is a privileged compartment because it is accessible from the extracellular space and its narrow dimensions slow down loss of luminal content by diffusion Several approaches have taken advantage of these properties in order to specifically label T tubules and allow their identification in thin sections.

A direct approach is to soak the muscle in a balanced salt solution containing a high concentration of ferritin, a molecule 
that is detected as a small dot in the images. See 1.4.8.5 K. Ferritin provided the first EM demonstration that the T tubule is open.

Two infiltration methods use lanthanum ions and horse radish peroxidase as tracers. Gradual change in pH induces the formation of colloidal lanthanum in a calcium-lanthanum solution. After it penetrates into the $T$ tubule the lanthanum precipitates during embedding, resulting is an electron dense precipitate. Horseradish peroxide diffuses in the T tube and becomes detectable after a histochemical reaction.

A more frequently applied tracer results from the addition of $\mathrm{K} 3 \mathrm{Fe}(\mathrm{CN}) 6$ to the osmium post fixation solution. In presence of calcium this results in a somewhat capricious labeling of T tubules and often also of the SR, see 2.13.1 E, 2.11.3 D chick, 2.11.4 A mouse.

Finally the "Golgi" infiltration method results in an extremely dense silver-osmium precipitate in both T tubules and SR. As other methods the results are positive only in a small part of the tissue, but the contrast in the positive area is very high and thus allows the use of semi thin sections at the higher accelerating voltages. e.g., see $2,4.1 ; 2.4 .2 ; 2.4 .4 ; 1.3 .2 .1$ C; 1 .3.2.2 D,E; 1.3.2.3 D, E., 2.7.2.2.I; 2.1.4.2 F.

\subsubsection{Skinning}

"Skinning" provides access of solutes to the cell or muscle fiber interior without altering structural and functional parameters. Mechanical skinning was developed for muscle fibers by the Japanese R. Natori, in 1954. It is obtained by literally stripping off a superficial layer of the fiber using a sharp needle, while leaving the rest of the fiber behind. The technique has been extensively used in functional experiments probing the properties of T tubules and SR. Structurally it has allowed access of oxalate to the SR (see 2.3.2 C) and the probe of internal spaces by ferritin and other fairly large molecules (2.1.3.1.E). A secondary "chemical" skinning procedure that could be used in small cells as well as in muscle fibers involves permeabilizing the surface membrane by various means. Costanti n et al., 1965; Franzini-Armstrong, 1970.

\subsubsection{Fluorescent Membrane Dyes}

Fluorescent lipid probes can be used to label the membranes of T tubules. DilC16 has proven particularly useful because it diffuses readily on the lipid bilayer. The dye stock is maintained in ETOH. Dilution of a small aliquot into Ringer results is a suspension of small micelles. The probability of a contact between the muscle fiber surface and the dye micelles is increased by forcibly streaming the solution over the surface of freshly teased bundles of muscle fibers. A very brief prefixation in diluted glutaraldehyde also works. The usefulness of the sample is greatly limited in time because the dye diffuses away from T tubules. Excellent views of developing T tubules can be obtained. See 2.11.2 A and B. 2.11.3 A.

\subsection{Imaging Small Objects}

\subsubsection{Negative Staining}

The term "negative staining" applies to a technique that surrounds the object of study with an electron dense cover that penetrates into all surface details, but does not penetrate into the object. Thus the latter appears white (transparent) against a dark background. . The most commonly used contrasting agent is a diluted $(\sim 1 \%)$ solution of uranyl acetate in $\mathrm{H} 2 \mathrm{O}$. The technique is simply to apply a small droplet of the sample suspension to a membrane-covered grid (see.....) and to wash off the excess (with an ammonium acetate solution) after the sample adheres to the membrane. Without letting the sample dry, it is then flooded with several successive droplets of uranyl acetate, and the final one is blotted away leaving 
only a very thin layer that is allowed to dry. The solution has the property of drying into a glassy material that surrounds and partly covers the sample (see $2121 \mathrm{~A} ; 2122 \mathrm{~A}, \mathrm{E}, \mathrm{F} ; 2.1$.2.2. E).

The technique is simple, but good results are hard to obtain. The major cause of trouble is the interaction of the negative staining solution with the holding membrane: if not perfect the layer of stain retracts leaving either a blotchy or even a totally failed "staining". Carbon membranes are best in term of "transparency", but they are quite hydrophobic if not recently made. Two solutions to this problem are either to spread a thin layer of a small protein on the membrane prior to use, making it hydrohilic, or to glow discharge the membrane-coated grids under mild vacuum.

\subsubsection{Rotary Shadowing}

Rotary shadowing with heavy metals decorates the surface of small objects revealing structural details. The resolution is lower than in negative staying but the contrast is much higher (see 2.1.2.1 B; 2.1.2.2D; 2.5.2.1 M). "Shadowing" is obtained by evaporating heavy metals (most commonly platinum) under high vacuum conditions while rotating the sample. Angle of shadowing depends on the depth and size of exposed structures. Figs. 2 and 4" in Bard et al., 1987 illustrates the difference between 250 and $45 \mathrm{O}$ shadowing angles in visualizing myosin cross bridges that are recessed between myosin filaments. Shadowing for very small molecules is done at angles of 7 to 15 degrees.

To prepare for shadowing the molecules must be adhered to a clean surface, for example freshly split mica, and dried without structural collapsed due to surface tension of water. This is obtained by two different approaches. One is to freeze the specimens in a very thin laser of solution $(40 \% \mathrm{MeOH}$ works well), then freeze the specimen and dry it under vacuum by sublimation of the solvent. The technique works well with isolated molecules as well a s mall structural elements See 2.1.2.2.D; 2.3.3 D; 2.5.2.1 J; 2.5.2.1 M

A second approach is to use the glycerol spray technique in which a small aliquot of the sample is suspended in a $40 \%$ glycerol solution and then sprayed as small droplets on a freshly split mica using a pressure-driven small nozzle. The glycerol solution retracts into the center of the droplet while a halo of the samples adhering to the mica are protected from surface tension effects in drying. See. 2.1.1 C;2.1.1 D

\subsection{Cryotechniques}

\subsubsection{Freeze-Fracture and Deep Etch}

Freezing may be used as a primary preservation technique, requiring rapid freezing, see 5.4.2, or as a secondary preservation, following glutaraldehyde fixation. When cry-protected in glycerol of methanol, the fixed tissue can be frozen by simply dipping into liquid nitrogen cooled propane for use in the techniques of freeze-fracture and deep etching. If deep etching is required, see below, a volatile solvent is used. 40-50\% MeOh works very well.

For freeze-fracture the specimen is frozen on a small holder which is fitted on a cold holder and mounted on the cold stage of a vacuum evaporator maintained at $\sim-110 \mathrm{C}$. After a good vacuum is obtained the specimen is fractured and the exposed fractured surface are shadowed by platinum and replicated in a thin film of carbon. The replica is cleaned by floating the tissue in sodium hypochlorite solution. The commercially available "clorox" works well. Once the tissue is completely dissolved the replica is washed and mounted on empty EM grids. Two techniques are available for fracturing. One is to use the rotating knife mounted within the vacuum chamber to either crack the specimen in the middle or to shave thin sections using microtome advance until the require area is reached. A second approach, necessary in the case of 
small specimens, is to squeeze the specimen between two thin copper holders, freeze the whole assembly and then separate the two holders under vacuum using an appropriate tool resulting in two complementary double replicas. "Deep etch"is a term commonly applied to the sublimation at low temperature of frozen fluids either from the surface of small objects or from the exposed surfaces of freeze-fractured specimens. Etching is done under controlled temperature conditions, with the tissue maintained at sufficiently low temperatures to avoid water recrystallization during the period of etching, and under vacuum to accelerate the sublimation and avoid contamination of the exposed surfaces. Under high vacuum conditions a few minutes are sufficient to expose details.

Single small molecules frozen on the surface of glass or mica can also be "etched" but in that case the sublimation results in the total extraction of water molecules or in freeze drying.

Image contrast is obtained by "shadowing" with heavy metals (most commonly platinum) under high vacuum conditions while rotating. Angle of shadowing depends on the depth and size of exposed structures .Figs. 2 and 4" in Bard et al., 1987 illustrates the difference between $25^{\circ}$ and $45^{\circ}$ shadowing angles in visualizing myosin cross bridges that are recessed between myosin filaments. See 2.2.4 F; 2.1.4.1 A; 2.4.4.G; 2.5.2.3.1 D; 2.3.3 C.

\subsubsection{Rapid Freezing}

\subsubsection{Catching Rapid Events}

Unfixed tissue can be prepared for electron microscopy under physiological conditions by "rapid freezing": a technique that allows sufficiently fast removal of heat from a specimen so that a peripheral strip $(\sim 5-10 \mu \mathrm{m})$ freezes without disruption of the structure by ice crystals and rapid events are immobilized. The classic way of extracting heat rapidly is to provide a rapid contact between the muscle fiber surface and the highly polished surface of a copper bloc maintained at liquid helium temperature. By this means rapid events such as cross bridge action can be frozen in time. The frozen tissue is either freeze fractured or preserved by freeze substitution in which acetone replaces the frozen water without melting it. See 2.1.2.3 D; 2.1.2.4 D, 2.1.2.4 F; 2.1.2.4 A.

\subsubsection{Pressure Freezing for EM Tomography}

Freezing under pressure allows good preservation in a fairly large specimen. Like other freezings it can be applied to fresh tissue as well as to tissue previously fixed in a low concentration of glutaraldehyde, The latter approach allows dissection of the small samples that are accepted by the pressure freezing machine. The frozen tissue is further preserved by freeze substitution in which the frozen water in the sample is substituted by acetone at a temperature below freezing for water but not for acetone. Exposure to osmium and/uranyl acetate solutions introduce contrast for the images. Freeze substituted samples of pressure frozen tissue are appropriate for 3-D image reconstructions by tomograms of semi thin sections see

2.13.2 C. Lavorato et al., 2017.

\subsection{SEM Techniques}

\subsubsection{Exposing Cell Surfaces}

The scanning electron microscope (SEM) images the free surface of solid objects by detecting secondary electrons omitted by the interaction of a small focused beam with the object surface under vacuum conditions. In preparation for microscopy the object must be dehydrated, coated with a thin conduction metal layer and freely exposed.

Muscle fibers and blood vessels are immersed within connective tissue, so their surfaces are not freely exposed even after 
mechanical dissection. A very effective technique for cleaning muscle fibers and blood vessels of collagen is the OsO4 maceration technique, introduced in Japan. The approach relies on the fact that unbuffered OsO4 slowly solubilizes all structural proteins of the connective tissue (see 2.14.2 B and L) even if partially cross linked by low concentrations of glutaraldehyde. By digesting the basal lamina and the connective tissue of the endomysium, OsO4 maceration of small fiber bundles exposes details of pre- and post-synaptic membranes at neuromuscular junctions; satellite cells nested on muscle fiber surfaces; and pericytes surrounding capillaries. However, the macerating solution does not cross membranes and leaves the surface of all cells intact. Following maceration all membranes are stabilized and also infiltrated with more osmium, which renders them more conductive and so more appropriate for imaging in the scanning electron microscope (SEM). See. 2.14.1 E; 2.14.1.J, K; 2.14.2 E.

The low pH of unbuffered $\mathrm{OsO} 4$ solution is most likely the effective agent, since spectacular view of "cleaned" pre- and post junctional architecture of the neuromuscular junction were obtained by treatment in a high concentration of $\mathrm{HCl}$ (Desaki and Uehara, 1981).

\subsubsection{Exposing Internal Structures}

Maceration extracts only proteins that are on the outside to the plasmalemma, so the cell's and fiber's interior is not revealed. The tissue preparation for exposing internal structures requires a step allowing access the interior of the muscle fiber. A novel method was developed by Tanaka and Mitsushima (1984) and beautifully illustrated by Ogata and Yamasaki in 1990. The initial step is fixation in low concentration of formaldehyde-glutaraldehyde. The tissue is then cryoprotected in $30 \%$ glycerol, frozen in liquid nitrogen-cooled propane and cryofractured at atmospheric pressure. This can be done using the knife on the cold stage of a cryo fracturing machine or more roughly and less reliably by tapping the frozen tissue with a cold razor blade or gently crushing it with a small hammer kept at freezing temperatures. After thawing the tissue is post fixed in $1-2 \%$ buffered OsO4 and then exposed to "maceration" in a low concentration $(0.1 \%)$ of buffered OsO4. Over a prolonged period (up to 90hrs), the osmium solution dissolves all proteins (myofibrils, microtubules etc) leaving behind (and actually preserving) all membranes (SR, T tubules, mitochondria). The tissue is then prepared for scanning EM imaging by infiltration in tannic acid-osmium, critical point dried and coated with metal. Unfortunately, appropriately fractured surfaces are rare and this requires a lot of time at the microscope. However, the results are stunning, revealing mitochondria, SR and T tubules in 3D images see 1.4.8.6 E; 2.8.3 L

\subsection{Immunolabeling}

The technique of immunolabeling at the light and electron microscope levels is based on the availability of primary antibodies with a high affinity for specific proteins (or peptides) and on methods that allow sufficient structural preservation without blocking diffusion of antibodies to the targets. If the primary antibody is conjugated to a fluorescent dye (also called fluorophore or fluorochrome) the position of the antigen can be directly identified by fluorescence microscopy. Since most of the commercially available antibodies are not fluorescent, an indirect technique is commonly used. This involves tagging of the antigen with a primary antibody followed by a fluorophore-conjugated secondary antibody that recognizes the species of origin of the primary one. If primary antibodies from different sources are available this permits double (or even triple) labeling for different proteins. Extensive washes are used between exposures. In the case of tissues, labeling is often done on frozen sections. We developed an alternative approach particularly 
appropriate for muscle. Muscle bundles were fixed in a mild formaldyde solution containing a low concentration of TritonX, then gently teased into small bundles of 1-5 fibers and exposed to the primary antibody overnight. Secondary anybody was used for a briefer period.

The fibers are mounted in an anti-bleach medium and observed within short period to time. See 4.1.1 M; 1.4.8.3 D; 2.5.2.4 Q; 1.4.9.I.

Immunolabeled images are shown in false colors, usually at the emission wave length of the secondary antibody used., but it may also hasher false cool indication intensity of stain: 2.5.2.2.J

\subsection{Histochemistry of Motor End Plates}

Skeletal muscle fibers end plates, although fairly large, occupy a very small portion of a fiber surface. Hence random sectioning very seldom shows end plates. A technique based on a a histochemical reaction identifies non-specific esterases. The substrate is Indoxyl Acetate; the reaction product is coupled to Hexazotized pararosanilin (pararosanilin in Sodium nitrate), which precipitates at the activity sites in the tissue. Once the endplate's location is outlined, it can be observed in the light microscope or embedded for EM. See 2.14.5 G for LM and 2.14.5 H. I for TEM.

\section{Protocol:}

- Muscle fixation in Glutaraldehyde 3\% in Pho buffer for one hour. Muscles fixed for longer times still give positive reaction for the staining up to $\sim 1$ month.

- Wash very well in Pho buffer $5 \times 10$ min

- Incubate with staining solution:

- $0.1 \mathrm{M}$ citrate buffer $\mathrm{pH} 62.5 \mathrm{ml}$

- Hexazotized pararosanilin: $0.15 \mathrm{ml}$ Prepare a mix 50\% each of Pararosanilin and Sodium nitrate $4 \%$.

- Indoxyl acetate $10 \mathrm{mM}: 0.75 \mathrm{ml}$ (prepared in acetone)

- Incubate up to $45 \mathrm{~min}$.

- Wash in buffer Pho $5 \times 10$ min

- The end plates are well visible at the light microscope by teasing small bundles of fibers

Positive regions are selected for EM and cut in small pieces $(1 \mathrm{~mm})$ under the microscope then treated in OsO4, dehydration and embedding, as usual.

* Once embedded, in the semithin sections you should be able to see the positive regions as dark red in the light microscope red regions

* The staining solution must be prepared fresh and it is important to control the solution during staining. If gets "dense" and purple it means that the Hex-Par is precipitating therefore has to be changed for a fresh one. Indoxyl acetate has to be prepared fresh each time also.

* The hex-Par is mixed separately before adding to the staining solution.

* Once in the staining solution the muscle gets red (Brick color) and the reaction sites look as dark spots the reaction should be stopped. Is recommendable to control the staining under the microscope. End Plates Staining Strum and Hall- Craggs, 1982; Csillik and Knyihar 1968, J C science; S Prasarnpun 2004, Neuropharmacology. Mouse NMJ, Matias.Escobar, Kristen Colavita, unpublished images 


\section{Abbreviations and Definitions}

Cardiac: in the context of this presentation, "cardiac" is an abbreviation for "vertebrate cardiac muscle".

RyRs (ryanodine receptors) is the biochemical name for the ERSR calcium release channels. Feet is the name even to RyRs in thin section electron microscopy.

$\mathrm{CRU}$ or calcium release unit is the generic name for all sites of functional connections between $\mathrm{SR}$ and plasmalemma/T tubules that function in e-c coupling.

E-C couplings is the series of events that link surface membrane depolarization to calcium release from the SR.

EJSR Extended Junctional SR: an extensive SR cistern associated with RyRs bit not with T tubules or plasmalemma.

Corbular SR: a shorter version of EJSR

Junctional gap: the narrow space separating SR AND T tubule,plsmalemma at CRUs.

CEU: calcium entry unit indicates sites involved in SOCE.

Sarcotubular system: it includes SR and T tubules

Resting sarcomere length: the length of sarcomeres in a skeletal muscle that is not restrained by attachment to bone and is not stimulated

Bridge-free region of thick filament: the domain that bears no cross brides. In skeletal muscle it is in the center of the filament.

Skinning: a procedure by which the surface barrier to solutes penetration is interrupted. It can be done mechanically or by chemical means.

Skeletal muscle: All voluntary muscles in vertebrates are skeletal muscles. Invertebrates do not have skeletal muscles, because they do not have skeletons

Free SR, T tubules fSR, $\mathrm{fT}$ : domains of SR and T tubules that are not involved in CRUs. Junctional SR, T tubules, jSR, JT: domains of SR and T tubules that form parts of CRUs $\mathrm{DHPR}=$ dihydropyridine receptor. $\mathrm{A}$ jargon term used in the muscle literature to indicate the CaV1.1 and CaV 1.2 channels involved in excitation contraction coupling of skeleton and cardiac muscles.

TEM: transmission electron microscopy, the standard microscopy for small objects and thin sections.

SEM: scanning electron microscopy. Allows imaging of solid objects

LM light microscopy used to image immunolabeled specimens

E \#: embryonal days, indicates age of mammalian embryo starting at fertilization

PF\#: post fertilization. Indicates days after egg fertilization.

DFM, IFM: direst and indirect fought muscles. See text.

\section{References}

Airey, J.A., Baring, M.D., Beck, C.F., Chelliah, Y., Deerinck, T.J., Ellisman, M.H., Houenou, L.J., McKemy, D.D., Sutko, J.L., and Talvenheimo, J. Failure to Make Normal Ryanodine Re-ceptor is an Early Event Associated With the Crooked 
Neck Dwarf (cn) Mutation in Chicken. Dev. Dyn. 197:169-188, 1993.

Appelt, D., Buenviaje, B., Champ, C. and Franzini-Armstrong, C. Quantitation of feet content in two types of muscle fibers from hind limb of the rat. Tissue and Cell 21:783-794, 1989.

Appelt, D., Shen, V. and Franzini-Armstrong, C. Quantitation of Ca ATPase, feet and mitochondria in super fast muscle fibres from the toadfish, Opsanus tau. J. Muscle Research and Cell Motility. 12: 543-552, 1991.

Bard, F., Franzini-Armstrong, C. and Ip, W. Rigor cross bridges are double headed in fast muscle from crayfish. J. Cell Biol. 105: 2225-2234, 1987.

Bard, F. and Franzini-Armstrong, C. "Extra" actin filaments at the periphery of skeletal muscle myofibrils. Tissue and Cell.23: 191-197, 1991.

Barone, V., Bertocchini, F., Bottinelli, R., Protasi, F., Allen, P.D., Franzini Armstrong, C., Reggiani, C. and Sorrentino, V. Contractile impairment and structural alterations of skeletal muscle from knockout mice lacking type 1 and type3 ryanodine receptors. FEBS Letters 422: 160-164, 1998.

Bischoff, R. and Franzini-Armstrong, C. Satellite and Stem Cells in Muscle Regneration. In Engel, A.G. and FranziniArmstrong, C. Eds. Myology III Edition. McGrawHill NY, pp 66-86, 2004.

Block, B., Leung, A., Campbell, K.P. and Franzini-Armstrong, C. Structural evidence for direct interaction between the molecular components of the transverse tubules/sarcoplasmic reticulum junction in skeletal muscle. J. Cell Biol 107:25872600, 1988.

Boncompagni S, Rossi AE, Micaroni M, Hamilton SL, Dirksen RT, Franzini-Armstrong C and Protasi F. Characterization and temporal development of cores in a mouse model of malignant hyperthermia. Proc Natl Acad Sci U S A. 106: 2199622001, 2009a

Boncompagni, S., Rossi, A.E., Micaroni, M. et al., "Mitochondria are linked to calcium stores in striated muscle by developmentally regulated tethering structures," Molecular Biology of the Cell, vol. 20, no. 3, pp. 1058-1067, 2009b.

Boncompagni S., Loy R.E., Dirksen R.T. and Franzini-Armstrong C. The 14895T mutation in the type 1 ryanodine receptor induces fiber-type specific alterations in skeletal muscle that mimic premature aging. Aging Cell 9: 958-970, 2010.

Boncompagni, S., Thomas, M., Lopez, J.R., Allen, P.D., Yuan, O., Kranias, E.G., Franzini-Armstrong' C. and. Perez, C.F. Triadin/Junctin double null mouse reveals a differential role for triadin and junctin in anchoring CASQ to the jSR and 
regulating $\mathrm{Ca}^{2+}$ homeostasis. PLoS ONE 7(7): e39962. 2012 a.

Boncompagni, S, Protasi, F and Franzini-Armstrong, C. Sequential Stages in the Age dependent Gradual Formation and Accumulation of Tubular Aggregates in Fast Twitch Muscle Fibers: SERCA and Calsequestrin Involvement. Age. 34:27$41,2012 b$.

Boncompagni S, Michelucci A, Pietrangelo L, Dirksen RT, Protasi F. Exercise-dependent formation of new junctions that promote STIM1-Orai1 assembly in skeletal muscle. Sci Rep. 7:14286, 2017.

Bossen EH, Sommer JR Comparative stereology of the lizard and frog myocardium. Tissue Cell 16:173-178, 1984. Brochet, D.X.P., Yang, D., Di Maio, A., Lederer, W.J., Franzini-Armstrong, C. and Cheng, H. Ca ${ }^{2+}$ blinks: rapid nanoscopic store calcium signaling. PNAS 102: 3099-3104, 2005.

Burkeen, A.K., Maday, S. L., Rybicka, K. K., Sulcove, J. A., Ward, J., Huang, M. M., Franzini-Armstrong, C., and Allen, T. StC. Disruption of Caenorhabditis elegans Muscle Structure and Function Caused by Mutation of Troponin I. Biophys. J. 86: 991-1001, 2004.

Campbell, K.R., Franzini-Armstrong, C. and Shamoo, A.E. Further characterization of light and heavy sarcoplasmic reticulum vesicles. Identification of the 'sarcoplasmic reticulum feet' associated with heavy sarcoplasmic reticulum vesicles. Biochem. Biophys. Acta. 602:97-116, 1980.

Cardenas, C., Escobar, M., Härtel, S. and Franzini-Armstrong, C. Visualization of inositol 1,4,5-trisphosphate receptors on the nuclear envelope outer membrane by deep etching electron microscopy. J. Structural Biology. 171: 372-381, 2010.

Castellani, L., Hardwicke, PM.D. and Franzini-Armstrong, C. Effect of Ca2+ on the dimeric structure of scallop sarcoplasmic reticulum. J. Cell Biol. 108: 511-520, 1989.

Castillo de Maruenda, E. and Franzini-Armstrong, C. Satellite and invasive cells in frog sartorius muscle. Tissue and Cell 10:749-772, 1978.

Chopra N, Kannankeril PJ, Yang T, Hlaing T, Holinstat I, Ettensohn K, Pfeifer K, Akin B, Jones LR, Franzini-Armstrong C, Knollmann BC. Modest Reductions of Cardiac Calsequestrin Increase Sarcoplasmic Reticulum Ca2+ Leak Independent of Luminal Ca2+ and Trigger Ventricular Arrhythmias in Mice. Circ Res . 101:617-626, 2007.

Chopra , N., Yang, T., Asghari, P., Moore, E.D., Huke, S., Akin, B.,. Cattolica, R.A., Perez, C.F., Hlaing,T., KnollmannRitschel, B.E.C., Jones, L., Pessah, I.N., Allen, P..D., Franzini-Armstrong, C. and Knollmann, B.C. Ablation of triadin causes structural remodeling of cardiac $\mathrm{Ca}^{2+}$ release units, impaired excitation contraction coupling, myocyte $\mathrm{Ca}^{2+}$ 
overload and ventricular arrhythmias in mice. PNAS, 106:7636-41, 2009.

Close, M., Perni, S., Franzini-Armstrong, C. and Cundall, D. Highly extensible skeletal muscle in snakes. J. Exp. Biol. 217:2445-8 2014.

Crossley, A. C. Ultrastructural changes during transition of larval to adult of intersegmental muscle at metamorphosis in the blowfly Calliphora erythrocephala. I Dedifferentiation and myoblast fusion J Embryol exp Morphol. 27:4374-85, 1972.

Dayal, A., Schredelseker, J., Franzini-Armstrong,,C. and Grabner, M. Skeletal muscle excitation-contraction coupling is independent of a conserved heptad repeat motif in the C-terminus of the DHPR!1a subunit. Cell Calcium 47: 500-506. 2010.

Di Biase, V. and Franzini-Armstrong, C. Evolution of skeletal type e-c coupling: a novel means of controlling calcium delivery. J. Cell Biol. 171:695-704, 2005.

Di Maio, A., Ter Keurs,. H.E., Franzini-Armstrong, C. T-tubule profiles in Purkinje fibres of mammalian myocardium. J Muscle Res Cell Motil . 28: 115-121, 2007.

Dulhunty, A.F. and C. Franzini-Armstrong. The relative contributions of folds and caveolae to the surface membrane of frog skeletal muscle fibre to different sarcomere lengths. J. Physiol. 250:513-539, 1975.

Dulhunty, A.F. and Franzini-Armstrong, C. The passive electrical properties of frog skeletal muscle fibers at different sarcomere lengths. J. Physiol. 266:687-711, 1977.

Eastwood, A.B., Franzini-Armstrong, C. and Peracchia, C. Structure of membranes in crayfish muscle: comparison of phasic and tonic fibers. J. Muscle Research Cell Motility. 3:273-294, 1982.

Escobar M., Cardenas C., Colavita, K., Petrenko N.B. and Franzini-Armstrong C. Structural evidence for perinuclear calcium microdomains in cardiac myocytes. J. Mol. Cell Cardiol 50: 451-459, 2011.

Fang, X., Luo, J., Nishihama, R., Wloka, C., Dravis, C., Travaglia, M., Iwase, M., Vallen, E.A. and Bi, E. Biphasic targeting and cleavage furrow ingression directed by the tail of a myosin II. J Cell Biol. 191:1333-50, 2010.

Felder, E. and Franzini-Armstrong, C. Type 3 ryanodine receptors of skeletal muscle are segregated in a parajunctional position. Procs. Natl. Acad, Sci. USA, 99: 1695-1700. 2002.

Felder, E., Protasi F., Hirsch, R., Franzini-Armstrong, C. and Allen, P.D. Morphology and Molecular Composition of 
Sarcoplasmic Reticulum Surface Junctions in the Absence of DHPR and RyR in Mouse Skeletal Muscle. Biophys. J. 82: 3144-3149, 2002.

Ferguson, D.G. and Franzini-Armstrong, C. The Ca ATPase content of slow and fast twitch fibers of guinea pig. Muscle and Nerve, 11: 561-570, 1988.

Ferguson, D.G., Schwartz, H., and Franzini-Armstrong, C. Subunit structure of junctional feet in triads of skeletal muscle. A freeze-drying, rotary-shadowing study. J. Cell Biology 99: 1735-1742, 1984.

Ferguson, D.G., Franzini-Armstrong, C., Castellani, L., Hardwicke, P.M.D. and Kinney, L.J. Ordered arrays of CaATPase tails on the cytoplasmic surface of isolated sarcoplasmic reticulum. Biophys. J. 48: 597-605, 1985.

Fessenden, J.D., Chen, L., Wang, Y., Paolini, C., Franzini-Armstrong, C., Allen, P.D. Pessah,I.N. Ryanodine Receptor Point Mutant E4032A Reveals A Novel Allosteric Interaction with Ryanodine. Procs. Natl. Acad. Sci. USA 98: 2865-70, 2001.

Flucher, B.E., Takekura, H. and Franzini-Armstrong, C. Development of the excitation-contraction coupling apparatus in skeletal muscle: association of sarcoplasmic reticulum and transverse tubules with myofibrils in developing muscle fibers. Dev. Biol. 160: 135-147, 1993.

Flucher, B.E. and Franzini-Armstrong, C. Formation of junctions involved in excitation-contraction coupling in skeletal and cardiac muscle. Procs. Natl. Acad. Sci. 93:8101-8106, 1996.

Flucher, B.E., Takekura, H. and Franzini-Armstrong, C. Development of the excitation-contraction coupling apparatus in skeletal muscle: association of sarcoplasmic reticulum and transverse tubules with myofibrils in developing muscle fibers. Dev. Biol. 160: 135-147, 1993.

Franzini-Armstrong, C. Fine structures of sarcoplasmic reticulum and transverse tubular system in muscle fibers. Fed. Proc. 23:887-895, 1964.

Franzini-Armstrong, C. Natural variability in the length of thin and thick filaments in single fibers from a crab Portunus depurator. J. Cell Sci. 6:559-592, 1970a.

Franzini-Armstrong, C. Details of the I band structure as revealed by the localization of ferritin. Tissue and Cell 2:327338, 1970b.

Franzini-Armstrong, C. Studies of the triad: I structure of the junction in frog twitch fibers. J. Cell Biol. 47:488-499, 1970c. 
Franzini-Armstrong, C. Studies of the triad: II Penetration of tracers into the junctional gap. J. Cell Biol. 49:196-203, 1971.

Franzini-Armstrong, C. Studies of the triad: III Structure of the junction in fast twitch fiber. Tissue and Cell 4:469- 478, 1972.

Franzini-Armstrong, C. The structure of a simple Z line. J. Cell Biol. 58:630-642, 1973a.

Franzini-Armstrong, C. Studies of the triad: IV Structure of the junction in frog slow fibers. J. Cell Biol. $\underline{56} 1120-128,1973$.

Franzini-Armstrong, C. Studies of the triad: IV Structure of the junction in frog slow fibers. J. Cell Biol. $\underline{56}$ :120-128, I973b.

Franzini-Armstrong, C. Freeze-fracture of striated muscle from a spider. Structural differentiations of sarcoplasmic reticulum and transverse tubular system membranes. J. Cell Biol. 61:50I-513, 1974.

Franzini-Armstrong, C. Freeze-fracture of excitatory and inhibitory synapses in crayfish neuromuscular junctions. J. Microscop. Biol. Cell. 25:217-230, 1976.

Franzini-Armstrong, C. Structure of sarcoplasmic reticulum. Federation Proc. 39:2403-2409, 1980.

Franzini-Armstrong, C. Junctional feet and membrane particles in the triads of a fast twitch muscle fiber. J. Muscle Research Cell Motility. 4:233-252, 1983.

Franzini-Armstrong, C. Freeze-fracture of frog slow tonic fibers. Structure of surface and internal membranes. Tissue and Cell. 16:146-166, 1984.

Franzini-Armstrong, C. Simultaneous maturation of transverse tubules and sarcoplasmic reticulum during muscle differentiation in the mouse. Dev.Biol. 146: 353-363, 1991.

Franzini-Armstrong, C. Annemarie Weber: Ca2+ and the regulation of muscle contraction. Trends Cell Biol. 8:251-253, 1998.

Franzini-Armstrong, C. The sarcoplasmic reticulum and the control of muscle contraction. FASEB J. 13 (suppl.): S266-270, 1999.

Franzini-Armstrong, C. Veratti and beyond: structural contributions to the study of muscle activation. Rend. Fis. Acc. Lincei 13:289-323, 2002.

Franzini-Armstrong, C. The Membrane Systems of Muscle Cells in In Engel, A.G. and Franzini-Armstrong, C. Eds. Myology 
III Edition. McGrawHill NY, pp 232-256, 2004a.

Franzini-Armstrong, C. Functional implications of RyR-DHPR relationships in skeletal and cardiac muscle. Biol. Res. 37: 507-512, 2004b.

Franzini-Armstrong, C. ER-Mitochondria Communication. How Privileged? Physiology (Bethesda) 22:261-8, 2007.

Franzini-Armstrong, C. Architecture and regulation of the $\mathrm{Ca}^{2+}$ delivery system in muscle cells. Appl. Physiol. Nutr. Metab. 34: 3232-327, 2009.

Franzini-Armstrong, C. New and Notable: Can the Arrangement of RyR2 in Cardiac Muscle Be Predicted? Biophys J. 110: 2563-2565, 2016.

Franzini-Armstrong, C. The relationship between form and function throughout the history of excitation-contraction coupling J. Gen Physiol 150 (1): 1-22, 2018.

Franzini-Armstrong, C. and Boncompagni, S The Evolution of the Mitochondria-to-Calcium Release Units Relationship in Vertebrate Skeletal Muscles," J. Biomed. Biotech, vol. 2011, Article ID 8305732011.

Franzini-Armstrong, C. and Di Biase, V. Evolution of skeletal type e-c coupling. Basic Applied Myology, 17: 119-122, 2007.

Franzini-Armstrong, C. and Engel, A.G. Skeletal Muscle: architecture of the membrane system In Muscle. Fundamental Biology and Mechanisms of Diseases. J.A. Hill and E.N. Olson. Academic Press (Elsevier) pp. 763-774, 2012.

Franzini-Armstrong, C. and Ferguson, D.G. Density and disposition of CaATPase in sarcoplasmic reticulum membrane as determined by shadowing techniques. Biophys. J. 48: 607-615, 1985.

Franzini-Armstrong, C. and Kish, C.W. Alternate disposition of tetrads in peripheral couplings of skeletal muscle. J. Muscle Research Cell Motility 16: 319-324, 1995.

Franzini-Armstrong, C. and Nunzi, G. Junctional feet and membrane particles in the triads of a fast twitch muscle fiber. J. Muscle Research Cell Motility. 4:233-252, 1983.

Franzini-Armstrong, C. and Peachey, L.D.. A modified Golgi black reaction method for light and electron microscopy. J. Histochem. Cytochem. 30: 99-105, 1982.

Franzini-Armstrong, C. and Porter, K.R. Sarcolemmal invaginations constituting the T-system in fish muscle J. Cell Biol. 
22:675-696, 1964.

Franzini-Armstrong, C., R. Venosa and P. Horowicz. Morphology and accessibility of the "transverse" tubular system in frog sartorius muscle after glycerol treatment. J. Membr. Biol. 14:197-212, 1973.

Franzini-Armstrong, C., Landmesser, I. and Pilar, G. The size and shape of transverse tubule openings in frog twitch muscle fibers. J. Cell Biol. 64:493-497, 1975

Franzini-Armstrong, C., Eastwood, A.E. and Peachey, L.D. Shape and disposition of clefts, tubules and sarcoplasmic reticulum in long and short sarcomere fibers of crab and crayfish. Cell Tissue Res. 244: 9-19, 1986.

Franzini-Armstrong, C., Kenney, L. and Varriano-Marston, E. The structure of calsequestrin in triads of vertebrate muscle. J. Cell Biol. 105: 49-56, 1987a.

Franzini-Armstrong, C., Gilly, W.F., Aladjem, E. and Appelt, D. Golgi stain identifies three types of fibers in fish muscle. J. Muscle Res. Cell Motility, $\underline{8}:$ 418-427, 1987b.

Franzini-Armstrong, C., Champ, C. and Ferguson, D.G. Discrimination between fast- and slow- twitch fibres of guinea pig skeletal muscle using the relative surface density of junctional transverse tubule membrane. J. Muscle Research and Cell Motility 9: 403-414, 1988a.

Franzini-Armstrong, C., Protasi, F. and Ramesh, V. Comparative ultrastructure of calcium release units in skeletal and cardiac muscle. In Cardiac sarcoplasmic reticulum function and regulation of contractility. R.G. Johnson, R.G. and E.G. Kranias, eds._Ann. NY Acad. Sci. 853:20-31, 1998b.

Franzini-Armstrong, C., Ramesh, V. and Protasi, F. Shapes, sizes and distributions of Ca release units and couplons in a variety of skeletal and cardiac muscles. Biophys J. 77: 1528-1539, 1999.

Gach, M.P., Cherednichenko, G., Haarmann, C., Lopez, J.R., Beam, K.G., Pessah, I.N, Franzini-Armstrong, C. and Allen, P.D. alpha2delta dihydropyridine receptor subunit is a critical element for excitation-coupled calcium entry but not for formation of tetrads in skeletal myotubes. Biophys. J., 94: 3023-3034, 2008.

Glaser, N, lyer VR, Gilly, WF, Franzini-Armstrong C. Functionally driven modulation of sarcomeric structure and membrane systems in the fast muscles of a copepod (Gaussia princeps). Anat. Record 301:2164-2176, 2018.

Hirose, K., Lenart, T.D. , Murray, J., Franzini-Armstrong, C. and Goldman, Y. Flash and Smash: rapid freezing of muscle fibers activated by photolysis of ATP. Biophys. J. 65:397-408, 1993. 
Hirose, K., Franzini-Armstrong, C., Goldman, Y, and Murray, J. Structural changes in muscle cross-bridges accompanying force generation. J. Cell Biol. 127: 763-778, 1994.

Holtzer, H., Hijikata, T., Lin, Z.X., Zhang, Z. Q., Holtzer, S., Protasi, F., Franzini-Armstrong, C. and Sweeney, H.L. Independent assembly of 1.6mm long bipolar MHC filaments and I-Z-I bodies. Cell Structure and Function. 22:83-93, 1997.

Hong, L., Elbl, T., Ward, J., Franzini-Armstrong, C., Rybicka, K.K., Gatewood, B.K., Billie, D.L. and Bucher, E.A. MUP-4 is a novel transmembrane protein with functions in epithelial cell adhesion in Caenorhabditis elegans. J. Cell Biol. 154:403-14, 2001.

Horstick, E.J., Linsley, J.W., Dowling, J.J., McDonal, K.K., Ashley-Koch, A.,. Hauser, M.A., Saint-Amant, L., Satish, A., Cui, W.W., Zhou, W., Sprague, S.M., Franzini-Armstrong, C., Hirata, H. and Kuwada, J.Y. Stac3 is a component of the excitation-contraction coupling machinery and mutated in Native American myopathy. Nat Commun. 4:1952, 2013.

Huang, H., Sun, L.,Ji, S., Zhao, T., Zhang, W., Xu, J., Zhang, J., Wang, Y., Wang, X. Franzini-Armstrong, C., Zheng, M., and Cheng, H. Kissing and nanotunneling mediate intermitochondrial communication in the heart PNAS 110: 2846-2851, 2013.

Jones, L. R., Suzuki, Y. J., Wang, W., Kobayashi, Y. M., Ramesh, V., Franzini-Armstrong, C., Cleemann, L., Morad, M. Regulation of $\mathrm{Ca} 2+$ signaling in transgenic mouse cardiac myocytes overexpressing calsequestrin. J. Clinical Invest. 101:1385-93, 1998.

Kalyanasundaram, A., Bal, N.C., Franzini Armstrong. C., Knollmann, B.C. and Periasamy, M. The calsequestrin mutation CASQ2D307H does not affect protein stability and targeting to the jSR but compromises its dynamic regulation of calcium buffering. J Biol Chem. 285: 3076-3083. 2010 .

Knollmann, B.C., Chopra' N., Hlaing, Th., Budzynski, B., Ettensohn, K., , Horton` K.D., Weissman, N.J., Roden, D.M., Jones' L.R., Franzini-Armstrong' C. and Pfeifer, K. Casq2 Deletion Causes Sarcoplasmic Reticulum Volume Increase, Premature $\mathrm{Ca}^{2+}$ Release and Catecholaminergic Polymorphic Ventricular Tachycardia. J. Clin. Invest. 116:2510-20, 2006.

Lavorato M, Gupta PK, Hopkins PM, Franzini-Armstrong C. Skeletal muscle microalterations in patients carrying Malignant Hyperthermia-related mutations of the e-c coupling machinery. Eur. J. Translational Myology 26: 6105, 2016.

Lavorato, M. and Franzini-Armstrong, C. Practical solutions to frequent problems encountered in thin section electron microscopy. Microscopy Today. May: 1-5, 2017 
Lavorato, M., lyer, V.R., Dewight, W., Cupo, R.R., Debattisti, V., Gomez, L., De la Fuente, S.,, Zhao Y.T., Valdivia, H.H., Hajnóczky, G. and Franzini-Armstrong ,C. Increased mitochondrial nanotunneling activity, induced by calcium imbalance. affects intermitochondrial matrix exchanges. PNAS 114: E849-858, 2017.

Lavorato M, Loro E., Debattisti V., Khurana, T. Franzini-Armstrong C. Elongated Mitochondria Constrictions and fission in muscle fatigue. J Cell Sci. 131(23): 1-8, 2018.

Lavorato, M, lyer, R. Et and Franzini-Armstrong C. A proposed role for non-junctional transverse tubules in s,keletal muscle as flexible segments allowing expansion of the transverse network. Eur. J. Transl. Myol. 29: 86-91, 2019.

Lavorato, M., Formenti, F. and Franzini-Armstrong, C. The structural basis for intermitochondrial communications is fundamentally different in cardiac and skeletal muscle. Exp. Physiol. 105: 606-612, 2020.

Lefebvre JL, Ono F, Puglielli C, Seidner G, Franzini-Armstrong C, Brehm, P and Granato M. Increased neuromuscular activity causes axonal defects and muscular degeneration Development 131:2605-2618, 2004.

Lefebvre JL, Jing L, Beccafico S, Franzini-Armstrong C, Granato M. Differential requirement for MuSK and dystroglycan in generating patterns of neuromuscular innervation. Proc Natl Acad Sci U S A. 104:2483-8, 2007.

Lenart, T.L., Murray, J.M., Franzini-Armstrong, C. and Goldman, Y.E. Structure and periodicities of cross-bridges in relaxation, rigor and during contractions initiated by photolysis of caged Ca2+. Biophys. J. 71:2289-2306, 1996.

Leung, A.., Imagawa, T., Block, B., Franzini-Armstrong, C. and Campbell, K.P. Biochemical and ultrastructural characterization of the 1,4-Dihydropyridine receptor from rabbit skeletal muscle. Evidence for a 52,000 subunit. J. Biol. Chem. 263: 994-1001, 1988.

Linsley JW, Hsub I-Uen, Groomc L, Yarotskyyc V, Lavorato M, Horstick EJ, LinsleyD,Wang W. Franzini-Armstrong C, Dirksen RT, Kuwada JY. Congenital myopathy results from misregulation of a muscle Ca2+ channel by mutant Stac3. PNAS 114: E228-236, 2017.

Loesser, K.E. and Franzini-Armstrong, C. A simple method for freeze-drying of macromolecules and macromolecular complexes. J. Struct. Biol. 103: 48-56, 1990.

Manno C, Figueroa LC, Gillespie D, Fitts R, Kang C, Franzini-Armstrong C, Rios E.

Calsequestrin depolymerizes when calcium is depleted in the sarcoplasmic reticulum of working muscle. PNAS 114: E638-647, 2017. 
Mazanet, R. and Franzini-Armstrong, C. SEM of pericytes in rat red muscle. Microvascular Res. 23:361-369, 1982a.

Mazanet, R., Reese, B.R., Franzini-Armstrong, C., and Reese, T.S. Variability in the structure of satellite cells in normal and injured muscle. Dev. Biol. 93:22-27, 1982b.

Monk, K.R., Voas, M.G., Franzini-Armstrong, C,, Hakkinen, I.S. and Talbot, W.S. Mutation of sec63 in zebrafish causes defects in myelinated axons and liver pathology. Dis Model Mech. 6:135-45, 2013.

McIntosh,B.E. and Ostap, M. Myosin-I molecular motors at a glance.J. Cell Sci. 129: 2689-2695, 2016.

McPherson, P.S., Kim, Y-K., Valdivia, H., Knudson, C.M., Takekura, H., Franzini-Armstrong, C., Coronado, R. and Campbell, K.P. The brain ryanodine receptor: a McPherson, P.S., Kim, Y-K., Valdivia, H., Knudson, C.M., Takekura, H., Franzini-Armstrong, C., Coronado, R. and Campbell, K.P. The brain ryanodine receptor: a caffeine sensitive calcium mrelease channel. Neurone 7: 17-25, 1991.

Moore, E.D., Voigt, T., Kobayashi, Y.M., Isenberg, G., Fay, F.S., Gallitelli, M.F. and Franzini-Armstrong, C. Organization of $\mathrm{Ca}^{2+}$ release units in excitable smooth muscle of the guinea-pig urinary bladder. Biophys. J. 87:1836-1847, 2004.

Mukherjea, M., Llinas, P., Kim, H., Travail, M., Safer, D., Menetrey, J., Franzini-Armstrong, C., Selvin, P.R., Houdusse, A. and Sweeney, H.L. Myosin VI dimerization triggers an unfolding of a three-helix bundle in order to extend its reach. Molecular Cell. 35:305-15, 2009.

Nakai, J., Ogura, T., Protasi, F., Franzini-Armstrong, C. ,Allen, P.D. and Beam, K. G. Functional non- equality of the cardiac and skeletal ryanodine receptors. Procs. Natl. Acad. Sci.94: 1019-1022, 1997.

Northrop, J., Weber, A., Mooseker, M.S., Franzini-Armstrong, C., Bishop, M. F., Dubyak, G. R., Tucker, M. and Walsh, T. P. Different calcium dependence of the capping and cutting activities of villin. J. Biol. Chem.261: 9274-9281, 1986.

Nunzi, M.G. and Franzini-Armstrong, C. The structure of smooth and striated portions of the adductor muscle of the valves in a scallop. J. Ultr. Res. 76: 134-148, 1981.

Page, S.G. Fine structure of tortoise skeletal muscle. J Physiol 197:709-715, 1968

Paolini, C., Fessenden , J.D., Pessah, I.N. and Franzini-Armstrong, C. Evidence for conformational coupling between two calcium channels. Procs. Natl. Acad. Sci. USA 101:12748-12752, 2004a.

Paolini, C., Protasi, F., and Franzini-Armstrong, C. The relative position of RyR feet and DHPR tetrads in skeletal muscle. J. Mol. Biol., 342: 145-153, 2004b. 
Paolini C, Quarta M, Nori A, Boncompagni S, Canato M, Volpe P, Allen PD, Reggiani C and Protasi F. Reorganized stores and impaired calcium handling in skeletal muscle of mice lacking calsequestrin-1 J Physiol 583: 767-784, 2007.

Park, H., Ramamurthy, B. , Travaglia, M., Safer, D., Chen, L-Q., Franzini-Armstrong, C., Selvin, P.R. and Sweeney, H.L. Full-length myosin VI dimerizes and moves processively along actin filaments upon monomer clustering. Molecular Cell 21: 331-336, 2006. Front page

Pellegrino, C. and Franzini, C. An electron microscope study of denervation atrophy in red and white skeletal muscle fibres. J. Cell Biol. 17:327-349, 1963.

Perni, S., Ramesh lyer, V. and Franzini-Armstrong, C. Ultrastructure of cardiac muscle in reptiles and birds: optimizing and/or reducing the probability of transmission between calcium release units. J Muscle Res Cell Motil. 33:145-52, 2012.

Perni S, Close M, Franzini-Armstrong C. Novel details of calsequestrin gel conformation in situ. J Biol Chem. 288(43):31358-62. 2013.

Perni S, Dynes JL, Yeromin AV, Cahalan MD, Franzini-Armstrong C. Nanoscale patterning of STIM1 and Orai1 during store-operated Ca2+ entry. PNAS 112: E5533-E5542, 2015b.

Perni S, Marsden KC, Escobar M, Hollingworth S, Baylor SM, Franzini-Armstrong C Structural and functional properties of ryanodine receptor type 3 in zebrafish tail muscle. J. Gen. Physiol. 145: 173-184, 2015a.

Perni S, Close M, Franzini-Armstrong C Design Principles of Reptilian Muscles: Calcium Cycling Strategies Anat Rec. 299:352-360, 2016.

Perni S, Lavorato M, Beam KG. De novo reconstitution reveals the proteins required for skeletal muscle voltage-induced Ca2+ release. Proc Natl Acad Sci U S A. 114:13822-13827, 2017.

Polyak, E., Standiford, D.M., Yakopson, V., Emerson, C.P. and Franzini-Armstrong, C. Contribution of myosin rod protein to the structural organization of adult and embryonic muscles in Drosophila. J. Mol. Bio.I. 331: 1077-1091, 2003.

Porter, K.R. and Franzini-Armstrong, C. The sarcoplasmic reticulum. Scientific American 212:72-82, 1965.

Protasi, F., Sun, X-H. and Franzini-Armstrong, C. Formation and maturation of calcium release units in developing and adult avian myocardium. Dev. Biol. 173:265-278, 1996. 
Protasi, F., Franzini-Armstrong, C. and Flucher, B. Coordinated incorporation of skeletal muscle dihydropyridine receptors and ryanodine receptors in peripheral couplings of BC3H1 cells. J. Cell Biol.137:859-870, 1997.

Protasi, F., Franzini-Armstrong, C. and Allen, P.D. Role of ryanodine receptors in the assembly of calcium release units in skeletal muscle. J. Cell Biol.140:831-842, 1998.

Protasi, F. Takekura, H., Wang, Y., Chen, S.R.W., Meissner, G. Allen, P.D and Franzini-Armstrong, C. RYR1 and RYR3 have different roles in the assembly of calcium release units of skeletal muscle. Biophys. J. 79 2509-2525, 2000

Protasi, F., Paolini, C., Nakai, J., Beam, K.J., Franzini-Armstrong, C. and Allen, P.D. Multiple regions of RYR1 mediate functional and structural interactions with $\mathrm{a}_{15}$-DHPR in skeletal muscle. Biophys. J. 83: 3230-3244, 2002.

Protasi F., Pietrangelo L. and Boncompagni S. Calcium entry units (CEUs): perspectives in skeletal muscle function and disease J. Muscle Res. Cell Motil. August 2020.

Ralston, E., Ploug, T., Kalhovde, J. and Lømo, T. Golgi Complex, Endoplasmic Reticulum Exit Sites, and Microtubules in Skeletal Muscle Fibers Are Organized by Patterned Activity. J. Neuroscience. 21:875-883, 2001.

Ramesh, V., Sharma, V.K., Shen, S-S. and Franzini-Armstrong, C. Structural proximity of mitochondria to calcium release units in rat ventricular myocardium may suggest a role in calcium sequestration. In Cardiac sarcoplasmic reticulum function and regulation of contractility. R.G. Johnson, R.G. and E.G. Kranias, eds. Ann. NY Acad. Sci. 853: 341-345, 1998.

Rosenberg, A.F., Wolman, M.A., Franzini-Armstrong, C. and Granato M. In vivo nerve-macrophage interactions following peripheral nerve injury. J Neurosci. 32:3898-909, 2012.

Rosenberg AF, Isaacman-Beck J, Franzini-Armstrong C, Granato M. Schwann cells and deleted in colorectal carcinoma direct regenerating motor axons towards their original path. J Neurosci; 34(44):14668-81, 2014.

Sharma, K., Ramesh, V., Franzini-Armstrong, C. and Sheu, S-S. "Transport of Ca2+ from sarcoplasmic reticulum to mitochondria in rat ventricular myocytes," Journal of Bioenergetics and Biomembranes, vol. 32, no. 1, pp. 97-104, 2000.

Shen X, Franzini-Armstrong C, Lopez JR,. Jones LR, Kobayashi YM, Wang Y, Kerrick WGL, Caswell AH, Potter JD, Miller T, Allen PD, Perez CF. Triadins modulate intracellular $\mathrm{Ca}^{2+}$ homeostasis but are not essential for excitation-contraction coupling in skeletal muscle. J. Biol. Chem. 282: 37864-37874, 2007.

Schredelseker J. Dayal A. Schwerte T. Franzini-Armstrong C. and Grabner M. Proper restoration of excitation-contraction coupling in the dihydropyridine receptor beta1-null zebrafish relaxed is an exclusive function of the beta1a subunit. $J$ Biol 
Chem 284:1242-51.2009.

Sleeper, M.M., Henthorn, P.S., Vijayasarathy, C., Dambach, T.M., Bowers,T., Tijskens, P., Armstrong, C. and Lankford, E.B. Characterization of Juvenile Dilated Cardiomyopathy in Portuguese Water Dogs. J. Veterinary Intern. Med. 16: 52-62, 2002.

Sun, X-H., Protasi, F., Takahashi, M, Takeshima, H., Ferguson, D.G. and Franzini-Armstrong, C. Molecular architecture of membranes involved in excitation-contraction coupling of cardiac muscle. J. Cell Biol. 129: 659-673, 1995.

Swift, F., Franzini-Armstrong, C., Enger, U.H., Andersson, K.B., Christensen, G.,. Sejersted, O.M. and Louch, W.E. Extreme sarcoplasmic reticulum volume loss and compensatory T-tubule remodeling following Serca2 PNAS 109: 39974001. 2012.

Syed, S., Snyder, G.E., Franzini-Armstrong, C., Selvin, P.R., and Goldman Y. E., Adaptability of myosin V studied by simultaneous detection of position and orientation. EMBO J. 25:1795-1803, 2006 Front page

Takekura, H., Shuman H. and Franzini-Armstrong, C. Differentiation of membrane systems during development of slow and fast skeletal muscle fibres in chicken. J. Muscle Res. Cell Motility. 14:633-645, 1993.

Takekura, H. Sun, X-H and Franzini-Armstrong, C. Development of the excitation-contraction coupling apparatus in skeletal muscle: peripheral and internal calcium release units are formed sequentially. J. Muscle Res. Cell Motil. 15:102118, 1994a.

Takekura, H., Bennett, L., Tanabe, T., Beam, K.G. Franzini-Armstrong, C. Restoration of junctional tetrads in dysgenic myotubes by dihydropyridine receptor cDNA. Biophys. J. $\underline{67}$ : 793-804, 1994b.

Takekura, H., Nishi, M., Noda, T., Takeshima, H., and Franzini-Armstrong, C. Abnormal junctions between surface membrane and sarcoplasmic reticulum in skeletal muscle with a mutation targeted for the ryanodine receptor. Procs. Natl. Acad. Sci. USA. 92: 3381-3385, 1995a.

Takekura, H., Takeshima, H., Nishimura, S., Imoto, K., Takahashi, M., Tanabe, T., Flockerzi, V., Hofman, F. and FranziniArmstrong, C. Co-expression in $\mathrm{CHO}$ cells of two muscle proteins involved in excitation contraction coupling. J. Muscle Res. Cell Motility 16:465-480, 1995b.

Takekura, H. and Franzini-Armstrong, C. Correct targeting of dihydropyridine receptors and triadin in dyspedic mouse skeletal muscle in vivo. Developmental Dynamics 21:372-380, 1999. 
Takekura, H., Flucher, B. and Franzini-Armstrong, C. Sequential docking, molecular differentiation and positioning of Ttubule/SR junctions in developing mouse skeletal muscle. Dev. Biol. 239: 204-214, 2001.

Takekura, $\mathrm{H}$. and Franzini-Armstrong $\mathrm{C}$. The structure of $\mathrm{Ca}^{2+}$ release units in arthropod body muscle indicates an indirect mechanism for excitation-contraction coupling. Biophys. J. 83, 83, 2742-2753, 2002.

Takekura, H., Paolini, C., Franzini-Armstrong, C., Grabner, M. and Flucher, B.E. Differential contribution of skeletal and cardiac II-III loop sequences to the assembly of DHP-receptor arrays in skeletal muscle. Mol. Biol. Cell 15; 5408-5419, 2004.

Tijskens, P., Meissner, G. and Franzini-Armstrong, C. Location of ryanodine and dihydropyridine receptors in frog myocardium. Biophys. J. 84: 1079-1092, 2003a.

Tijskens, P., Jones, L.R. and Franzini-Armstrong, C. Junctin and calsequestrin overexpression in cardiac muscle: the role of junctin and the synthetic and delivery pathways for the two proteins. J. Mol. Cell. Cardiol. 35: 961-974, 2003b.

Tregear, R., Reedy, M.C., Goldman, Y.E., Taylor, K.E., Winkler, H., Franzini-Armstrong, C., Sasaki, H., Lucaveche, C. and Reedy, M.K. Cross-Bridge Number, Position, and Angle in Target Zones of Cryofixed Isometrically Active Insect Flight Muscle Biophys. J. 86:3009-3019, 2004.

Varriano-Marston, E., Franzini-Armstrong, C. and Haselgrove, J. The structure and disposition of cross bridges in deep etched fish muscle. J. Muscle Research and Cell Motility. ㅁ: 363-386, 1984.

Varriano-Marston, E., Franzini-Armstrong, C. and Haselgrove, J. Structure of the M band. J. EM Technique. 6: 131-141, 1987.

Wu, S., Liu, J., Reedy, M.C., Tregear, R.T., Winkler, H, Franzini-Armstrong, C., Sasaki, H,, Lucaveche, C., Goldman, Y.E., Reedy, M.K. and Taylor, K.A. Electron tomography of cryofixed, isometrically contracting insect flight muscle reveals novel actin-myosin interactions. PLoS One 5: e12643, 2010.

Wu, S., Liu, J., Reedy, M.C., Perz-Edwards, R.J., Tregear, R.T., Winkler, H., Franzini-Armstrong, C., Sasaki, H., Lucaveche, C., Goldman, Y.E., Reedy, M.K. and Taylor, K.A. Structural changes in isometrically contracting insect flight muscle trapped following a mechanical perturbation. PLoS One. 7:e39422. 2012.

Yiping, L., Appelt, D., Kelly, A.M. and Franzini-Armstrong, C. Differences in the histogenesis of EDL and diaphragm in rat. Developmental Dynamics 193:359-369, 1992. 
Yuen, B., Boncompagni, S., Feng, W., Yang, T., Lopez, J.R., Matthaei, K.I., Goth, S.R., Protasi, F., Franzini-Armstrong, C., Allen, and Pessah, I.N. Mice expressing T4826I-RYR1 are viable but exhibit sex- and genotype-dependent susceptibility to malignant hyperthermia and muscle damage. FASEB J. 26: 1311-1322, 2012.

Zhang, L., Franzini-Armstrong, C., Ramesh, V. and Jones, L. Structural alterations in cardiac calcium release units resulting from overexpression of junctin. J. Mol. Cell. Cardiol. 33:233-247, 2001.

Zvaritch E, Depreux F, Kraeva N, Loy RE, Goonasekera SA, Boncompagni S, Kraev A, Gramolini AO; Dirksen RT,

Franzini-Armstrong C, Seidman CE, Seidman JG. MacLennan DH. A Ryr1 ${ }^{14895 T}$ Mutation Abolishes $\mathrm{Ca}^{2+}$ Release Channel Function and Delays Development in Homozygous Offspring of a Mutant Mouse Line. Procs. Natl. Acad. Sci USA. 104: 18537-42, 2007.

\section{Acknowledgements}

I thank my husband, Clay, and son and daughters, John, Katie, Sandra and Cecilia, for allowing me to spend most of my time at the microscope. I am indebted to the Editor (Cecilia Armstrong) for correcting my mistakes and to Gabriele Marinello, co-founder of Qeios, for his generous advice and help in submitting the images. 
